\title{
Force Sensing Insole for a Balance Enhancement System
}

by

Patricia Giacoman Zarzar

\author{
A Thesis submitted to \\ the Faculty of Graduate Studies and Research \\ in partial fulfilment of \\ the requirements for the degree of \\ Master of Applied Science
}

Ottawa-Carleton Institute for

Biomedical Engineering

Department of Mechanical and Aerospace Engineering

Carleton University

Ottawa, Ontario, Canada

September 2014

Copyright (C)

2014 - Patricia Giacoman Zarzar 


\section{Abstract}

Balance impairment is common in the elderly, which may be improved by detecting eminent falls and informing the user. Force and foot localization sensors are necessary to measure the balance as required by such assistive devices.

This thesis presents the design and evaluation of an insole for measurement of the complete ground reaction forces and center of pressure that can be integrated on a balance enhancement system. The insole was prototyped using rubber and a number of small force sensing elements. A calibration procedure was implemented for the sensors to calculate the vertical load and an artificial neural network model was implemented on training data to predict shear loads. Experiments with healthy subjects were conducted to evaluate the performance of the insole on both standing and walking conditions. The results show that the insole is capable of measuring the vertical component of the ground reaction force with good accuracy compared to a force plate, and the neural network was able to produce an estimate of the shear forces. Moreover, the insole is capable of measuring the variations of the center of pressure on different standing conditions and during walking. 
To God, for being always present in my life.

To my parents Manuel Giacoman Murra and Maria Clara Zarzar Charur for teaching me to follow my dreams. Your unconditional love and support helped me become the person I am today.

To my brother Manuel, for his motivation and support to undertake this adventure. To my sister Elizabeth, for being always there to listen. 


\section{Acknowledgments}

I would like to thank my supervisor Mojtaba Ahmadi for his support and guidance throughout this research. I would also like to acknowledge Ayron Catteau for his help with the Raspberry Pi programming and continuous suggestions.

Also, I want to thank Steve Truttmann and Alex Proctor for their assistance in the molding process, and all the students at the ABL Lab for their comments and time shared in the lab. Finally, I would like to acknowledge the financial support of CONACYT, the Secretariat of Public Education (SEP) and the Mexican Government, who made this work possible. 


\section{Table of Contents}

Abstract

Acknowledgments $\quad$ iv

$\begin{array}{ll}\text { Table of Contents } & \text { v }\end{array}$

List of Tables $\quad$ ix

List of Figures $\quad$ xi

List of Acronyms xviii

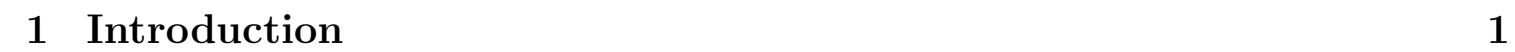

1.1 Motivation . . . . . . . . . . . . . . . . . . . . . . . 3

1.2 Objectives . . . . . . . . . . . . . . . . . 4

1.3 Contributions . . . . . . . . . . . . . . . . . 5

1.4 Outline. . . . . . . . . . . . . . . . . . . 6

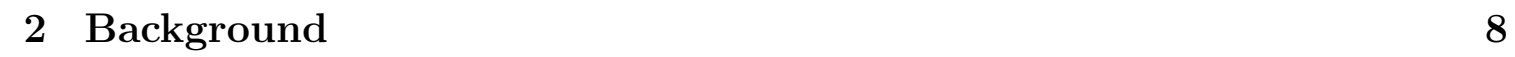

2.1 Assessment of balance . . . . . . . . . . . . . . . . . . . . . . . . . . . 8

2.1 .1 Clinical balance tests . . . . . . . . . . . . . . . 8

2.1 .2 Posturography . . . . . . . . . . . . . . . . . . . . 10

2.2 Gait Fundamentals . . . . . . . . . . . . . . . . . . . . . . . . . . . 11 
2.2 .1 Ground reaction force $\ldots \ldots \ldots \ldots$

2.2 .2 Center of pressure . . . . . . . . . . . . . . . . 12

2.3 Biomechanical models of balance $\ldots \ldots \ldots \ldots \ldots$

$2.4 \quad$ Current technology $\ldots \ldots \ldots \ldots \ldots \ldots$

$2.4 .1 \quad$ Force plates . . . . . . . . . . . . . . . . . . 17

2.4 .2 Pressure sensing systems $\ldots \ldots \ldots \ldots \ldots$

$2.4 .3 \quad$ Motion Capture systems . . . . . . . . . . . . . . . . . . 19

2.4 .4 Low cost mobile systems . . . . . . . . . . . . . . . 20

$2.4 .5 \quad$ Mobile force plates $\ldots \ldots \ldots \ldots \ldots \ldots$

2.5 Tactile feedback $\ldots \ldots \ldots \ldots \ldots \ldots \ldots$

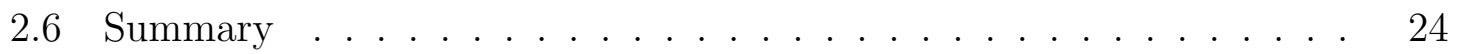

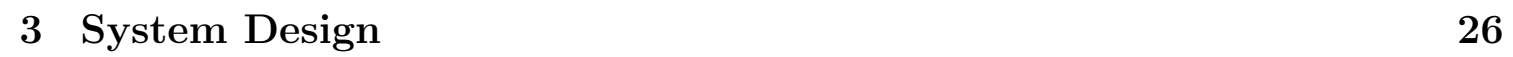

3.1 Design requirements $\ldots \ldots \ldots \ldots \ldots$

3.2 Sensor selection $\ldots \ldots \ldots \ldots$

$3.2 .1 \quad$ Sensor description and signal conditioning $\ldots \ldots \ldots \ldots . . \ldots 32$

3.3 Insole Design $\ldots \ldots \ldots \ldots \ldots \ldots$

3.3 .1 Selection of material . . . . . . . . . . . . . 35

3.3 .2 Distribution of forces under the foot during stance phase . . . 37

3.3 .3 Number of sensors . . . . . . . . . . . . . . . . . . . 42

3.3 .4 Sensor material characterization . . . . . . . . . . . . . . 42

3.3 .5 Simulation and final design $\ldots \ldots \ldots$. . . . . . . . 43

3.3 .6 Implementation . . . . . . . . . . . . . . . . . 50

3.4 Data acquisition system $\ldots \ldots \ldots \ldots \ldots \ldots$

3.5 Summary $\ldots \ldots \ldots \ldots \ldots \ldots \ldots$

\begin{tabular}{|lll}
\hline 4 & Evaluation of force sensing insole & 59
\end{tabular}

4.1 Calibration $\ldots \ldots \ldots \ldots$ 
4.2 First prototype experiments $\ldots \ldots \ldots \ldots \ldots$

$4.2 .1 \quad$ Standing experiments $\ldots \ldots \ldots \ldots \ldots$

$4.2 .2 \quad$ Walking experiments . . . . . . . . . . . . . . . . 69

4.3 Preliminary experiments with final insole $\ldots \ldots \ldots \ldots . \ldots . \ldots 73$

$4.3 .1 \quad$ Standing experiments $\ldots \ldots \ldots \ldots \ldots$

4.3 .2 Walking experiments . . . . . . . . . . . . 76

4.4 Summary $\ldots \ldots \ldots \ldots \ldots \ldots$

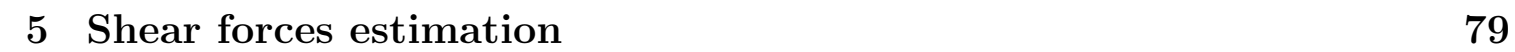

5.1 Previous attempts at estimating shear components of the ground reaction force $\ldots \ldots \ldots \ldots \ldots \ldots \ldots$

5.2 Artificial Neural Networks $\ldots \ldots \ldots$. . . . . . . . . . . . 81

5.2 .1 Training of Neural Networks . . . . . . . . . . . . . 82

$5.3 \quad$ Methodology $\ldots \ldots \ldots \ldots \ldots \ldots$

$5.3 .1 \quad$ Input selection $\ldots \ldots \ldots \ldots \ldots \ldots \ldots$

5.3 .2 Data collection . . . . . . . . . . . . . . . . 84

5.3 .3 Model selection . . . . . . . . . . . . . . . . . 85

5.3 .4 Training and assessment of ANN $\ldots \ldots \ldots \ldots$

5.4 Summary $\ldots \ldots \ldots \ldots \ldots \ldots \ldots \ldots$

\begin{tabular}{|lll}
6 & Experiments with the force sensing insole & 89
\end{tabular}

$6.1 \quad$ Standing experiments $\ldots \ldots \ldots \ldots \ldots$

6.1 .1 Results . . . . . . . . . . . . . . . . . . . . . . . . 93

6.1 .2 Discussion . . . . . . . . . . . . . . . . . . . . . . . . . . . 94

$6.2 \quad$ Walking experiments $\ldots \ldots \ldots \ldots \ldots \ldots$

$6.2 .1 \quad$ Coordinate System transformations . . . . . . . . . . . . 98

6.2 .2 Shear forces estimation . . . . . . . . . . . . . . . 100

6.2 .3 Results . . . . . . . . . . . . . . . . . . . . . . . 101 
6.2 .4 Discussion . . . . . . . . . . . . . . . . . . . . . 101

6.3 Summary . . . . . . . . . . . . . . . . . . . 106

\begin{tabular}{|lll}
\hline 7 & Conclusions and Future Work & 107
\end{tabular}

7.1 Conclusions . . . . . . . . . . . . . . . . . . . . 107

7.2 Future Work . . . . . . . . . . . . . . . . . . . . . . . . . . . . . . . . 109

\begin{tabular}{ll}
\hline List of References & 112
\end{tabular}

\begin{tabular}{|ll|}
\hline Appendix A Ethics Clearance Form & 118
\end{tabular}

\begin{tabular}{|lll}
\hline Appendix B Conditioning circuit diagram & 119
\end{tabular}

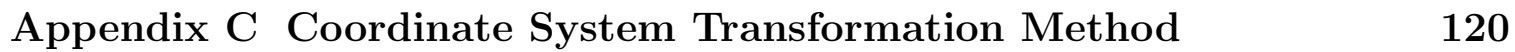

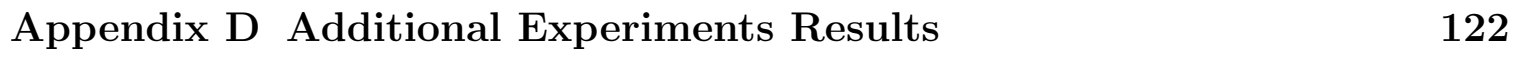




\section{List of Tables}

3.1 Comparison of characteristic of force sensitive resistors. . . . . . . . . 30

$3.2 \quad$ Average force distribution between subjects during push-off phase in gait as percentage of body weight [1]. . . . . . . . . . . . . . . 38

3.3 Maximum force values of men and women in 10 regions of the foot $[2]] \quad$.

3.4 Plantar vertical force distribution values used for simulation as percentage of body weight. . . . . . . . . . . . . . . . . . . . . . . . . 41

3.5 Final dimensions of rubber samples for compressive test. . . . . . . . 43

$3.6 \quad$ Average stress and strain values from the compression test used to characterize material. . . . . . . . . . . . . . . . . . . . . . . . . . . . 45

3.7 Resulting insole displacements from simulation of plantar forces at different percentage of stance. . . . . . . . . . . . . . . . . . . 48

3.8 Position of sensors in the insole frame. . . . . . . . . . . . . . . . . . 51

3.9 Estimated cost per insole. . . . . . . . . . . . . . . . . . . . 57

4.1 Calibration constants for the 14 sensor on both insoles. . . . . . . . . 63

4.2 Correlation and RMS errors of VGRF for prototype 2 of the insole for 10 steps of the walking experiments. . . . . . . . . . . . . . . 71

4.3 Correlation and RMS errors of COP prototype 2 of the insole for 10 steps of the walking experiments. . . . . . . . . . . . . . . . 71

4.4 Correlation and RMS errors for prototype 3 of the insole for the walking experiments . . . . . . . . . . . . . . 76 
4.5 Comparison of correlations (R) and RMSE from the insole prototypes. 78

$5.1 \quad$ Correlation and RMS errors of the estimated shear forces compared with the force plate measurements for different network sizes. . . . . . 87

6.1 Characteristics of participants. . . . . . . . . . . . . . . . 90

6.2 Median and interquartile values of the variation of COP position in the $\mathrm{A} / \mathrm{P}$ and $\mathrm{M} / \mathrm{L}$ direction for the 3 different foot configuration with eyes opened (EO) and eyes closed (EC) for the seven participants. . . 94

6.3 Correlation and RMS errors of VGRF for the participants. . . . . . . 102

6.4 Correlation and RMS errors of COP for the participants. . . . . . . . 102

6.5 Correlation and RMS errors of shear forces for the participants for intra-subject training strategy. . . . . . . . . . . . . . . . . . . . . . . 104

6.6 Correlation and RMS errors of shear forces for the participants for inter-subjects training strategy. . . . . . . . . . . . . . . . . . 104 


\section{List of Figures}

2.1 Typical normal walk cycle showing the different phases and events of gait $[3 \mid . \ldots \ldots \ldots \ldots \ldots \ldots 11$

2.2 The three components of the ground reaction force: vertical (Fz), anterior/posterior $(\mathrm{Fy})$ and medial/lateral $(\mathrm{Fx})$ forces normalized by body weight $[4] . \ldots \ldots \ldots \ldots$. . . . . . . . . . . . . . . 13

$2.3 \quad$ Normal trajectory of the center of pressure of a subject walking bare-

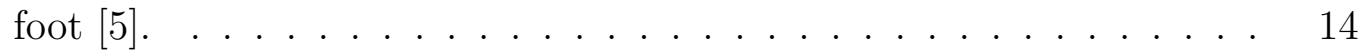

2.4 Recording showing simultaneous COP and COM fluctuations for a sub-

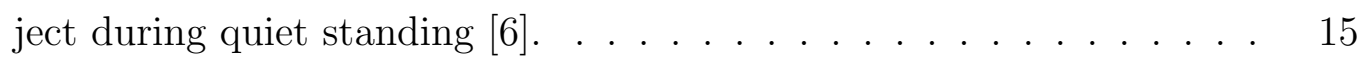

2.5 Vertical ground reaction force on each foot showing the loading and unloading balance mechanism on the medial/lateral direction $[6]$. . . . 16

2.6 Bertec force plate $[7] . \ldots \ldots \ldots \ldots$. . . . . . . . . . . . . 18

2.7 F-scan pressure insole from Tekscan. . . . . . . . . . . . . . . . . . . 18

2.8 Optotrak Certus Motion Capture System. . . . . . . . . . . . . . . . 19

2.9 Components of the Gait shoe system mounted on a shoe [8]. . . . . . 20

2.10 Instrumented insole containing 12 force sensitive resistors for kinetic

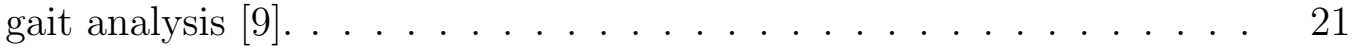


2.11 Prototype of a mobile force plate for measurement of the three components of the ground reaction force. (a) System mounted on a shoe.

(b) Triaxial sensors used on each section. (c) Side view of a section showing the height of the device $[10] . \ldots$. . . . . . . . . . . . 23

3.1 Range of motion of the metatarsal joint of the foot during push off. . $\quad 28$

3.2 Position of sensors on insole compared with plantar pressure distribution. 29

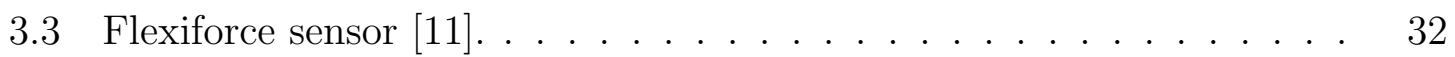

3.4 Recommended circuit for Flexiforce sensor. . . . . . . . . . . . . . . . 33

$3.5 \quad$ Electronic circuit board for conditioning signals from force sensors. . 34

3.6 Design of the insole showing the lines of thiner sections to provide flexibility to the insole and allow the normal flexion of the foot. . . . 35

3.7 Types of rubber hardness and common applications $[12]$. . . . . . . 36

$3.8 \quad$ Force distribution over time under 8 different regions of the foot [13]. 37

3.9 Average forces under the foot versus percent stance for 10 trials of a typical subject $\mid 14] . \ldots \ldots$. . . . . . . . . . . . . . . . . 39

3.10 The 10 different regions of the foot used for the application of the loads for the simulation. . . . . . . . . . . . . . . . . . . . . . . . . . 40

3.11 Rubber sample in MTS machine for compressive test. . . . . . . . . . 44

3.12 Stress-Strain curves for the three rubber samples under uniaxial compression. . . . . . . . . . . . . . . . . . . . . . 45

3.13 Design of first model of the insole showing the position of pucks and thinner sections. . . . . . . . . . . . . . . . . . . . . . . . . . . . . 47

3.14 Displacement simulation results for heel strike and push off times. . . $\quad 49$

3.15 Final insole design showing position of pucks and thinner sections. . . 50

3.16 Coordinate system on the insole. . . . . . . . . . . . . . 51

3.17 Mold used for casting the rubber for the top part of the insole . . . . $\quad 52$ 
3.18 (a) Side view of the final insole showing the total thickness. (b) Enlarged view of the cross section of the insole showing the placement of the sensors between the pucks of the two layers of the insole. . . . . . 53

3.19 Final rubber insole with embedded force sensors. . . . . . . . . . . . . 53

3.20 Components of data acquisition system. . . . . . . . . . . . 54

3.21 Analog to digital converters mounted on Raspberry Pi. . . . . . . . . 55

3.22 Battery pack size comparison with a quarter coin. . . . . . . . . . 56

3.23 User wearing a shoe with the force sensing insole, the Raspberry Pi and the ADC mounted on it. The black case attached to the user ankle contains the electronics for the force sensors. . . . . . . . . . . . . . . 57

4.1 Differences in output voltage of same sensor when loaded with the same mass but with pucks of three different diameters. Active sensor area is $9.5 \mathrm{~mm} . \ldots \ldots \ldots \ldots \ldots$

4.2 Set up for calibration of sensors using calibrated masses. . . . . . . . 61

4.3 Linear and power curve fits for two different sensors. The blue points represents the measured points, the red line is the linear fit, and the blue line is the power function. . . . . . . . . . . . . . . . 62

4.4 Coordinate system on the insole. . . . . . . . . . . . . . . 64

4.5 Static measurement of prototype 1 of the insole compared to a force plate for a subject during single stance. . . . . . . . . . . . . . . . . . 66

$4.6 \quad$ Static measurement of prototype 1 of the insole compared to a force plate for a subject during double stance. . . . . . . . . . . . . . . . . 66

4.7 Static measurement of prototype 1 inside the shoe compared to a force plate for a subject during single stance. . . . . . . . . . . . . . . . . . 67

4.8 Static measurement of prototype 1 inside the shoe compared to a force plate for a subject during double stance. . . . . . . . . . . . . . . . . 67 
4.9 Static measurement of prototype 1 of the insole compared to a force plate for a subject during double stance leaning forth and back showing the decrease of force when the weight is shifted to the back of the foot. 68

4.10 (a) First prototype of the insole. (b) Modified prototype of the insole with the position of one sensor changed from the toes to the heel. . . 69

4.11 Static measurement of prototype 2 of the insole compared to a force \begin{tabular}{|c|c|c|}
\hline plate for a subject during double stance leaning forth and back after \\
\hline
\end{tabular} adding an extra sensor on the heel. . . . . . . . . . . . . . . 70

4.12 Comparison of prototype 2 of the insole (blue) and force plate (red) measurements of GRF and COP for one example step of the left foot during the walking experiments. . . . . . . . . . . . . . 72

4.13 Static measurement of prototype 3 of the insole compared to a force plate for a subject during single stance. . . . . . . . . . . . . . . . . . 74

4.14 Static measurement of prototype 3 of the insole from one leg compared to a force plate for a subject during double stance. . . . . . . . . . . . 74

4.15 Static measurement of prototype 3 of the insole compared to a force

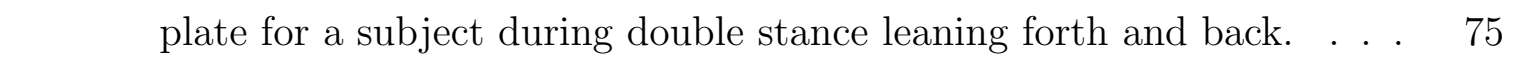

4.16 Comparison of prototype 3 of the insole (blue) and force plate (red) \begin{tabular}{|c|}
\hline measurements of GRF and COP for one example step of the walking \\
\hline
\end{tabular} experiments ........................ 77

$5.1 \quad$ Diagram of a single neuron showing the inputs, weights and bias pa-

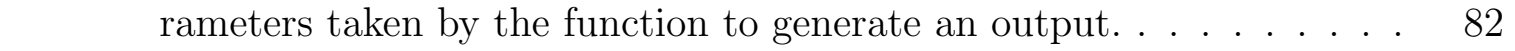

5.2 Neural Network implemented for estimation of shear components of GRF. . . . . . . . . . . . . . . . 85

5.3 Estimated shear forces from the neural network compared with the force plate values. . . . . . . . . . . . . . . . . . . . . . . 87

6.1 Walkway with the two Bertec force plates. . . . . . . . . . . . . . . 90 
6.2 Set up used by participants: (a) Belt with the battery to power the system and wireless transmitter for the Optotrak. (b) Instrumented shoe with Raspberry Pi and electronic box attached to the ankle. . 92

6.3 Comparison of insole (blue stars) and force plate (red stars) measurements of COP from subject 1 standing on one foot with eyes opened (a), (b) and with eyes closed (c), (d). The blue circles represent the position of the sensors on the insole. . . . . . . . . . . . . . . . . 95

6.4 Comparison of insole (blue) and force plate (red) measurements of COP from subject 1 during standing experiments. . . . . . . . . . . . . . . 96

6.5 Top view of the Optotrak, force plate and shoes with their coordinate systems. . . . . . . . . . . . . . . . . . . . . . 98

6.6 Transformation from global coordinate system of the Optotrak and one of the force plates. . . . . . . . . . . . . . . . . . . . . . . . . . . 99

6.7 $\quad$ Transformation from marker coordinate system to one of the insoles. $\quad 99$

6.8 Comparison of insole (blue) and force plate (red) measurements of GRF and COP for one example step of the walking experiments from subject 4. . . . . . . . . . . . . . . . . . . 103

6.9 Estimated shear forces from the intra-subject training strategy of the neural network compared with the force plate values for subject 1. . . 105

A.1 Carleton University Ethics Clearance Form . . . . . . . . . . . . . . . 118

B.1 Schematic diagram of electronic circuit for conditioning signals of force sensors. . . . . . . . . . . . . . . . . . . . . . . . . . . . . . 119

D.1 Comparison of insole (blue) and force plate (red) measurements of COP from subject 2 standing on one foot with eyes opened (a), (b) and with

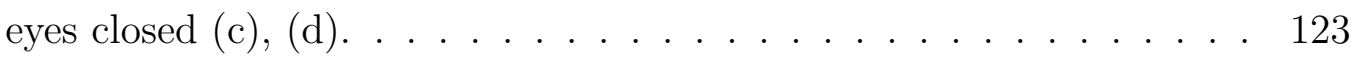


D.2 Comparison of insole (blue) and force plate (red) measurements of COP from subject 3 standing on one foot with eyes opened (a), (b) and with

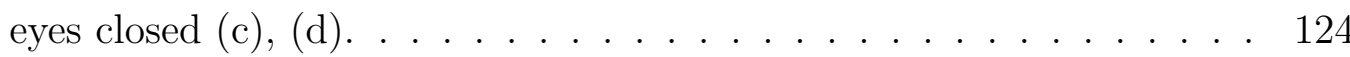

D.3 Comparison of insole (blue) and force plate (red) measurements of COP from subject 4 standing on one foot with eyes opened (a), (b) and with

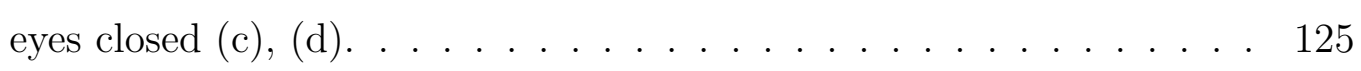

D.4 Comparison of insole (blue) and force plate (red) measurements of COP from subject 5 standing on one foot with eyes opened (a), (b) and with eyes closed $(\mathrm{c}),(\mathrm{d})$.

D.5 Comparison of insole (blue) and force plate (red) measurements of COP from subject 7 standing on one foot with eyes opened (a), (b) and with

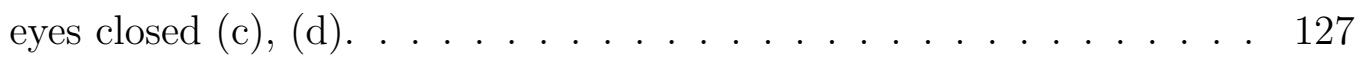

D.6 Comparison of insole (blue) and force plate (red) measurements of GRF and COP for one example step of the walking experiments from subject

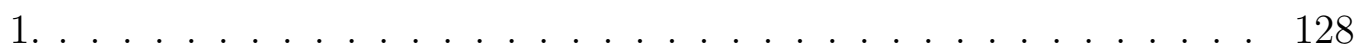

D.7 Comparison of insole (blue) and force plate (red) measurements of GRF and COP for one example step of the walking experiments from subject 2. . . . . . . . . . . . . . . . . . . . . 129

D.8 Comparison of insole (blue) and force plate (red) measurements of GRF and COP for one example step of the walking experiments from subject 3. . . . . . . . . . . . . . . . . . . 130

D.9 Comparison of insole (blue) and force plate (red) measurements of GRF and COP for one example step of the walking experiments from subject 5.

D.10 Comparison of insole (blue) and force plate (red) measurements of GRF and COP for one example step of the walking experiments from subject6. 132 
D.11 Comparison of insole (blue) and force plate (red) measurements of GRF and COP for one example step of the walking experiments from subject7.133 


\section{List of Acronyms}

\begin{tabular}{ll} 
Acronyms & Definition \\
\hline \hline A/P & anterior/posterior \\
BOS & base of support \\
COM & center of mass \\
COP & center of pressure \\
FSR & force sensitive resistor \\
GRF & ground reaction force \\
M/L & medial/lateral \\
RMSE & root mean square error \\
VGRF & vertical ground reaction force \\
\hline
\end{tabular}




\section{Chapter 1}

\section{Introduction}

Standing on two legs, walking, transitioning from one position to another, moving around in the night or with eyes closed are all activities that we perform on a daily basis without giving them much thought, but that require the control of our balance. However, for people with balance disorders, they can become a real challenge and eventually lead to accidents and falls.

The human balance system includes three major sensory systems: the visual, the vestibular and the somatosensory systems 15]. Vision is the primary system used to gather information from the surroundings to plan locomotion and avoid obstacles. The vestibular system is in charge of measuring the linear and angular accelerations of the head. Thus, it provides information about the current position and orientation of the body and its movement. Lastly, the somatosensory system consists of propi-

oreception (the ability to know where our body members are) as well as the sense of touch, particularly under the feet, which provides cues about the direct contact with the floor or any other surface [6].

All these sensory inputs are then integrated by the central neural system (CNS) which generates motor impulses to make postural adjustments and maintain balance.

When one of these sensory inputs is affected, either by injury or disease, a person may experience problems with balance, and the risk of falling increases. Two main 
groups have been identified as at risk: people with vestibular dysfunction and the elderly.

One epidemiological study estimated that $34 \%$ adults aged 40 years or older in the United States (approximately 69 million) have experienced some form of vestibular dysfunction [16]. According to the National Institute on Deafness and Other Communication Disorders (NIDCD), a further 4\% (8 million) of American adults report a chronic problem with balance [17].

Furthermore, it has been estimated that $65 \%$ of individuals older than 60 years of age experience dizziness or loss of balance, often on a daily basis, which may lead to falls [18]. According to the Public Health Agency of Canada more than half of all injuries among Canadians 65 years and over are related to falls [19. Moreover, one third of community-dwelling Canadian seniors experience one fall each year, and half of those will fall more than once. Falls also account for $85 \%$ of seniors injuryrelated hospitalizations and the annual cost to the health system is approximately $\$ 2$ billion [19].

In addition, fear of falling may negatively impact postural control and limit their activities. Yet, by doing so, they are likely to lose strength and flexibility and increase the risk of falling, closing a vicious loop [19].

According to these statistics, the need for a balance enhancement device is apparent. Such a device would improve the quality of life of people with balance problems because it will give them the confidence to perform their daily activities and eventually prevent a fall. The work described in this thesis is part of the BalanceAid Project, which aims to develop a device for ambulatory assessment of balance and tactile feedback to improve the balance of the user. 


\subsection{Motivation}

Sensory substitution has been shown to be a viable solution to provide information from sensory systems that have been affected either by disease or injury [20]. The brain plasticity (the ability of the central neural system to create new connections) allows to substitute one sensory route with a different one.

Vibrotactile elements and inertial sensors to measure body tilt have been previously used in a balance prosthesis $[21,26]$. These studies showed the feasibility to learn and interpret tactile feedback signals to improve postural control in different situations.

Therefore, the motivation of the BalanceAid project is to be able to provide cues to people with balance problems by compensating for their lack of sensory capabilities, so that they can perform their daily life activities without the fear of falling. However, to provide this information, first good quantitative measures of balance are required.

One of the most common techniques for balance assessment is to quantify postural sway during stance by measuring the displacement of the center of pressure from a force plate. The increase on postural sway has been associated with aging, neuropathy, Parkinsons disease, vestibular loss and stroke [27 29]. The center of pressure and related parameters, such as mean velocity and standard deviation of the vertical and horizontal ground reaction forces, have been widely used as a measure for balance assessment in different studies [27, 30 33]. While the center of pressure is a good measure of standing balance, for dynamic situations other parameters such as the shear forces are also required for stability analysis [34].

While force plates remain the gold standard for measuring ground reaction forces and center of pressure, they have the limitation of being fixed in a laboratory or clinical setting. Therefore, to obtain ambulatory measurements of balance mobile solutions are needed. 
Low cost systems containing force sensitive resistors have been previously used on insole applications for measurement of forces under the foot [8,9,35,37. However, these systems are limited to a partial measurement of the vertical ground reaction force, which limits the accuracy and generalization capacities of such devices.

On the other hand, external outsoles have been attached to instrumented shoes for measurement of the complete ground reaction forces on a mobile system $[10,38,39$. These devices consist of two parts placed on the forefoot and heel of the shoes. However, the lack of curvature and flexibility around the forefoot makes it difficult to perform natural walking and not practical for long term use. Moreover, their added height and rigidity also changes the original interface between the shoes and the ground and probably alter normal gait patterns.

To address some of the previous limitations a force sensing insole for measurement of ground reaction forces and center of pressure is presented in this thesis as part of the BalanceAid project.

\subsection{Objectives}

The BalanceAid project being developed at the Advanced Biomechatronics and Locomotion (ABL) laboratory was conceived with the idea of developing a balance enhancement system capable of assessing the stability of the user and preventing a fall. Instead of replacing any specific sensory system (vestibular, visual or somatosensory systems), the device uses the center of pressure as a measure for stability and provides additionally sensory information to the user by means of vibrotactile feedback. In order to have a measurement of the center of pressure both a measurement of the ground reaction forces under the feet and the location and orientation of the feet are necessary.

The BalanceAid project consist of the following elements: 
1. An insole for measuring the ground reaction forces and center of pressure under each foot.

2. A system for measuring the relative location and orientation of the feet.

3. A vibrotactile belt containing the vibrotactile actuators, or tactors, for providing information about the state of balance in real time to the user.

This thesis then focuses on the first element concerning the measurement of the ground reaction forces and center of pressure. The device developed is meant also to be integrated with the foot localization system developed by Ayron Catteau [40], which together can be used to provide the required tactile feedback to the user. Thereupon the person will eventually learn to recognize and use the signals from the tactile feedback system to improve his or her balance and increase confidence while performing daily life activities. The particular objectives of this research are summarized as follows:

1. Develop a low cost insole that fits comfortably inside the shoe and also provides good measurements of the ground reaction force and center of pressure.

2. Evaluate the measurements from the insole through experiments with healthy subjects.

3. Implement technology suitable for integration with the foot localization system for the BalanceAid project.

\subsection{Contributions}

This thesis work presents the following research contributions: 
1. Design and prototyping of a force sensing insole for measurement of ground reaction forces and center of pressure using molded rubber and existing thin force sensing elements.

2. Implementation of the real-time data acquisition and processing software on an embedded processor as required for the integration with the BalanceAid device.

3. Preliminary testing and calibration of the sensors for measurement of normal loads.

4. Implementation and validation of an artificial neural network estimator for the shear components of the ground reaction force during walking.

5. Experimental evaluation of the insole through static and dynamic experiments with seven healthy subjects and analysis of the results.

\subsection{Outline}

This section provides an overview of the remaining chapters of this thesis.

\section{Chapter 2 Background}

This chapter presents a review of the current methods and technology used to measure

and assess the balance and stability of a person. It also presents previous attempts to create mobile and affordable technology for the measurement of the ground reaction force and center of pressure.

\section{Chapter 3 System Design}

This chapter describes the overall design of the force sensing insole. It starts with the general design requirements for the insole, continues with the selection of the sensors and other components of the system and lastly explains the implementation process. 


\section{Chapter 4 Evaluation of force sensing insole}

This chapter presents the calibration process of the force sensors used on the insole and preliminary experiments performed with the first prototype that lead to improvements for the final design. Additionally, some preliminary experiments with the final insole are also presented.

\section{Chapter 5 Shear Forces Estimation}

This chapter describes the estimation of shear components of the ground reaction force by use of artificial neural networks and the experimental results obtained.

\section{Chapter 6 Experiments with the force sensing insole}

This chapter presents the experiments performed with healthy subjects on both standing and walking conditions for the evaluation of the insole. Two training strategies were investigated for the estimation of the shear forces, intra-subject and inter-subject strategies.

\section{Chapter 7 Conclusions and Future Work}

This chapter present the conclusions and recommendations, as well as the future work on this project. 


\section{Chapter 2}

\section{Background}

This chapter presents a review of the literature related to the methods and technology used to measure and assess the balance and stability of a person, as well as the state of the art on mobile technology for the measurement of ground reaction forces and center of pressure.

\subsection{Assessment of balance}

Balance can be defined as the dynamics of the human body to maintain a posture and prevent falling [6]. Currently there are several methods to assess the balance of a person and identify subjects at risk of falling. They go from functional assessment techniques and general physiological check ups, to posturography using force plates and other technologies.

\subsubsection{Clinical balance tests}

To assess the risk of falls and identify balance disorders several clinical tests have been used over the years. These functional balance tests usually use a number scale to rate the performance on a set of motor tasks, or they measure the time a person can maintain a posture. Some examples are: 
Berg Functional Balance Scale. Clinicians rate 14 activities including sitting, standing, and postural transitions from a 0 to 4 point scale. A score less than 45 is associated with increased risk of falling [41].

Activities-Specific Balance Confidence Scale. It is composed of a questionnaire on how the person perceived their own balance while performing 16 daily life activities. However it had not been related directly to falls, but to activities people avoid 42$]$.

Tinetti Balance and Gait Assessment. Also a 14 item test, it is widely used with older people for its sensitivity to identify fallers and its inclusion of both balance and gait evaluation tasks [43].

Get Up and Go Test. A stop-watch is used to measure the duration of functional tasks performed at a comfortable rate, from sitting in a chair, stand up, walk $3 \mathrm{~m}$, turn around, walk back and sit down. This is the shortest and simplest balance test and it only takes 3 minutes [44].

Functional Reach Test. This test assesses limits of standing stability by measuring the maximal distance a person can reach beyond the length of their arm while maintaining a fixed base of support [45].

One Leg Standing Test. Participants are asked to stand on one leg with their arms on the hips, and time is measured from the instant when the foot is flexed off the floor to when it touches the ground or an arm leaves the hip [46].

The advantage of these type of tests is the small amount of time required to be performed, as well as that there is no need for additional technology or devices. However, they do not provide information regarding the cause of balance problems.

To evaluate the underlaying causes of balance deficits, system assessment tests can be performed. One example is the Physiological Profile Approach (PPA) which 
consists of simple clinical tests of vision, cutaneous sensation of the feet, leg muscle force, step reaction time and postural sway.

Although the functional assessment tests can give an indication of the ability of a person to maintain balance and perform different tasks, the results are still prone to subjective evaluation from the person giving the scores. More quantitative measures are then required to assess balance.

\subsubsection{Posturography}

Posturography is the quantitative assessment of postural sway during stance. The evaluation can be either static or dynamic. Static posturography aims to quantify postural sway while a subject stands as still as possible by measuring the displacement of the center of pressure from a force plate. The increase on postural sway has been associated with aging, neuropathy, Parkinsons disease, vestibular loss and stroke [27-29].

Several challenges can be added to the evaluation to investigate different deficits such as reducing the base of support, closing the eyes, adding a softer surface to decrease propioreceptive feedback, or adding a secondary task.

In the case of dynamic posturagraphy, external balance perturbations are introduced such as a moving platform or moving visual surrounding to examine how each sensory system contributes to balance control [46].

The techniques described above are used mainly to identify people with balance problems or at risk of falling. The next sections describe some fundamental gait concepts and introduces human models of balance that provide an insight on the mechanisms used by the body to maintain posture and provide quantitative measures that can be used to assess stability in dynamic situations. 


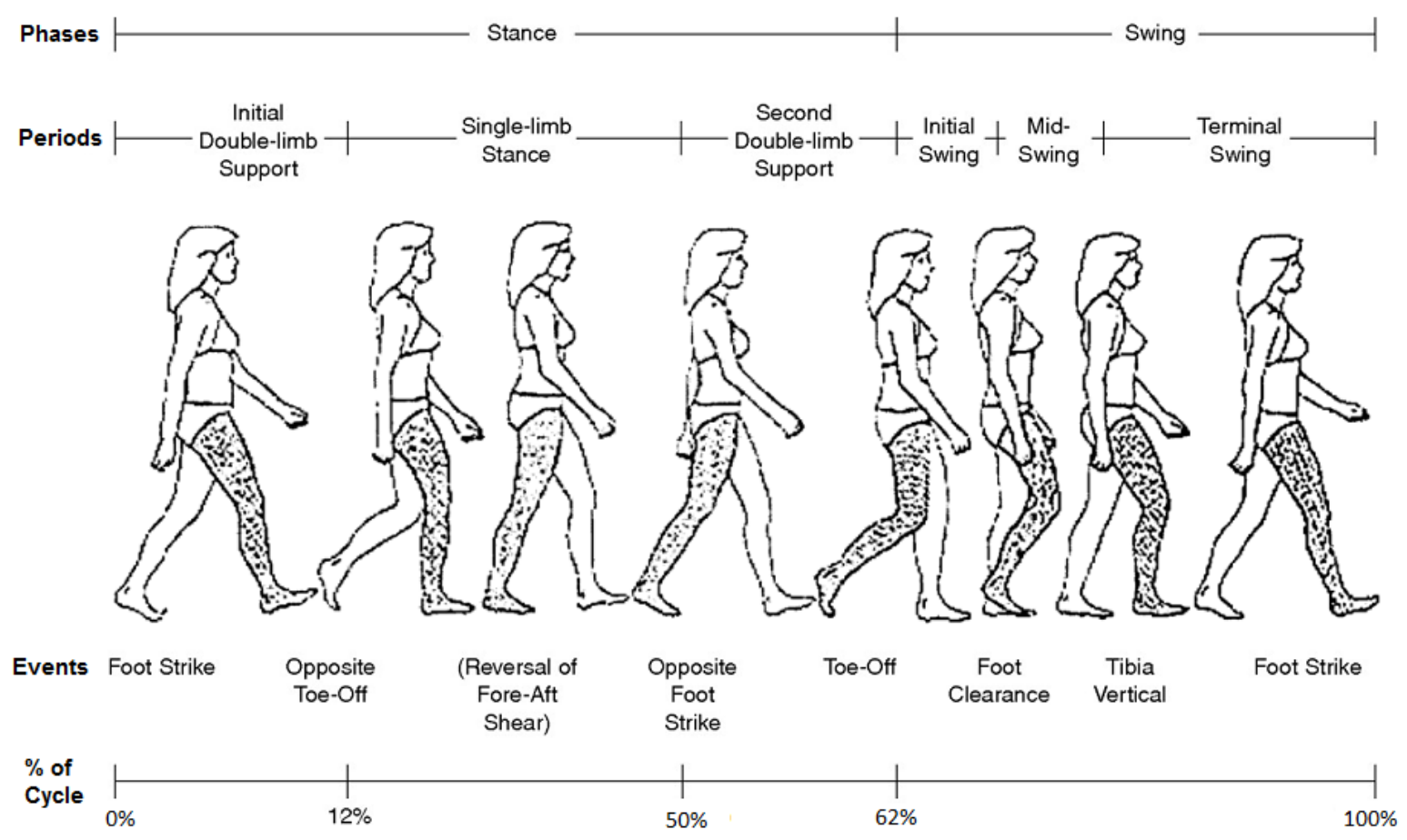

Figure 2.1: Typical normal walk cycle showing the different phases and events of gait 3$]$.

\section{$2.2 \quad$ Gait Fundamentals}

Gait is a term used to refer to the movements of human limbs during locomotion. As shown on Figure 2.1 the gait cycle starts with the first contact of one foot and ends at the next contact of the same foot. The cycle can be divided into two phases: stance and swing phases. The stance phase occurs when the foot is in contact with the ground. It start at heel touchdown and ends at toe-off for the same foot, which in normal walking occurs at about $60 \%$ of the walking cycle. The swing phase occurs when the foot is moving in the air and represents $40 \%$ of the walking cycle. Because each stance phase is $60 \%$, there is a time where both feet are on the ground. This period is called double support and occupies about $10 \%$ of the cycle. Right after toeoff however, there is a period of single support when just one foot is on the ground. 


\subsubsection{Ground reaction force}

During the stance phase of one foot, the total force applied by the foot to the ground is called ground reaction force (GRF). The ground reaction force has three components: the vertical or normal force, the fore-after or anterior/posterior force, and medial/lateral force. As shown in Figure 2.2 the vertical component of the force presents a double hump, which is due to the acceleration and deceleration of the body center of mass through the stance phase. The fore-aft force component shape represents the friction at heel strike and propulsion at push off. Finally the medial/lateral force is small and is directed medially. The resultant of the fore-aft and medial/lateral components is called the shear forces.

\subsubsection{Center of pressure}

The ground reaction force can be used to calculate the center of pressure $(\mathrm{COP})$ of the foot. The COP is the application point of the total ground reaction force vector. At initial contact, the COP is located at the lateral side of the heel and it then travels along the foot until it reaches the metatarsal heads and finally the hallux at toe-off. An example of the COP path is shown in Figure 2.3

\subsection{Biomechanical models of balance}

The mechanisms used by our body to control our balance have been largely studied. Biomechanical models and analyses have now been developed to understand how the CNS controls balance and accomplishes the body synergies. The best known model used to study balance is the inverted pendulum model. In this model we assume that the body is an inverted pendulum pivoting about the ankle. 

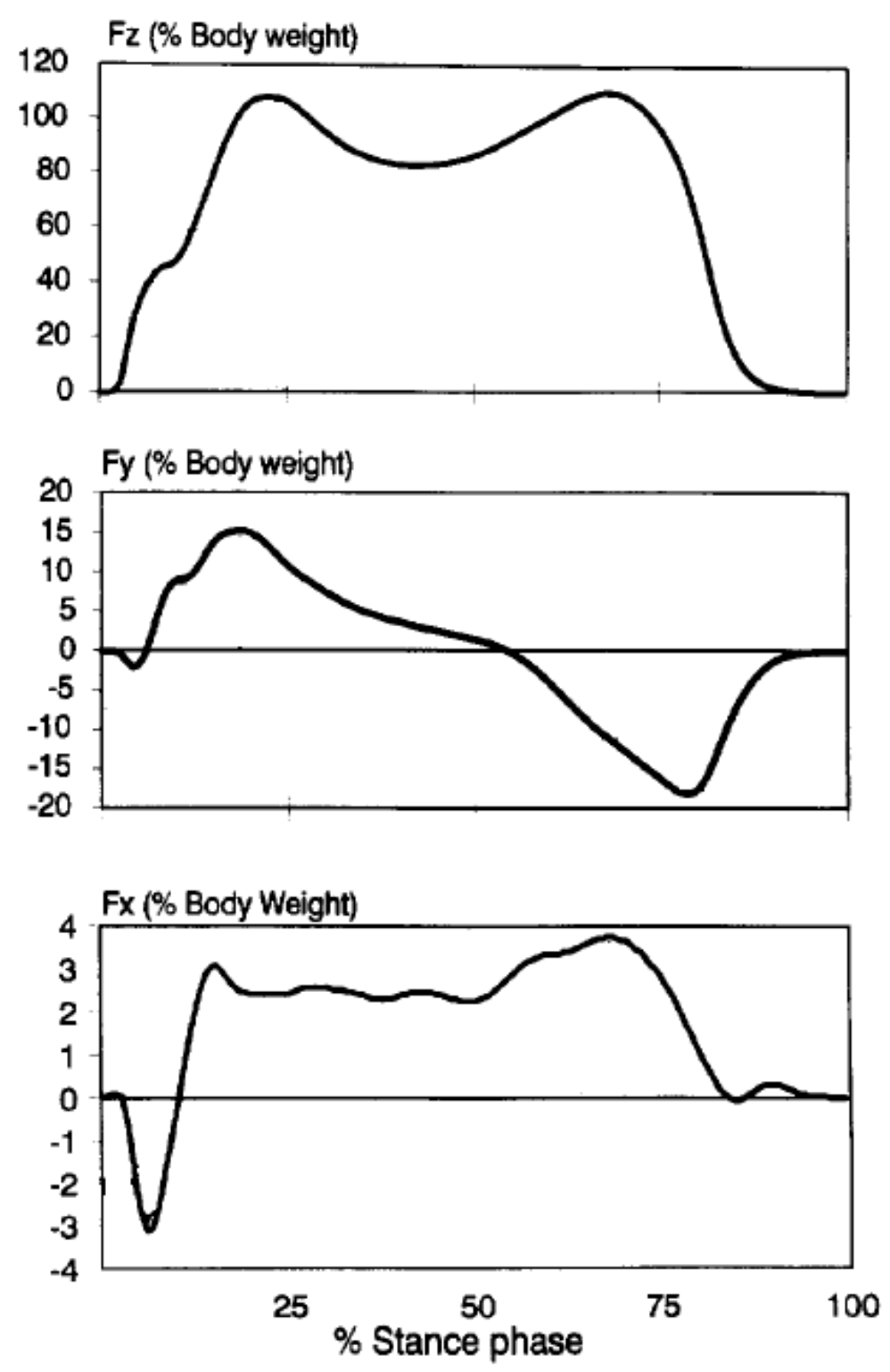

Figure 2.2: The three components of the ground reaction force: vertical $(\mathrm{Fz})$, anterior/posterior (Fy) and medial/lateral $(\mathrm{Fx})$ forces normalized by body weight [4]. 


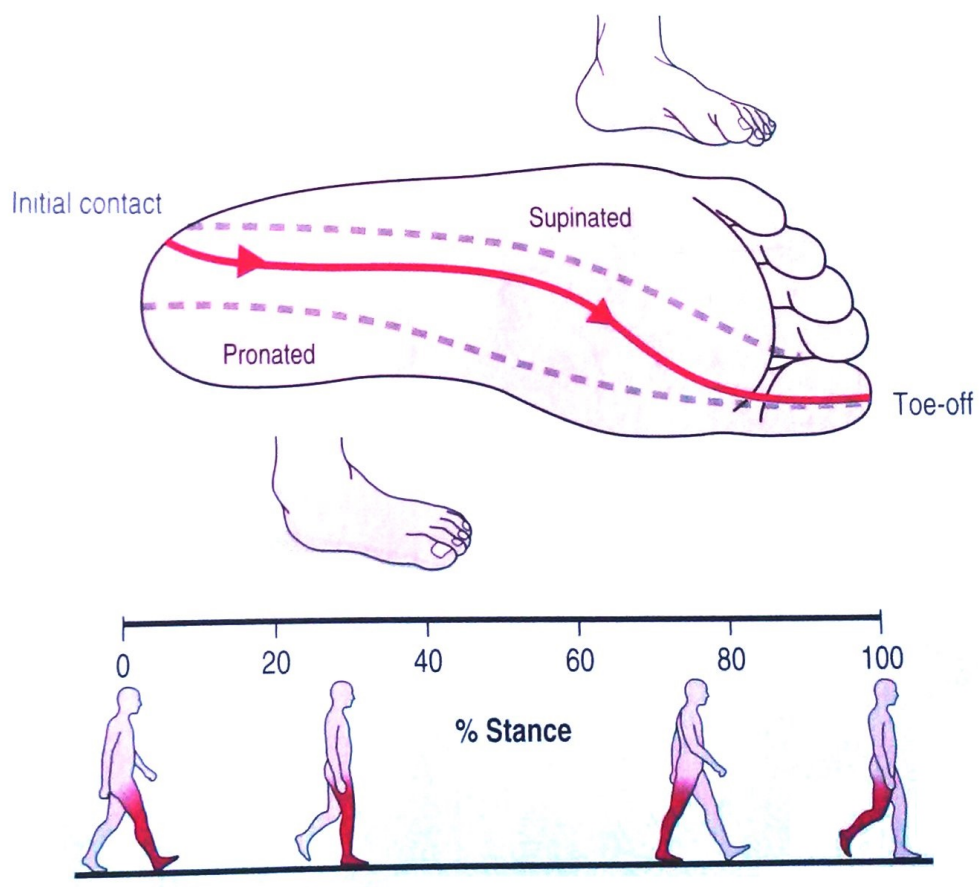

Figure 2.3: Normal trajectory of the center of pressure of a subject walking barefoot $[5]$.

When a person is standing, the body is swaying back and forth. As the center of mass $(\mathrm{COM})$ of the person moves ahead of the center of pressure $(\mathrm{COP})$, an angular acceleration is generated. To correct this sway, the person will shift his COP ahead until it passes the COM and creates a reversing acceleration, eventually returning to the original position. The same holds true for the opposite direction.

If we record both the COP and COM, we will notice that the COP follows the COM but has a greater range of motion (higher frequency and greater amplitude) as shown in Figure 2.4.

This analysis applies in the anterior posterior direction and is called the ankle strategy. A more general model takes also into account the medial/lateral direction. Then, when the two feet are in the ground, the net COP can be calculated as [6]: 


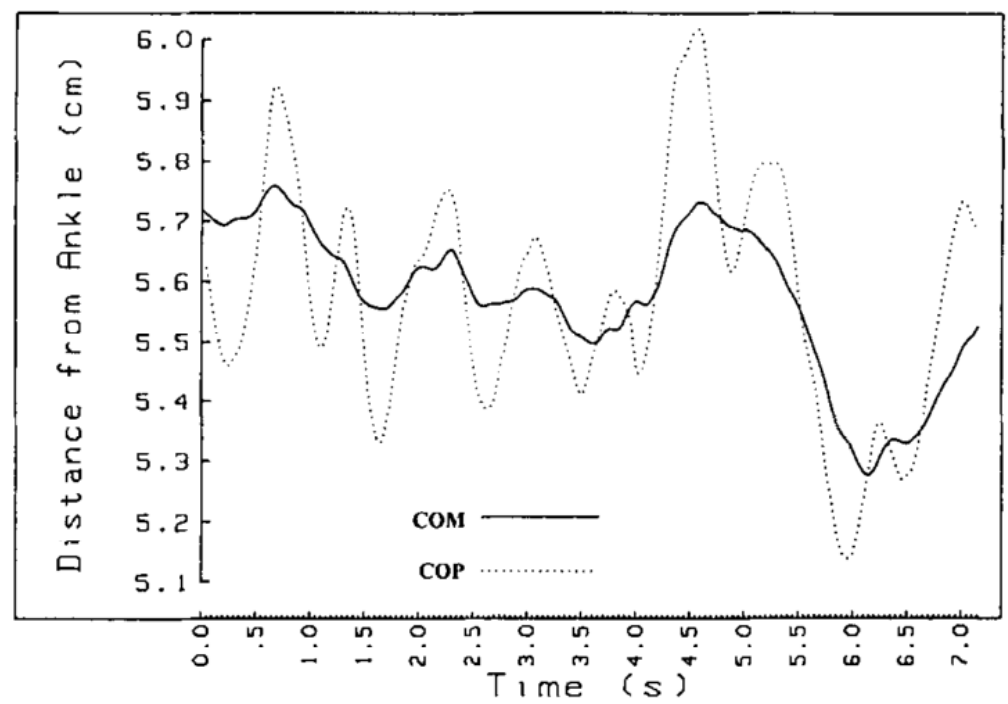

Figure 2.4: Recording showing simultaneous COP and COM fluctuations for a subject during quiet standing [6].

$$
C O P_{n e t}=C O P_{l} \frac{R_{v l}}{R_{v l}+R_{v r}}+C O P_{r} \frac{R_{v r}}{R_{v l}+R_{v r}}
$$

where $C O P_{l}$ and $C O P_{r}$ are the COP's under left and right feet respectively. $R_{v l}$ and $R_{v r}$ are the vertical ground reaction forces under left and right foot respectively.

If we plot the $R_{v l}$ and $R_{v r}$ (Figure 2.5) we will see that they are out of phase, which means that one limb unloads as the other loads. This is known as the hip strategy.

Traditionally, and following the inverted pendulum model, stability was defined as the ability to maintain the body's center of mass over its base of support (BOS) (contact area of the feet). However calculation of the COM requires the use of a motion capture system to reconstruct a kinematic and mass distribution model of the human body, which limits the implementation of the stability models to a laboratory or clinical environment. Since we want to develop a mobile system, we require parameters that can be measured on an ambulatory system. The COP, which is closely 


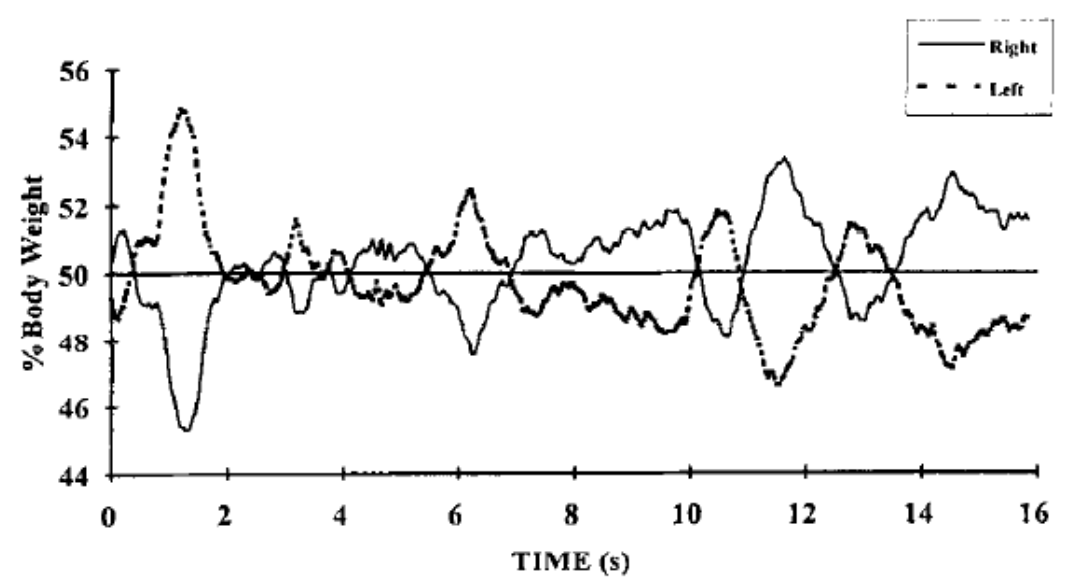

Figure 2.5: Vertical ground reaction force on each foot showing the loading and unloading balance mechanism on the medial/lateral direction [6].

related to the movement of $\mathrm{COM}$, has been widely used for balance assessment in different studies [27].

Parameters such as mean velocity of the COP [30], standard deviation of the COP, the vertical and the horizontal GRF [31], maximal range of COP displacement along the $x$ and $y$ coordinates and sum of the maximal deviations of the COP from its center multiplied by their duration [32] have been used for assessment of balance.

Particularly Popovic et al. [33] came up with an stability criterion to assess balance based on 4 different stability zones: (1)The high preference zone, where the COP resides $99 \%$ of the time; (2)The low preference zone, where the COP resides the next $1 \%$ of the time; (3)The undesirable zone, where the person is forced to change posture to maintain balance; and (4)the unstable zone, where the persons needs to make a step to recover balance. These zones were calculated on experimental trials by asking the participants to stand at different conditions and then slowly pushing them at the shoulder until taking a step was necessary to recover balance.

Finally, research concerning biped robots has also taken concepts and measures from biomechanics for the planning of trajectories, locomotion and stability analysis. 
Particularly, the centroidal moment pivot (CMP) has been used as a measure of stability [34]. In this case, a measurement of the shear forces is required for its calculation.

\subsection{Current technology}

The current gold standard to assess balance and for gait analysis is composed of force plates and motion capture systems. These systems are employed for the evaluation of different pathologies and patient populations. They can be found either in research laboratories or in clinical facilities.

\subsubsection{Force plates}

A force plate is a device that measures the ground reaction force as well as the moments in three dimensions. This information is frequently used to calculate the center of pressure (the application point of the total ground reaction force vector), or is used, together with the kinematic information from motion capture systems, to perform the inverse dynamics needed for gait analysis. In inverse dynamics analysis, the ground reaction and body motion trajectories are used to estimate all human joint moments (or muscle efforts through more advanced models). Force plates have been widely used to assess balance by monitoring the changes on the center of pressure as explained on Section 2.1.2. Examples of suppliers are AMTI, Kistler and Bertec (Figure 2.6). A drawback of the force plate is the inability to calculate the center of pressure on each foot when a person is standing with both feet on a single force plate. Furthermore, the pressure distribution under the feet is not uniform and this information cannot be obtained solely from a force plate, which only provides the resultant forces and moments. 


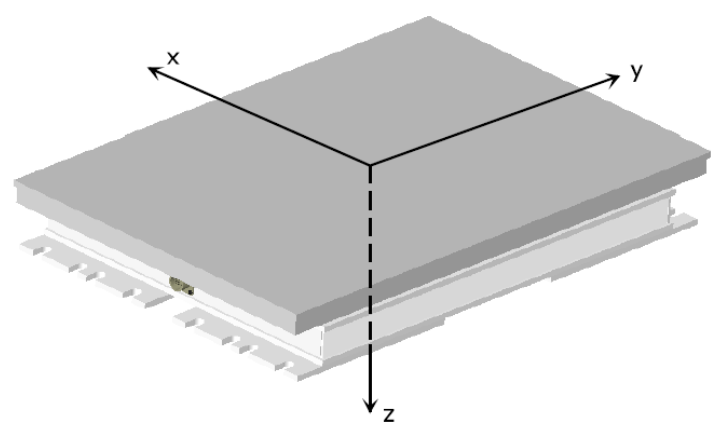

Figure 2.6: Bertec force plate [7].

\subsubsection{Pressure sensing systems}

Another important gait data is the foot pressure distribution. A pressure sensing system consists of multiple arrays of pressure sensing cells constructed on an insole that is placed inside the shoe. They can provide detailed information about the distribution of forces under the foot and have the advantage of being mobile compared to stationary force plates. However they are not capable of measuring the shear components of the ground reaction force. Applications of these systems consist primarily of the investigation of regions of high pressure to diagnose peripheral neuropathy and diabetis, as well as applications in sports and footwear design. Some examples are the Parotec, Pedar and F-scan systems (Figure 2.7).

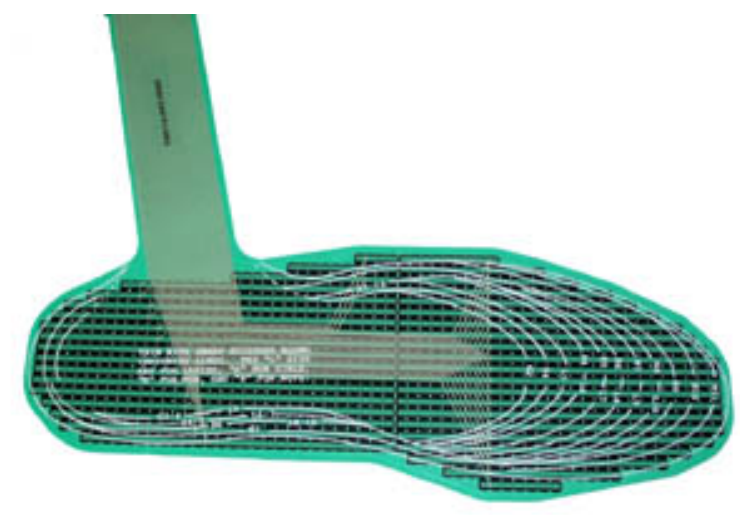

Figure 2.7: F-scan pressure insole from Tekscan. 
Although the pressure sensing insoles have the advantage of being mobile and usable on a wider range of activities and places compared to force plates, they are still too expensive and not able to provide the shear components of the ground reaction force, preventing their used on a real-time balance system.

\subsubsection{Motion Capture systems}

The objective of these systems is to track the motion of body parts. Together with the kinetic information from the force plate, the human model can be reconstructed during walking, and the forces and moments experienced on the joints calculated. Such system consists of a set of cameras fixed in the room and a set of markers placed on the limbs. The correct placement of the markers on the body is important to be able to reconstruct a good model from the motion data. Some common examples are the Vicon system and Optotrak (Figure 2.8).

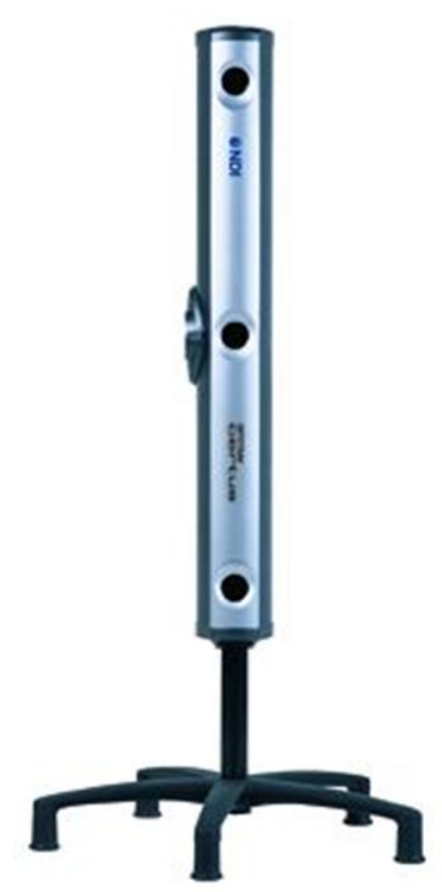

Figure 2.8: Optotrak Certus Motion Capture System. 


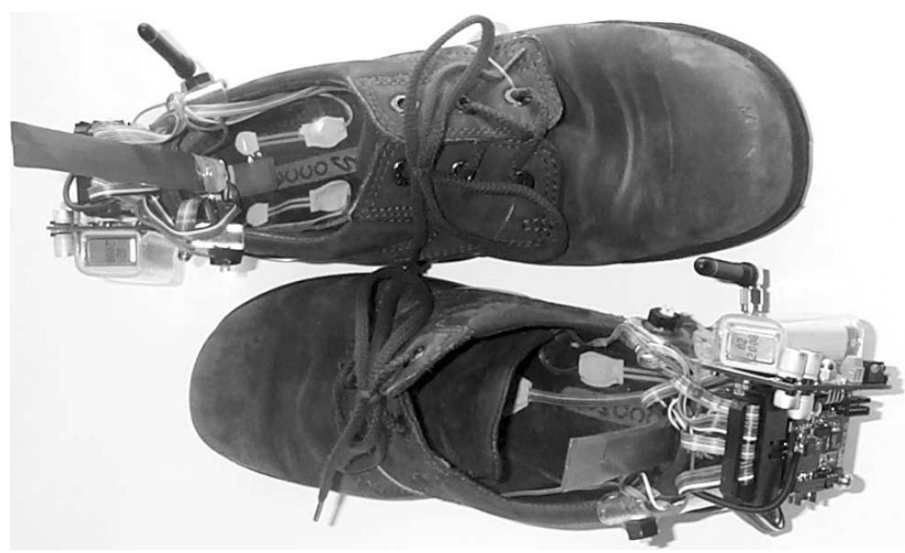

Figure 2.9: Components of the Gait shoe system mounted on a shoe [8].

\subsubsection{Low cost mobile systems}

Several low-cost mobile systems have been proposed to measure ground reaction force for different applications such as gait analysis.

One of these systems frequently used in literature is the Gaitshoe developed by Bamberg et al [8]. An image of the system is shown in Figure 2.9. The prototype consists of an instrumented shoe with force sensitive resistors, bidirectional bend sensors, accelerometers and gyroscopes, among others. The system provides information on the foot pressure, as well as the orientation and position of the feet. This system is able to collect data in different environments without interfering with gait and for long periods of time.

Similar to the previous system, other research has been conducted at the Univeristy of Utah, where the same commercial force sensitive resistors have been used to build instrumented insoles for different applications, from detection of slip by analyzing the acceleration of the foot and the normal force during foot contact with the ground [36] to evaluation of changes on force distribution on the feet during standing for balance assessment 35 


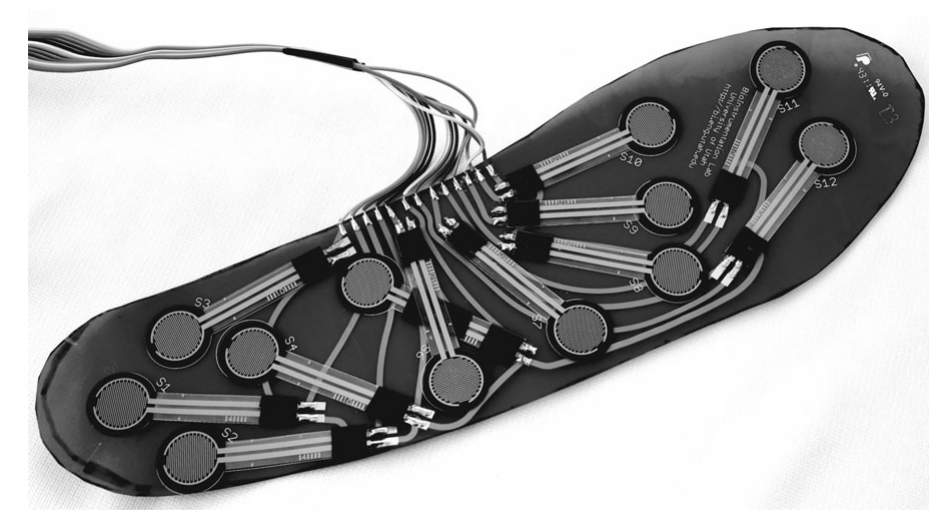

Figure 2.10: Instrumented insole containing 12 force sensitive resistors for kinetic gait analysis 9 .

Moreover, Dyer et al. [37] made use of the same force sensors to build an insole for estimation of the COP. They compared their instrumented insole with a force plate and found that it was able to track the trajectory of the COP with good correlation values, but higher errors at initial contact and during push off. However, they did not report information on the ground reaction forces and the values were obtained only from one individual.

Finally, Howell [9] developed a 12-sensor insole for kinetic gait analysis using linear regression models to determine the vertical ground reaction force and the moments corresponding to ankle dorsiflexion (or plantarflexion), knee flexion (or extension), and knee abduction (or adduction). A picture of the insole is shown on Figure 2.10. Despite the good correlation, particularly in the estimation of forces, the developed models had to be personalized for each subject, thus preventing the general use of the device without previous subject-specific calibration.

The main limitation of such systems is that they do not measure the shear components of the ground reaction force, and only a portion of the vertical components is measured, which limits the accuracy and generalization capacities of the devices. Therefore, they do not provide a concrete measurement method for "real-time" balance measurement to be used in balance enhancement projects. 


\subsubsection{Mobile force plates}

A number of research have been focused on measuring the complete ground reaction forces using a complete mobile force measurement systems or mobile force plates

Veltink et al. [38] proposed the use of two six-degree-of-freedom force and moment sensors under the shoes to measure the total ground reaction forces and center of pressures. Although their results show good correspondence compared to a force plate in terms of RMS error of the GRF and COP, the results are limited to the assumption that the sensors were flat on the ground when they were loaded. Also, when comparing the data, they did not keep track of the movement of the foot over the force plate, but optimally aligned both COP trajectories by minimizing their RMS distances. Finally, because of the added weight and the height of the system to the shoes (sensor height alone was $15.7 \mathrm{~mm}$ ) normal gait patterns could be disturbed. These sensors are also very rigid distracting the gait.

In [39], they continued the previous line of work, and investigated the modification of the gait caused by these instrumented shoes. This time, they attached markers to the instrumented shoes to track the movement of the shoes over the force plate. They found significant difference in gait pattern between three shoe types: normal, heavy and instrumented shoes. Additionally, now that the position of the shoes was tracked, the horizontal component of the ground reaction force showed a larger difference from previous results, as well as the COP trajectory.

Finally, Liu et al. 10 developed a mobile force plate composed of two small sections that are attached under the forefoot and heel sections of the shoe. Each section has three small triaxial force sensors as well as inertial sensors to measure the complete ground reaction forces and estimate the orientation of the feet for gait analysis. Figure 2.11 (a) shows the device mounted on a shoe, (b) shows the three small triaxial force sensors and (c) shows a side view of one of the sections. They 


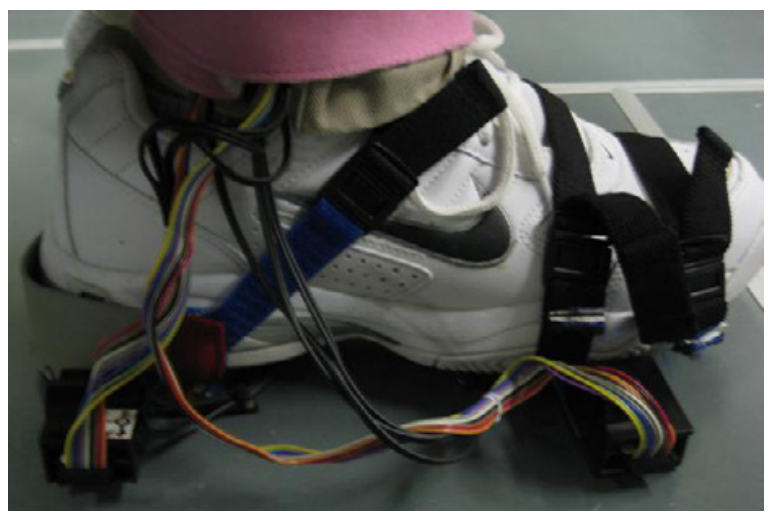

(a)

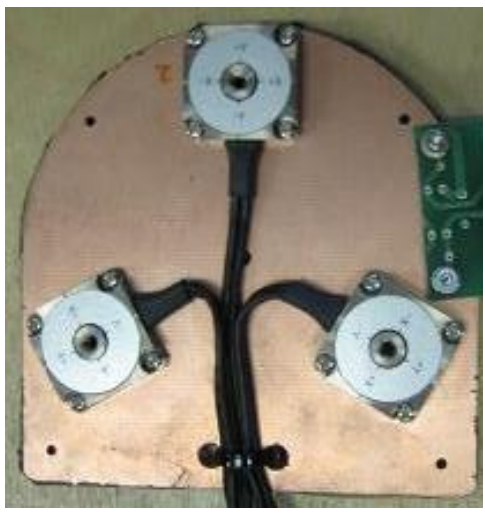

(b)

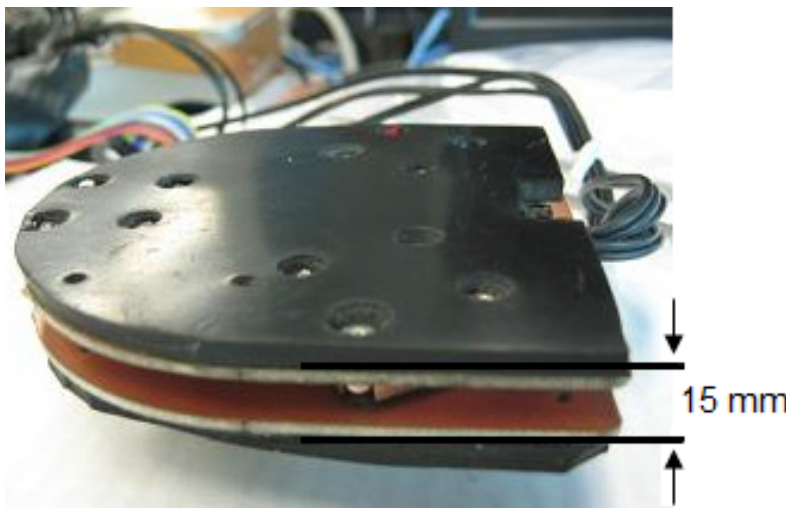

(c)

Figure 2.11: Prototype of a mobile force plate for measurement of the three components of the ground reaction force. (a) System mounted on a shoe. (b) Triaxial sensors used on each section. (c) Side view of a section showing the height of the device $[10$.

also developed a mechanism to align both sections while the device is placed on the user. The system is able to measure the three components of the ground reaction force, however, because of its $15 \mathrm{~mm}$ thickness and the lack of flexibility around the forefoot, the normal gait pattern of the user may be slightly altered. 


\subsection{Tactile feedback}

A recent research trend includes developing prosthetic devices to enhance balance for people with vestibular disorders and the elderly, such as the work done by a group in the Harvard MIT Division of Health Science and Technology [23, 47]. They developed a balance prosthesis using inertial sensors (accelerometers and gyroscopes) to measure body tilt, and vibrotactile elements mounted on a belt to display the information that is usually collected by the vestibular system to the user on the waist. The preliminary results suggested that the lateral postural sway could be reduced using the feedback information from the prosthesis during standing [25] and walking [24]. They also investigated the possible use of the device in everyday life activities by asking the participants to perform a secondary task while wearing the device [26].

This research shows the ability of the user to learn and interpret tactile feedback signals for postural control. The main limitation of this research is the size of the device, considering that its aim is to be used all day. In the study by Wall III [24], it was mentioned that sometimes the elderly subjects could not repeat some trials because they became tired. Also, since the information displayed is based only on body tilt, it may be possible that in some situations the person is performing one activity that the system declares as out of threshold, but he or she is actually in control.

\subsection{Summary}

New technology for ambulatory measurement of ground reaction forces is emerging. However, there are still two main limitations with these devices. They either only provide a partial measurement of the vertical ground reaction force, or are too rigid 
and bulky to allow normal walking and long-term use. These limitations prevent the use of these devices in a real-time balance enhancement system.

In this thesis we propose an insole that provides a measurement of the total ground reaction force and center of pressure, but is still flexible to be worn inside the shoe for long periods of time without causing discomfort or disturbing gait. This system is designed for integration with a foot localization system [40] for the BalanceAid project. 


\section{Chapter 3}

\section{System Design}

Previous attempts of inexpensive insoles using Force sensitive resistors (FSR) have measured the forces under the feet, but only a partial measurement of the total vertical force was obtained [8,36, 48], or they were only used to identify loading and unloading conditions [35]. Even in the insole by Howell [49] where 32 sensors were used to cover most of the foot area, only about $50 \%$ of the total force was recorded. The goal of the insole design thus, is to have a better measurement of the total vertical ground reaction force and center of pressure on each foot.

This chapter presents the design requirements of the insole, the selection of the sensors and the design process to investigate the number of sensors needed, their position and other design parameters of the insole. Finally, the implementation process is described along with the integration of all the components for the data acquisition.

\subsection{Design requirements}

When designing the insole for measurement of the ground reaction forces, the following objectives were identified:

- Allow normal walking without producing a change in gait patterns. 
- Be comfortable. The person should be able to wear the shoes with the insole for long periods of time without having discomfort.

- Transmit the complete forces through the sensing areas in the insole.

To address these challenges the following requirements were considered for the design of the insole:

1. Allow the normal flexion of the foot during walking, particularly during push off. The normal range of motion of the metatarsal joint is about $42^{\circ}$ as shown in Figure 3.1 .

2. Be lightweight: The maximum weight of the system should not exceed $300 \mathrm{~g}$ as recommended in [50], otherwise normal walking patterns could be compromised if the weight is in the order of $1-2 \%$ of the body weight [51].

3. Fit inside standard users shoes. In order to fit inside a shoe, the insole must be thin. Commercial insoles range between $4 \mathrm{~mm}$ to $10 \mathrm{~mm}$ thick depending on the purpose and function of the insole.

4. Have small size sensors. A maximum height of $4 \mathrm{~mm}$ and diameter of $15 \mathrm{~mm}$ is desirable so that the sensors can fit inside a shoe, and thus have a mobile and comfortable system that will not disturb normal gait.

5. Have low power consumption. This is needed to be able to have a mobile system that runs on batteries for long periods of time.

6. Withstand conditions inside the shoe, such as temperatures of $20^{\circ}$ up to $45^{\circ} \mathrm{C}$ and humidity.

7. Have minimum wiring. 

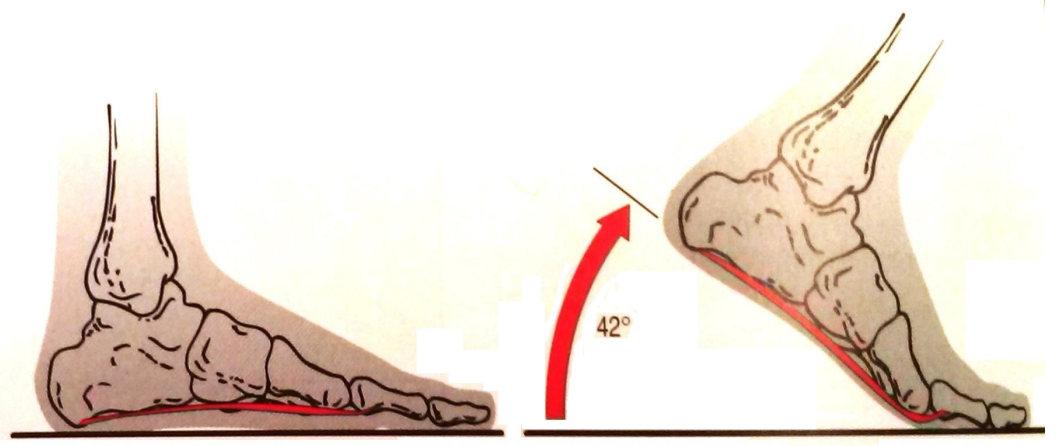

Figure 3.1: Range of motion of the metatarsal joint of the foot during push off.

8. Be affordable.

The flexibility, light-weight, and slim features will prevent any effect on gait parameters as well as helping with the comfort of the insole. One of the most important features of the insole is the transmission of the force through the sensors, because this way we will be able to get a better measurement of the GRF. The first step then was to select the proper sensors for the application.

\subsection{Sensor selection}

The general concept of the insole is as follows: force sensing elements are placed in strategic areas under the foot for measurement of the ground reaction force and small pucks are added on top of the sensors so that the force is concentrated only on the sensing area and a better measurement of the total force is obtained. Figure 3.2 shows a schematic of the position of the sensors on the insole. These positions are chosen based on the knowledge of distribution of forces under the foot from Section 3.3.2 and the results of a simulation from Section 3.3.5.

For measurement of forces, strain gauge sensors are a common solution. However, they are usually not small enough for in shoe applications, or if they come in a small size, the force range is low (within $10 \mathrm{~N}$ max). Force sensitive resistors have been 


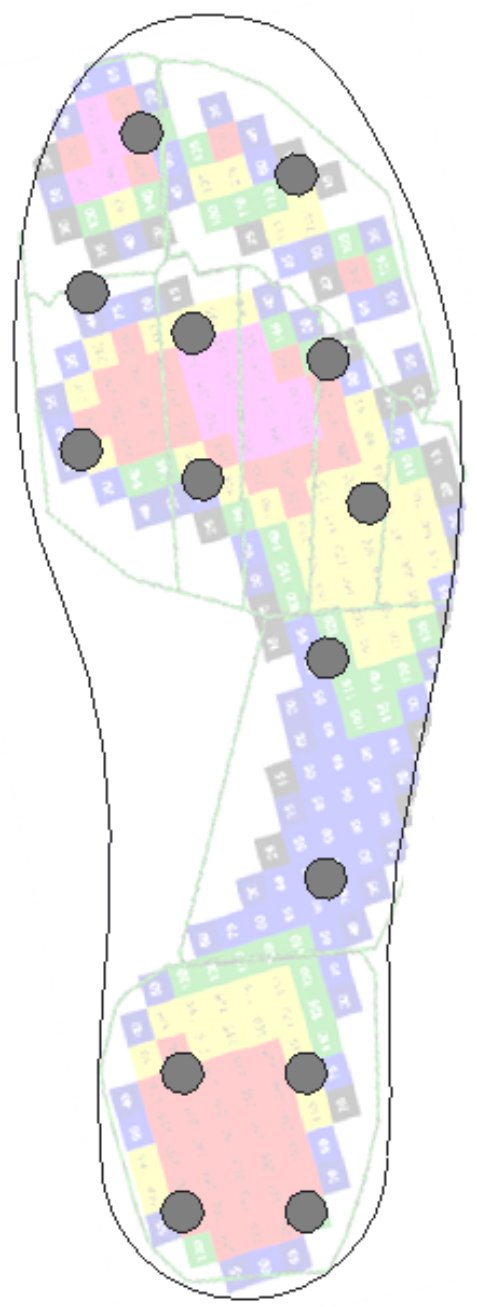

Figure 3.2: Position of sensors on insole compared with plantar pressure distribution. 
previously used for similar applications in $8,36,37,48$ for their higher flexibility and smaller size and thickness compared with strain gauge sensors. They are good for measuring load normal to their surface which goes well with the current application of measuring human ground reaction force, since it is mostly normal load. Their low cost, as well, make them suitable for the current application. However, they are limited by their accuracy and linearity, since they can present a hysteresis up to $10 \%$ and their response is nonlinear, which presents a challenge when mapping the changes on resistance to the force applied.

There are two commercially available sensors that fall in this category: The Interlink Electronics force sensitive resistors (FSR), and the FlexiForce sensors from Tekscan. Both types of force sensors work by a change of their resistance when a force is applied. Table 3.1 compares some of the characteristics of these two sensors.

Table 3.1: Comparison of characteristic of force sensitive resistors.

\begin{tabular}{|l|c|c|}
\hline & FSR & Flexiforce \\
\hline \hline Force sensitivity range & $0.2 \mathrm{~N}-20 \mathrm{~N}$ & $0-4.4 \mathrm{~N}, 100 \mathrm{~N}, 440 \mathrm{~N}$ \\
\hline Repeatability & $+/-2 \%$ & $+/-2.5 \%$ \\
\hline Hysteresis & $10 \%$ & $4.5 \%$ \\
\hline Rise time & $<3$ microseconds & $<5$ microseconds \\
\hline Temperature range & From $-40^{\circ} \mathrm{Cto} 85^{\circ} \mathrm{C}$ & From $-9^{\circ} \mathrm{Cto} 60^{\circ} \mathrm{C}$ \\
\hline Thickness & $0.3 \mathrm{~mm}$ & $0.208 \mathrm{~mm}$ \\
\hline Diameter of sensing area & $5 \mathrm{~mm}, 12.7 \mathrm{~mm}$ & $9.53 \mathrm{~mm}$ \\
\hline Linearity & nonlinear & $+/-3 \%$ \\
\hline Cost & $\$ 53$ a pack of $10(\$ 5.3$ each $)$ & $\$ 117$ pack of $8(\$ 14.6$ each $)$ \\
\hline
\end{tabular}

From Table 3.1 it can be appreciated that the FSR are less expensive than the Flexiforce sensors. However some of their characteristics make them less suitable for the current application: 
- The FSR has a nonlinear response when a load is applied, whereas the Flexiforce sensors have a linearity of $3 \%$. This property makes it easier to map the output of the sensors to the corresponding forces.

- Hysteresis for the FSR is greater, which reduces the accuracy of the sensor in dynamic situations.

- The force sensing range of the FSR sensors is only up to $20 \mathrm{~N}$, which may not be enough for the application. When a person is walking there are times when most of the weight is supported in a small area, like the toe or heel during take-off and heel-strike. As we aim to minimize the number of sensors used on the insole, we can picture a situation where a few sensors must bear most of the weight. Considering that only 5 sensors may be loaded in such cases, and an average weight of $75 \mathrm{~kg}$, it means that each sensor has to be able to measure up to $15 \mathrm{~kg}$ (about $150 \mathrm{~N}$ ). This is more than the standard range of the FSR. On the other hand, the Flexiforce sensors can measure up to $440 \mathrm{~N}$, which is enough for the application.

- Another way to compare them is with the peak pressures that the sensors can stand before damage. For the FSR this values is between 100 to 200 psi (0.69 to $1.38 \mathrm{MPa}$ ). Considering an application area of about $0.125 \mathrm{in}^{2}$ (for the 12.7 mm diameter sensor), the maximum force than can be measured by the sensor before damage is $25 \mathrm{lb}(111 \mathrm{~N})$, which is still less than the $150 \mathrm{~N}$ estimation, and which can cause damage to the sensor.

- Finally, other researchers that have previously used the FSR sensors for similar applications [49] have found that some sensors become saturated and they had to avoid placing them in areas of peak pressure.

For these reasons, the Flexiforce sensors were chosen for the current application. 


\subsubsection{Sensor description and signal conditioning}

The FlexiForce sensor shown in Figure 3.3 uses a resistive-based technology. The application of a force to the active area of the sensor results in a change in the resistance in inverse proportion to the force applied.

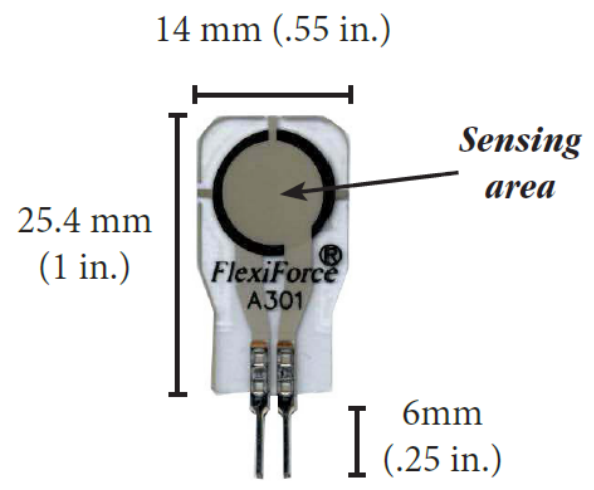

Figure 3.3: Flexiforce sensor [11].

The sensors are constructed of two layers of substrate composed of polyester film. On each layer, a conductive material is applied, followed by a layer of pressuresensitive ink. Adhesive is then used to laminate the two layers of substrate together to form the sensor. The silver circle on top of the pressure-sensitive ink defines the active sensing area. Silver extends from this sensing area to the connectors at the other end of the sensor, forming the conductive leads [11].

In order to map the changes of the resistance in the sensors to the force applied, it was necessary to implement a circuit to convert the changes in resistance to changes in voltage. A simple voltage divider could be used for this purpose. However, the change in resistance of the sensor by the force applied is nonlinear, whereas the change in conductance (the inverse property) has a linear relationship to the force applied according to the supplier's manual. For this reason, an opamp in its inverter amplifier configuration was implemented to map the changes in conductance to changes in 
voltage and then be able to relate this variable to the force applied. An MCP6004 in its inverted configuration was used in the conditioning circuit as shown in Figure 3.4 .

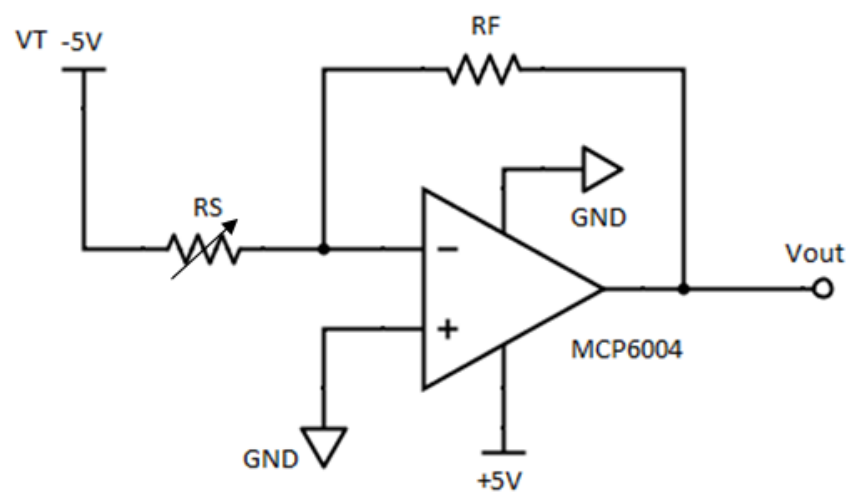

Figure 3.4: Recommended circuit for Flexiforce sensor.

The MCP6004 is a low power opamp that requires only a single supply voltage and is generally used for battery power applications due to its low current consumption of $100 \mu \mathrm{A}$. The output voltage is then a function of the change in resistance of the sensor such that

$$
V_{\text {out }}=-V_{T} * \frac{R_{F}}{R_{S}}
$$

where $V_{T}$ is the driving voltage of the sensor, $R_{S}$ is the resistance of the sensor and is a function of the applied force and $R_{F}$ is a reference resistance. The output voltage can be adjusted depending on the range of forces for the application by varying the $R_{F}$ resistor. Thus by mapping the largest force to the highest voltage $(5 \mathrm{~V})$ allowed by the $\mathrm{A} / \mathrm{D}$ we attain the maximum resolution. In this case, we set the maximum force as $200 \mathrm{~N}$. The sensor was then loaded with this force and the value of $R_{F}$ was adjusted until the output was around $5 \mathrm{~V}$. This way we prevent saturation and make use of the full voltage range. The resultant resistance value for $R_{F}$ was $18 k \Omega$.

To obtain the negative driving voltage for the sensors, an inverting switching regulator MAX764 was used with the circuit. This is a high efficiency inverter used 
commonly for battery power application since the max supply current is only $120 \mu \mathrm{A}$. It takes an input voltage from 3 to $16 \mathrm{~V}$ and produces a preset output voltage of $-5 \mathrm{~V}$ As a result, the current flowing through the sensor at full load (when $R_{S}=R_{F}$ ) was:

$$
I_{i}=\frac{5 V}{18 k \Omega}=0.277 m A
$$

which is well below the max recommended current of $2.5 \mathrm{~mA}$.

Finally, the circuit board containing the opamps and the inverter to obtain the $-5 \mathrm{~V}$ were encased on an electronic box. A picture of the complete conditioning circuit board can be seen in Figure 3.5. The complete circuit diagram is in Appendix B.

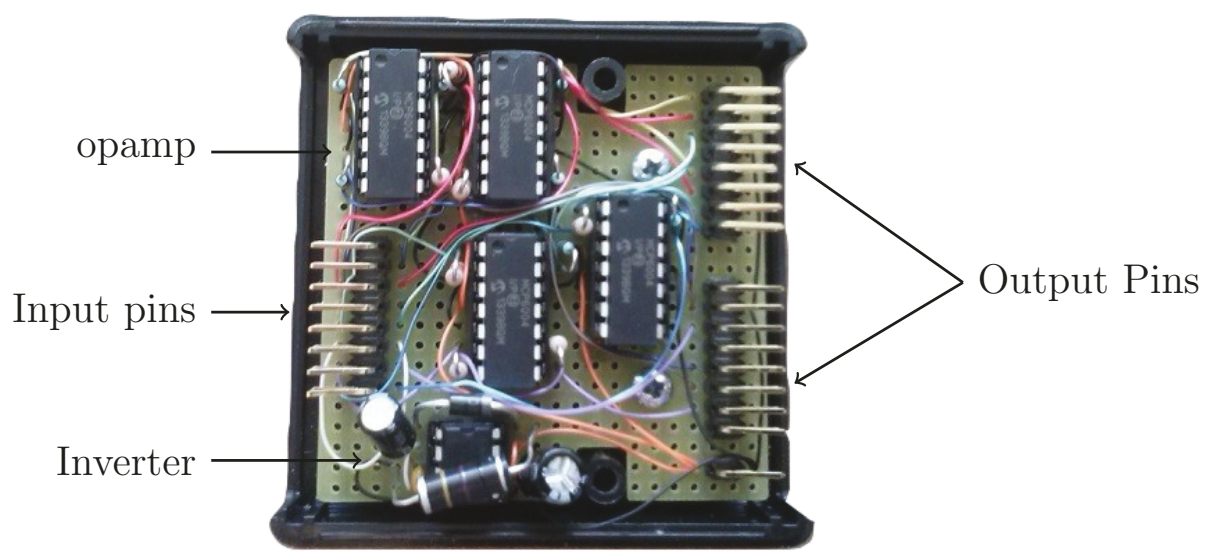

Figure 3.5: Electronic circuit board for conditioning signals from force sensors.

Once the sensors were selected, the next step was to design the insole that would contain the force sensors.

\subsection{Insole Design}

To be able to concentrate the force through the active area of the sensors, the insoles should be rigid enough to prevent excessive deflection and creating other force paths besides the puck areas. They should also be flexible enough to allow foot flexion 
Thiner sections

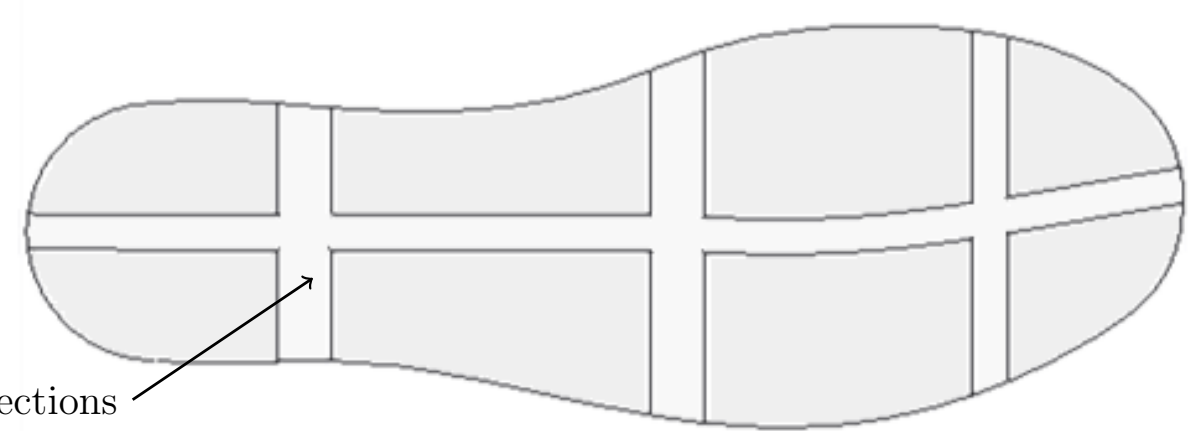

Figure 3.6: Design of the insole showing the lines of thiner sections to provide flexibility to the insole and allow the normal flexion of the foot.

particularly at the metatarsal joint, but also at the toe and midfoot. Therefore, a solution is to have flexible regions where the rigidity of the insole is modified by decreasing the thickness of the section. Such thinner areas are shown on Figure 3.6

\subsubsection{Selection of material}

Of the possible materials used for insoles, rubber was selected because it is easy to mold in any shape, and it can be harder or softer depending on the type of rubber and the thickness of the material. Some common applications for different types of rubber hardness are shown in Figure 3.7.

Rubber has a Poissons ratio of approximately 0.5. Under small deformations (less than 25\%) rubbers are linearly elastic solids, and the resulting stress to the applied strains is the same whether the strain is applied in tension or compression. Hooke's law is therefore valid within this proportionality limit. However, as the strain increases, the linear relationship is not longer valid [52], and plastic deformation could also arise, such as creep.

Since we want to minimize the thickness of the insole, polyurethane rubber with hardness A90 was selected, following the idea that a harder material will require less 
SHORE HARDNESS SCALES

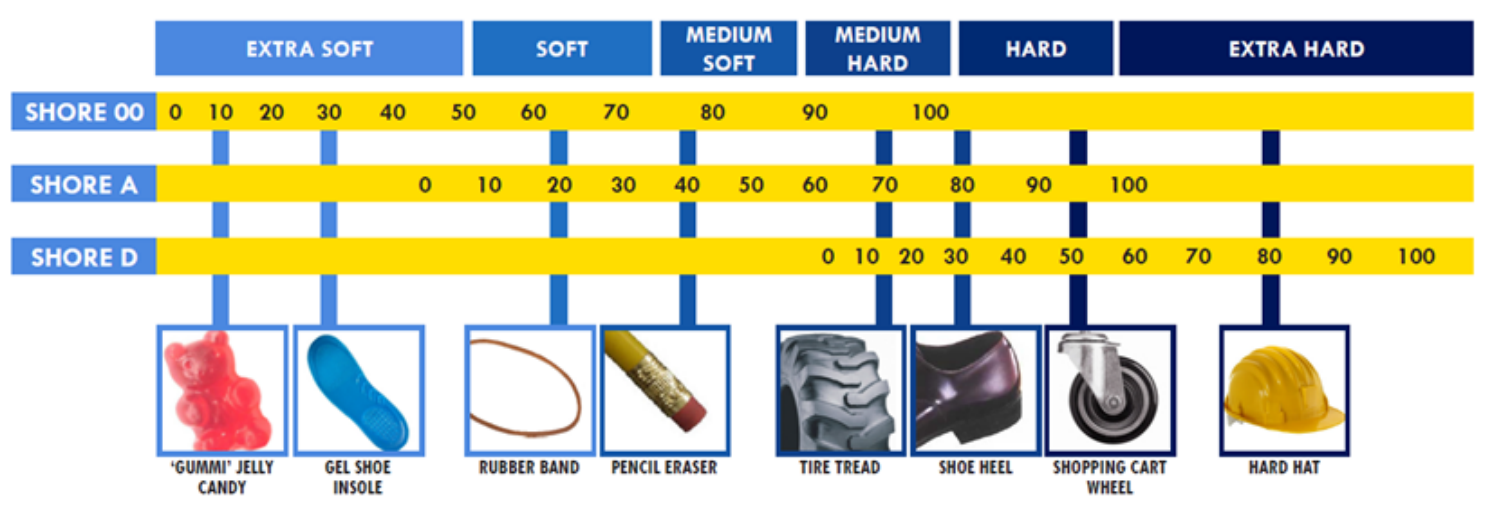

Figure 3.7: Types of rubber hardness and common applications [12].

thickness to prevent a maximum deflection since hardness depends on the stiffness of the material. This type of rubber hardness is similar to the material used for heels and outsoles of shoes. However, by adjusting the thickness in certain regions, such as between the midfoot and metatarsal as shown in Figure 3.6, we can, at the same time, make the insole flexible enough to allow normal movements such as flexion during push off.

In order to decide the final thickness of the insole and pucks, as well as the number of sensors needed and their best placement, it was necessary to investigate how the insole would deform under the load applied by the foot. For this purpose a simulation was performed in Section 3.3.5 in order to investigate the deflections cause by the forces distributed under the foot given the properties and thickness of the material.

The next sections present the information about the distribution of the forces under the foot during gait as well as the characterization of the material that was used in the simulation. 


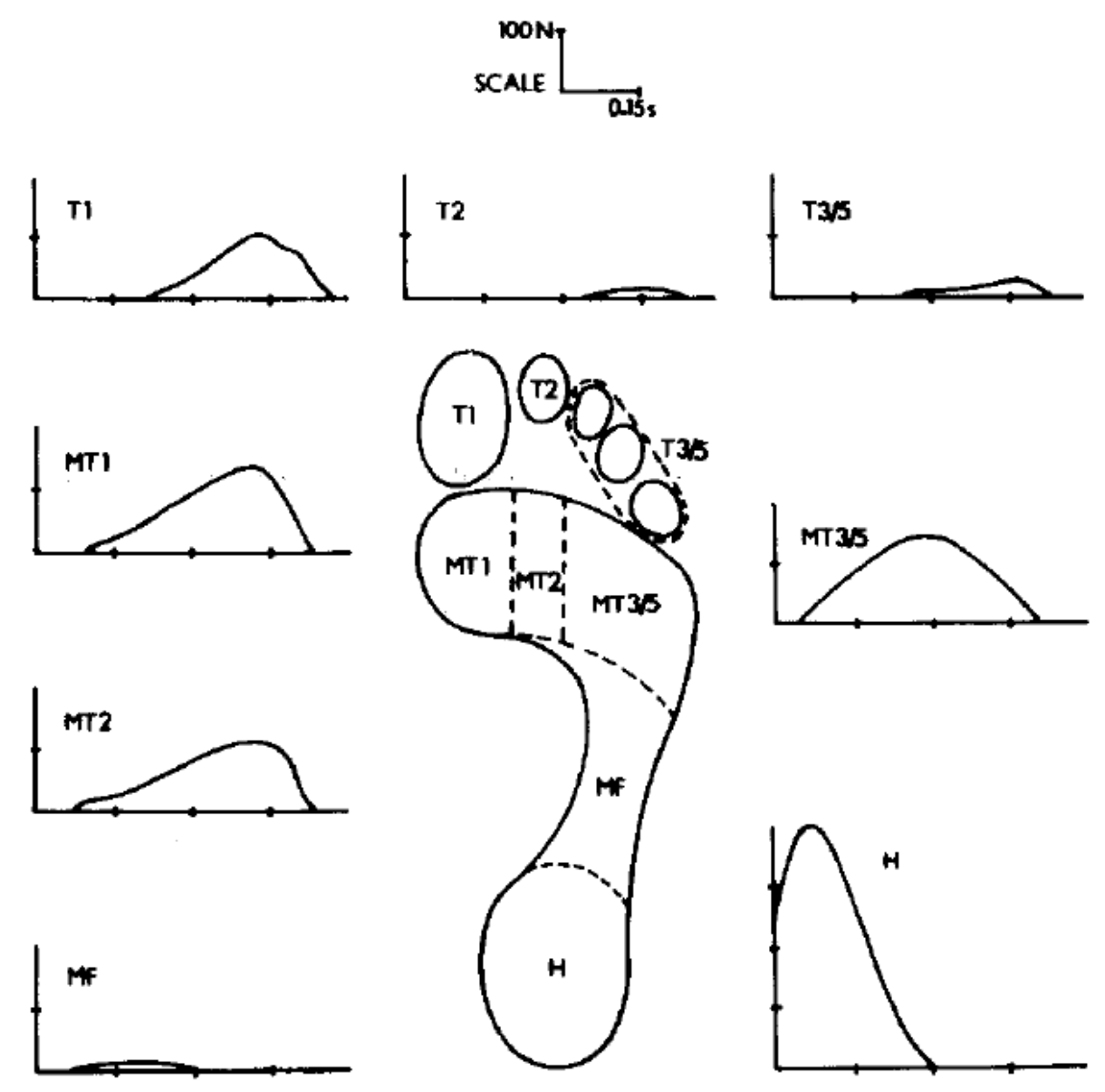

Figure 3.8: Force distribution over time under 8 different regions of the foot [13].

\subsubsection{Distribution of forces under the foot during stance phase}

Several studies have been conducted using a variety of technologies to investigate the force and pressure distribution under different regions of the foot for different applications. One of the early studies by Hutton \& Dhanendran [13] used arrays of strain gauged load cells over several surface areas to measure the distribution of forces in 8 different regions of the foot: first metatarsal, second metatarsal, third to fifth metatarsals, big toe, second toe, third to fifth toes, midfoot and heel during the stance phase of the gait cycle. Figure 3.8 shows the plots of the forces on each region 
over time. It can be appreciated that the heel area is the first to receive the body weight and as other regions of the foot get in contact with the floor, the weight is being transfered from heel to toes. However, the peak forces in each area are different.

Later, Hayafune et al. [1] reported the forces and pressure distribution at the push off phase. This is a critical phase of the gait cycle since this is the time where the second peak of the vertical ground reaction force occurs and the heel has already left the floor, thus concentrating a great force on the ball of the foot. The reported average forces between subjects, at this time of the gait cycle, on the different foot areas normalized by body weight are shown in Table 3.2 .

Table 3.2: Average force distribution between subjects during push-off phase in gait as percentage of body weight [1].

\begin{tabular}{|c|c|}
\hline \multicolumn{2}{|c|}{ Force Distribution (\% BW) } \\
\hline MT1 & 29.1 \\
\hline MT2 & 28.3 \\
\hline MT3 & 22.3 \\
\hline MT4 & 10.3 \\
\hline MT5 & 3.5 \\
\hline Big toe & 23.8 \\
\hline Toe 2 & 5.0 \\
\hline Toes 3-5 & 4.6 \\
\hline Total & 126.7 \\
\hline
\end{tabular}

Furthermore, Ledoux \& Hillstrom [14] reported the forces under different sections of the foot as function of the percentage of stance. The sections they considered were the heel, the first to fifth metatarsals and the big toe. They reported the force versus percentage of stance, as well as the force and pressure peak values in each region 


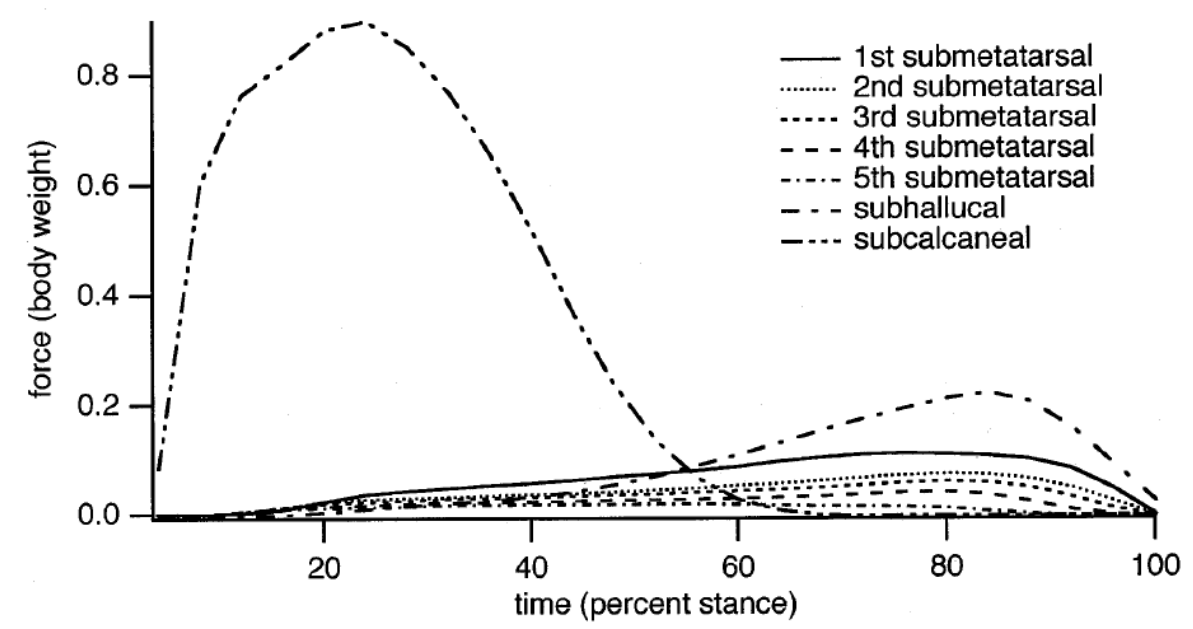

Figure 3.9: Average forces under the foot versus percent stance for 10 trials of a typical subject $[14$.

and the time of occurrence. Figure 3.9 shows the average forces for a typical subject normalized by body weight.

Lastly, Putti et al. [2] presented a study with the maximum and mean force and pressure values in men and women in 10 different regions of the foot: first, second, third, fourth and fifth metatarsals, big toe, second toe, third to fifth toes, midfoot and heel. Table 3.3 shows the maximum force values reported in their study for men and women.

Combining information from these studies, we were able to come up with an estimate of the average forces experienced on the different regions of the foot at different times of the stance phase of gait. Seven different scenarios were used in the simulation in Section 3.3.5 at 10, 20, 30, 50, 70, 80 and $90 \%$ of time of the stance phase. The foot was divided in 10 different regions as shown in Figure 3.10: first, second, third, fourth and fifth metatarsals, big toe, second toe, third to fifth toes, midfoot and heel. 
Table 3.3: Maximum force values of men and women in 10 regions of the foot 2 .

\begin{tabular}{|c|c|c|}
\hline \multicolumn{3}{|c|}{ Maximum force $(\mathbf{N})$} \\
\hline & Women & Men \\
\hline heel & 525 & 701 \\
\hline mid foot & 97 & 187 \\
\hline 1st MT & 168 & 216 \\
\hline 2nd MT & 170 & 220 \\
\hline 3rd MT & 77 & 115 \\
\hline 4th MT & 46 & 79 \\
\hline 5th MT & 41 & 72 \\
\hline Hallux & 91 & 149 \\
\hline 2nd toe & 47 & 64 \\
\hline 3-5 toes & 36 & 62 \\
\hline
\end{tabular}

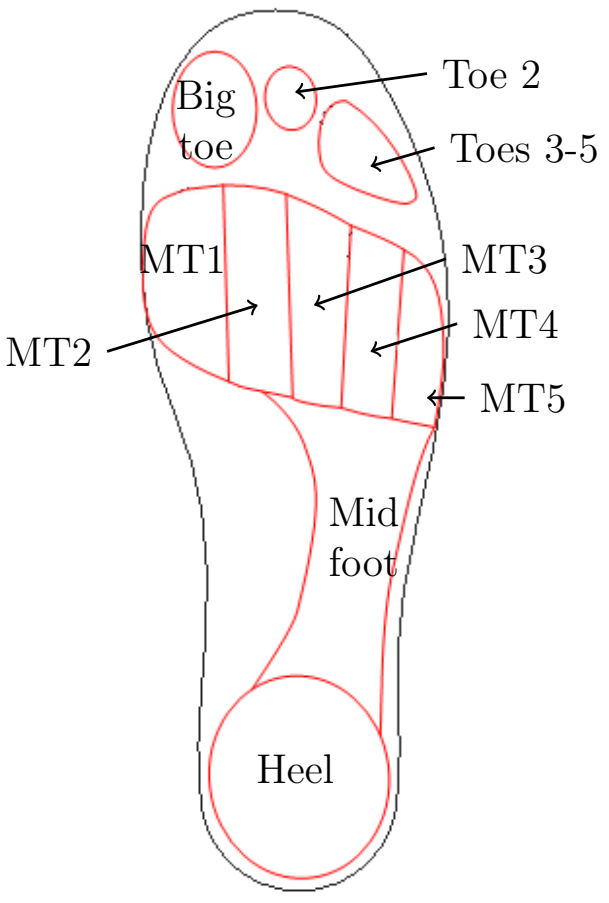

Figure 3.10: The 10 different regions of the foot used for the application of the loads for the simulation. 
For the $80 \%$ stance scenario, the values at push off phase from [1] were used, which were greater than those reported from other studies. For other phases, the values from [14 for the heel and metatarsal areas were used in combination with the values from [13] and [2], since the midfoot and toes area were not reported in [14].

All information was given as percentage of body weight and was used later to simulate the forces under the foot for the design of the insole in Section 3.3.5. The final values chosen in each scenario are shown in Table 3.4 .

Table 3.4: Plantar vertical force distribution values used for simulation as percentage of body weight.

\begin{tabular}{|l|c|c|c|c|c|c|c|}
\hline & \multicolumn{7}{|c|}{ Percentage of stance } \\
\hline \hline Foot area & $10 \%$ & $20 \%$ & $30 \%$ & $50 \%$ & $70 \%$ & $80 \%$ & $90 \%$ \\
\hline heel & 70.0 & 87.8 & 80.0 & 20.0 & 1.0 & & \\
\hline midfoot & & 10.0 & 16.2 & 8.0 & 2.0 & & \\
\hline MT1 & & 3.3 & 5.5 & 15.0 & 25.7 & 29.1 & 10.8 \\
\hline MT2 & & 2.8 & 4.0 & 13.1 & 22.2 & 28.3 & 10.2 \\
\hline MT3 & & 3.0 & 4.0 & 12.5 & 19.4 & 22.3 & 5.7 \\
\hline MT4 & & 2.8 & 3.4 & 7.9 & 10.8 & 10.3 & 2.2 \\
\hline MT5 & 0.7 & 2.2 & 2.7 & 4.7 & 5.1 & 3.5 & 0.3 \\
\hline Big toe & & & 1.0 & 6.0 & 15.6 & 23.8 & 17.5 \\
\hline toe 2 & & & & 1.5 & 4.0 & 5.0 & 2.0 \\
\hline toes 3-5 & & & & 1.0 & 3.0 & 4.6 & 1.5 \\
\hline Total & $70.7 \%$ & $111.9 \%$ & $116.8 \%$ & $89.5 \%$ & $108.8 \%$ & $126.9 \%$ & $50.2 \%$ \\
\hline
\end{tabular}

As can be noted in Table 3.4 the total force is not $100 \%$ at all times, this is because the GRF exhibits a double hump due to the acceleration and deceleration of the COM as explained in Section 2.2.1.

One limitation of the current approach is that the division of plantar areas considered by each study are not exactly the same, as a consequence, the values of the 
forces reported on each area varies as well. For the simulations, we considered the same division of areas as in [1] and when comparing different papers, the maximum values for each area were used. As such, this can only be considered a general estimation of the forces, since there will always be variability between subjects, but the values can still give an approximation of the forces and provide valuable information for simulation.

\subsubsection{Number of sensors}

When studying the contact regions of the foot, it becomes apparent that there are two main regions that carry most of the body weight during single stance: the heel and the metatarsal zone. Therefore at least 3 sensors are recommended to be used in these areas to distribute the load and also to avoid load paths outside the sensors area. In addition, there are two other regions that are loaded at different stance times, thus providing relevant information about the distribution of the forces: the big toe and the midfoot. Consequently, two more sensors were used in these areas. Finally an extra sensor can be added in the area of the remaining toes to increase the accuracy of the calculation of the COP during push off phase. This results in a total of minimum 9 sensors. The aim of the simulation in Section 3.3.5 then is to investigate the need of extra sensors and their positions, taking into account the known regions of higher pressure.

\subsubsection{Sensor material characterization}

Once the distribution of forces under the foot was investigated and materials selected, samples of the rubber were created for characterization experiments to obtain properties required by the simulation. Cylindrical samples were selected according to the D575 ASTM standard for rubber testing in compression. Three specimens were 
built for testing. Because the samples were made in a manual molding process, the resulting samples had different sizes. Their final dimensions are shown in Table 3.5 .

Table 3.5: Final dimensions of rubber samples for compressive test.

\begin{tabular}{|c|c|}
\hline Diameter $(\mathrm{mm})$ & Thickness $(\mathrm{mm})$ \\
\hline 30.01 & 15.91 \\
\hline 30.15 & 15.57 \\
\hline 29.94 & 15.59 \\
\hline
\end{tabular}

In each experiment, compressive forces were applied and removed in three successive cycles according to the standard. The first two cycles were for the purpose of conditioning the specimen, and the readings were taken during the third application of force. The force was applied to produce a deflection rate of $12 \mathrm{~mm} / \mathrm{min}$ until a deflection of $20 \%$ was reached, after which the force was released immediately at the same rate. A MTS machine was used to perform the tests. The set up is shown in Figure 3.11. Two metal plates were attached to the grips in order to apply the compressive load over the sample. Additionally, sandpaper was placed between the rubber specimen and the metal plates to avoid lateral slippage of the rubber.

The sample data taken from the three specimens from the test is shown in Figure 3.12. The average stress and strain values used as inputs to model the material for the simulation are shown on Table 3.6.

\subsubsection{Simulation and final design}

With the information from Section 3.3.2 and Section 3.3.4 about plantar force distribution, contact areas of the foot and properties of the rubber material a simulation was performed. The objectives of the simulation were to investigate: 


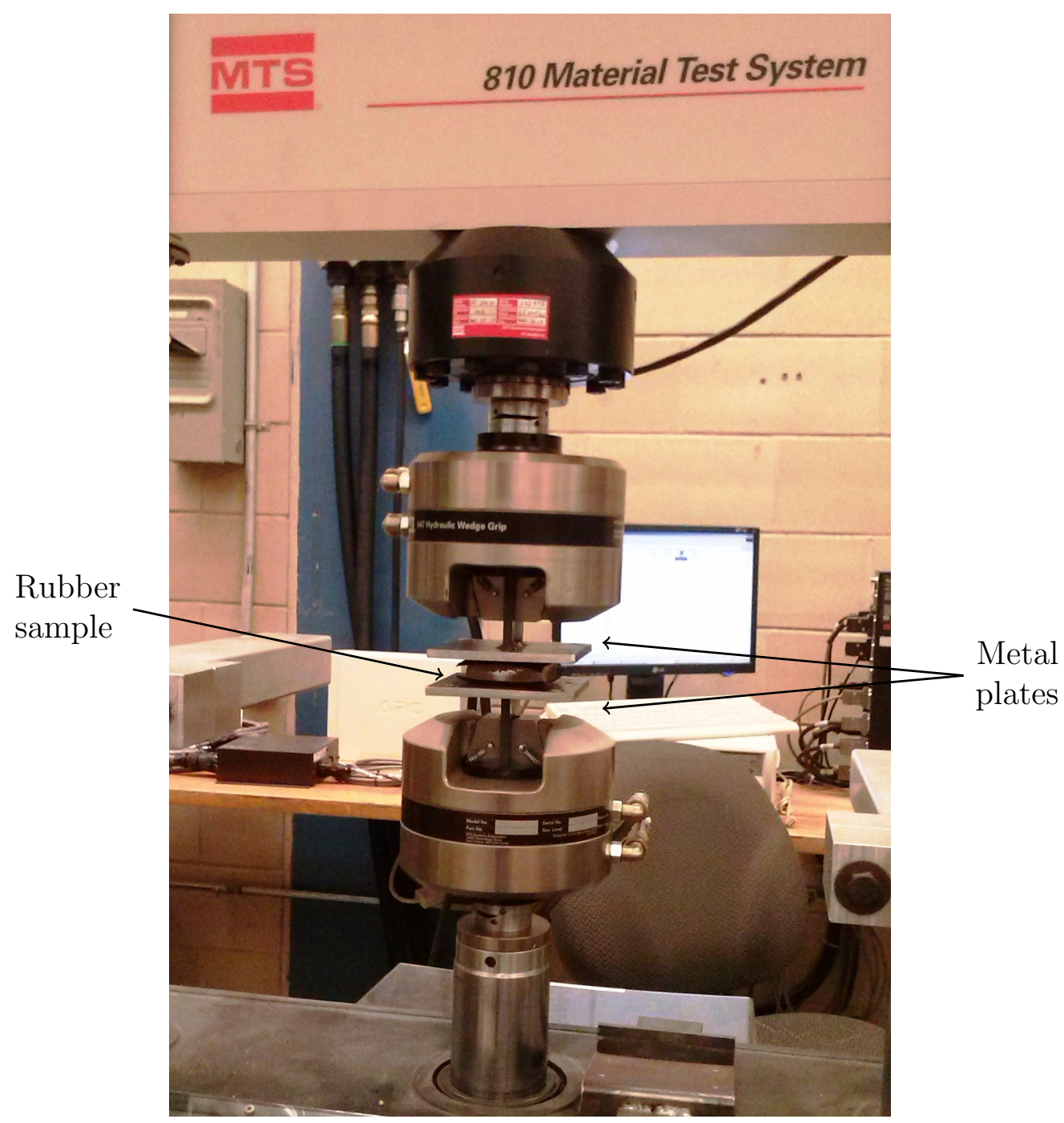

Figure 3.11: Rubber sample in MTS machine for compressive test. 


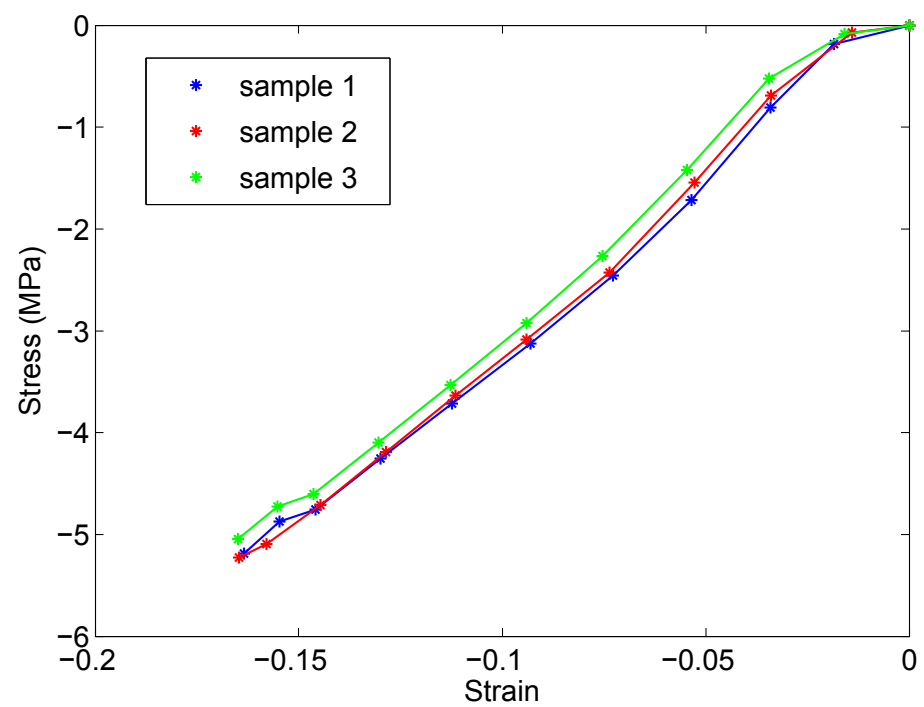

Figure 3.12: Stress-Strain curves for the three rubber samples under uniaxial compression.

Table 3.6: Average stress and strain values from the compression test used to characterize material.

\begin{tabular}{|c|c|}
\hline Strain & Stress (MPa) \\
\hline \hline-0.0141 & -0.0735 \\
\hline-0.0340 & -0.6876 \\
\hline-0.0528 & -1.5457 \\
\hline-0.0736 & -2.4301 \\
\hline-0.0940 & -3.0860 \\
\hline-0.1115 & -3.6382 \\
\hline-0.1286 & -4.1907 \\
\hline-0.1447 & -4.7103 \\
\hline-0.1579 & -5.0925 \\
\hline-0.1647 & -5.2249 \\
\hline
\end{tabular}


1. The proper number of sensors needed for the total measurement of vertical ground reaction force.

2. The position of sensors.

3. The thickness of insole and pucks.

The design goal was to minimize the total thickness of the insole while making sure the deflections remained smaller than the thickness of the pucks, thus preventing the creation of alternative force paths beside the pucks.

For the simulation, the following parameters were required as inputs for the model:

1. Distribution of forces under the foot at different stance times. This information was described in Section 3.3.2.

2. Contact areas of the foot were the load is applied. This information was also presented in Section 3.3 .2 .

3. Properties of the rubber material selected. This was investigated in Section 3.3.4

4. Constraints of the model. Displacement constraints were applied at the puck areas of the model.

The outputs of interest from the simulation were the magnitude and regions of the deformation. This information was used to iterate over the design and modify the number and location of the sensors, as well as the thickness of the insole and pucks.

To design the insole, the CREO sofware for CAD design was used along with the SIMULATE module for static simulation of the forces.

The stress-strain data obtained from the compressive test on rubber was used as input parameters to characterize the hyperelastic behaviour of the material. The data was input as a uniaxial test in CREO SIMULATE and the software compared and selected the hyperlastic model that best fit the data. Some of the hyperelastic models 


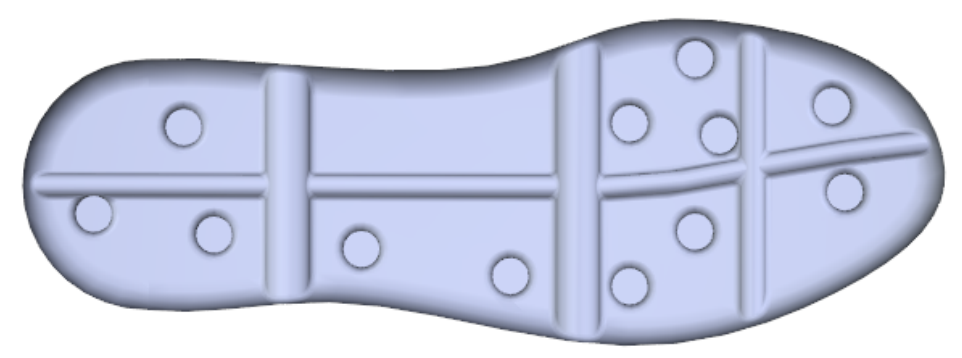

Figure 3.13: Design of first model of the insole showing the position of pucks and thinner sections.

available were: Mooney-Rivlin, Neo-Hookean, Polynomial Order 2 and Yeoh model. In this particular case, the Polynomial Order 2 was the best fit for the data provided.

One of the limitations of the current approach is that data from only a uniaxial test was used to characterize the material, whereas for rubber and other hyperelastic materials, bidirectional tests are also recommended for a more accurate model. However, this limitation is mitigated by the fact that the level of the strains on the material remained low (below 25\%) and in this region the material behaves similarly in tension and compression and has a linear relationship between strain and stress, as can be appreciated in the plot of the data in Figure 3.12

For each stance phase a static simulation was run with the properties of the material loaded as explained above and the values of forces from Table 3.4 . For the simulation, we assumed an average weight of $70 \mathrm{~kg}$.

The first model was developed using 12 sensors: 3 on the heel, 2 on the midfoot, 5 on the metatarsal zone, 1 on the big toe, and 1 over the other toes. The position of the sensors over the surface of the insole were located close to the regions of peak pressure to decrease the deflection on those areas and allow the force to be measured through the force sensors. Figure 3.13 shows the design of the first model. 
The thickness of the insole was used as the variable to modify and compare the resulting deformations in the insole. This parameter was iterated in order to find a minimum thickness that, summed up with the height of the pucks, will avoid the creation of other load paths due to the deformations.

The outcome of the first simulation was a model with a total thickness of $8 \mathrm{~mm}$ (insole thickness of $5 \mathrm{~mm}$ plus $3 \mathrm{~mm}$ pucks) to prevent displacements greater than $2 \mathrm{~mm}$ when a normal force was applied. Additionally, by adding thinner sections between the midfoot and the metatarsal as described in Section 3.3.1, it also allowed the flexion during push off.

Then, the first prototype of the insole was made and preliminary experiments were carried out as presented in Chapter 4. These experiments revealed the need for an extra sensor on the heel, since the forces recorded when the weight was shifted to the back of the insole were lower than those registered by the force plate.

A final model of the insole was then made considering the experience with this first prototype, and the simulation was updated. The maximum displacements at each percentage of stance phase for the final version are shown in Table 3.7

Table 3.7: Resulting insole displacements from simulation of plantar forces at different percentage of stance.

\begin{tabular}{|c|c|}
\hline Stance & Max Displacement $(\mathbf{m m})$ \\
\hline \hline $10 \%$ & 1.77 \\
\hline $20 \%$ & 2.14 \\
\hline $30 \%$ & 1.98 \\
\hline $50 \%$ & 0.94 \\
\hline $70 \%$ & 1.40 \\
\hline $80 \%$ & 1.72 \\
\hline $90 \%$ & 1.22 \\
\hline
\end{tabular}




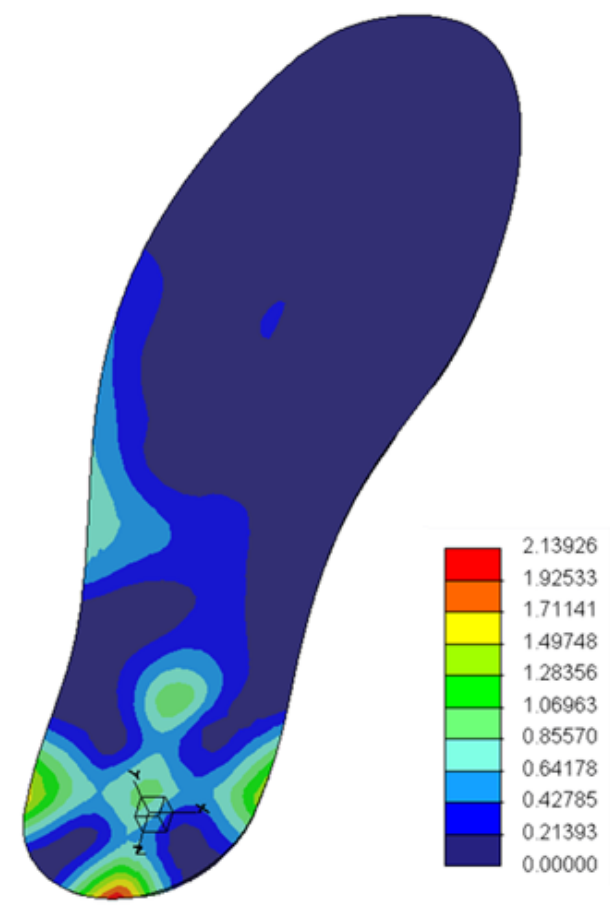

(a) Heel strike (20\% of stance)

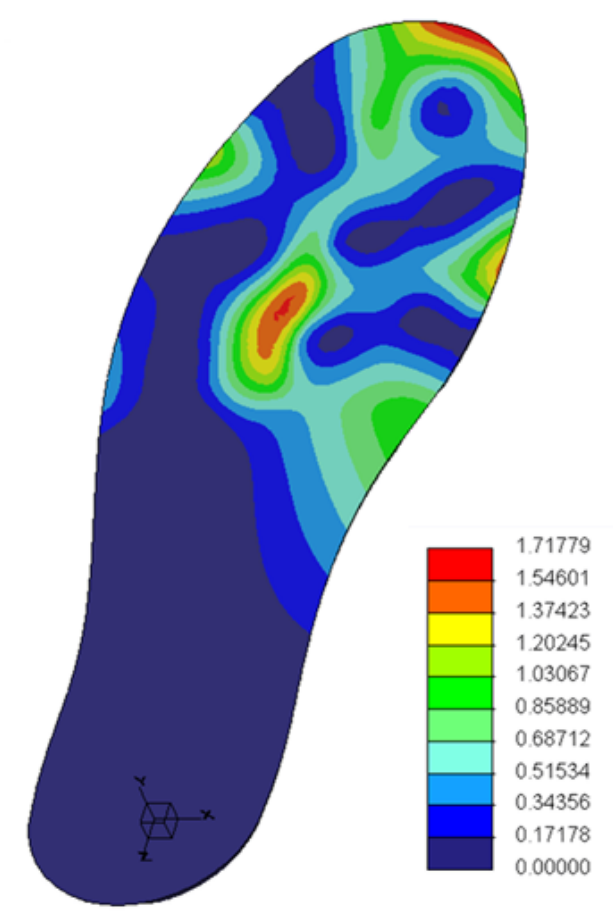

(b) Push off ( $80 \%$ of stance)

Figure 3.14: Displacement simulation results for heel strike and push off times.

The displacement graphs for the final simulations for the two most critical scenarios (heel-strike and push-off) are shown in Figure 3.14. The greater displacements were observed in the back of the heel at initial contact during heel strike and at the metatarsal zone during push off.

From Table 3.7 the max displacement for the final design was $2.1 \mathrm{~mm}$ for an insole thickness of $4.5 \mathrm{~mm}$. Hence, the resulting thickness of the insole was $7 \mathrm{~mm}(4.5 \mathrm{~mm}$ thickness plus $2.5 \mathrm{~mm}$ for puck areas) with 14 sensors distributed as follow: 4 in the heel, 6 on the metatarsal area, 2 on the midfoot, 1 on the big toe, and 1 on the other toes. By adding the two extra sensors we reduced the displacements of the insole, specially on the heel area, but we were also able to decrease the required thickness of the insole, since the number of contact points to support the load increased. 


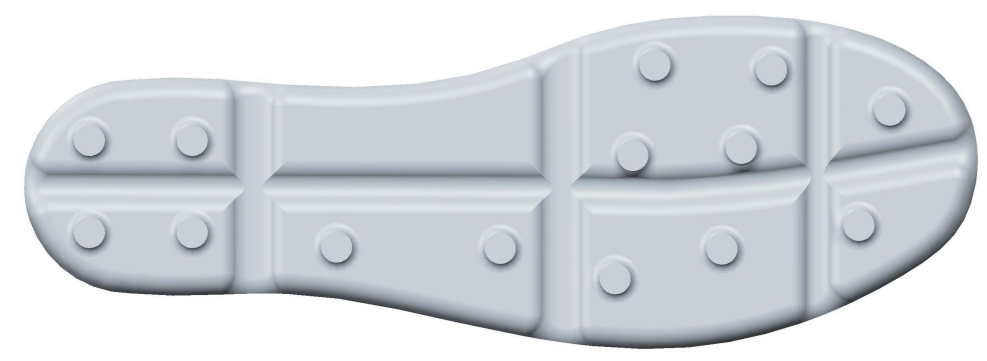

(a) Bottom view

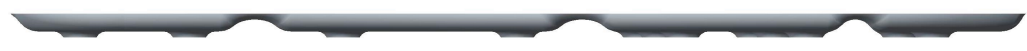

(b) Right view

Figure 3.15: Final insole design showing position of pucks and thinner sections.

Finally, thinner sections at the center line of the insole, and between the heel and midfoot zone, midfoot and metatarsal zone and metatarsal and toes zones were added to give flexibility to the insole and allow normal flexion during walking. The final design of the insole is shown in Figure 3.15

The origin of the coordinate system in the insole was located at the intersection between the center line and the thinner section between the midfoot and the metatarsal zone as shown in Figure 3.16. The final positions of the sensors with respect to the coordinate system in the insole are shown in Table 3.8

\subsubsection{Implementation}

To make the insoles, molds were machined from aluminum for the casting of the liquid rubber as shown in Figure 3.17. The final insole was made of polyurethane rubber hardness A90 from Smooth-On following the instructions from the datasheet from the supplier. This rubber has a working time of $20 \mathrm{~min}$ once the two liquid components are mixed. For the casting, a release agent was applied to the mold to 


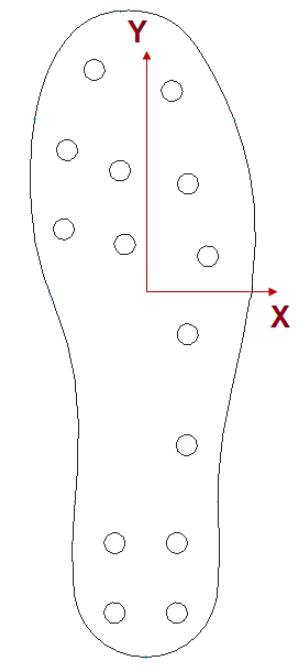

Figure 3.16: Coordinate system on the insole.

Table 3.8: Position of sensors in the insole frame.

\begin{tabular}{|r|r|}
\hline $\mathbf{X}(\mathbf{m m})$ & $\mathbf{Y}(\mathbf{m m})$ \\
\hline 12.50 & -128.49 \\
\hline-12.50 & -128.49 \\
\hline 12.50 & -100.49 \\
\hline-12.50 & -100.49 \\
\hline 16.44 & -61.13 \\
\hline 16.80 & -16.62 \\
\hline 25.03 & 14.67 \\
\hline-8.41 & 19.48 \\
\hline-32.95 & 25.50 \\
\hline 16.98 & 43.74 \\
\hline-10.36 & 49.02 \\
\hline-31.65 & 57.24 \\
\hline 10.56 & 81.05 \\
\hline-20.74 & 89.47 \\
\hline
\end{tabular}




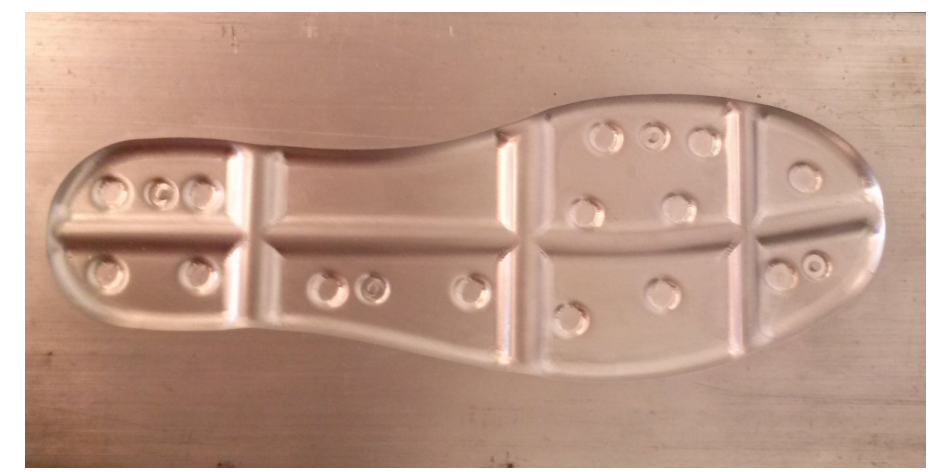

Figure 3.17: Mold used for casting the rubber for the top part of the insole

facilitate demolding and a vacuum chamber was used for vacuum degassing to reduce entrapped air bubbles.

Two insole parts were made, a top one, with the calculated thickness of $4.5 \mathrm{~mm}$, and a thin $1 \mathrm{~mm}$ bottom part to cover and protect the sensors. Because the sensors work better when they are placed over a smooth flat surface, and in order to prevent their bending due to indentation once inside the shoes, the $2.5 \mathrm{~mm}$ puck section was divided between the top and bottom parts of the insole, resulting in a top part with pucks of $1.5 \mathrm{~mm}$ thick, and a lower part with pucks of $1 \mathrm{~mm}$. The 14 force sensors were then placed and glued between the puck areas of both layers as shown in Figure 3.18 . Four pins and holes were added to the bottom and top sections to align both insole parts and tape was used to hold the layers together.

One of the challenges of building the insole was to minimize the wiring. To accomplish this, thin wires were connected to the sensors from the supply voltage and a single ribbon cable carrying the signals from the sensors and coming out from the lateral region of the insole was connected to the conditioning circuit board. The final prototype can be seen on Figure 3.19 


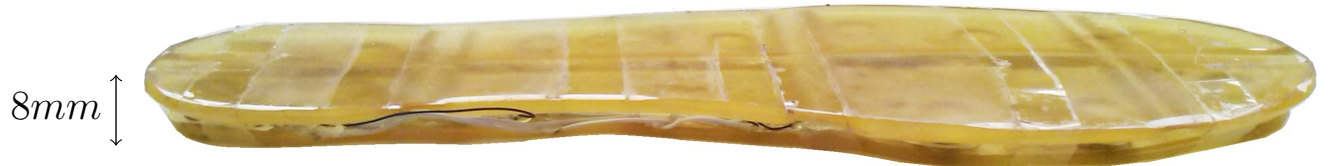

(a)

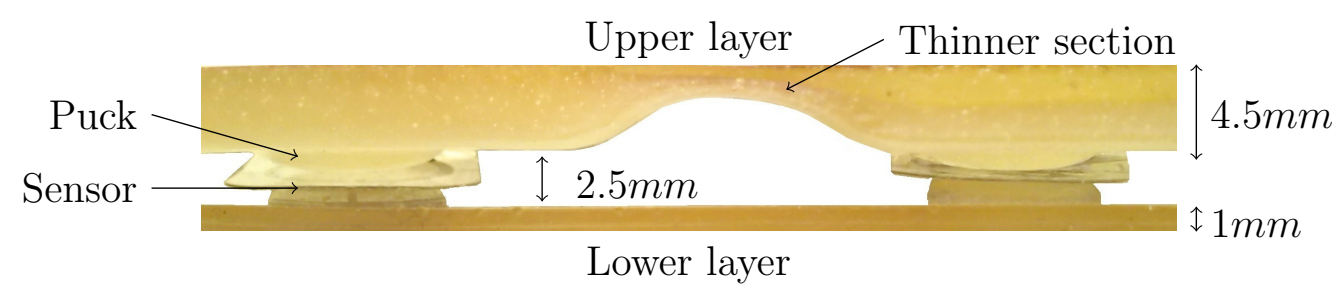

(b)

Figure 3.18: (a) Side view of the final insole showing the total thickness. (b) Enlarged view of the cross section of the insole showing the placement of the sensors between the pucks of the two layers of the insole.

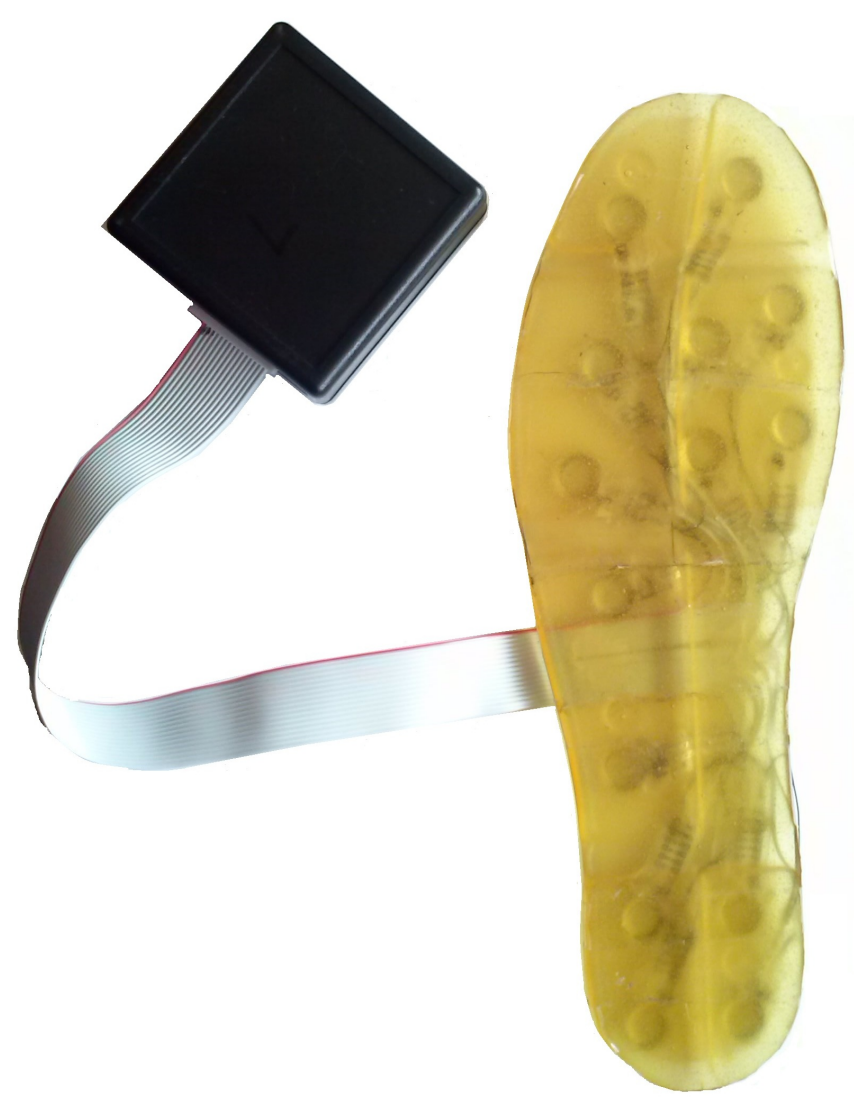

Figure 3.19: Final rubber insole with embedded force sensors. 


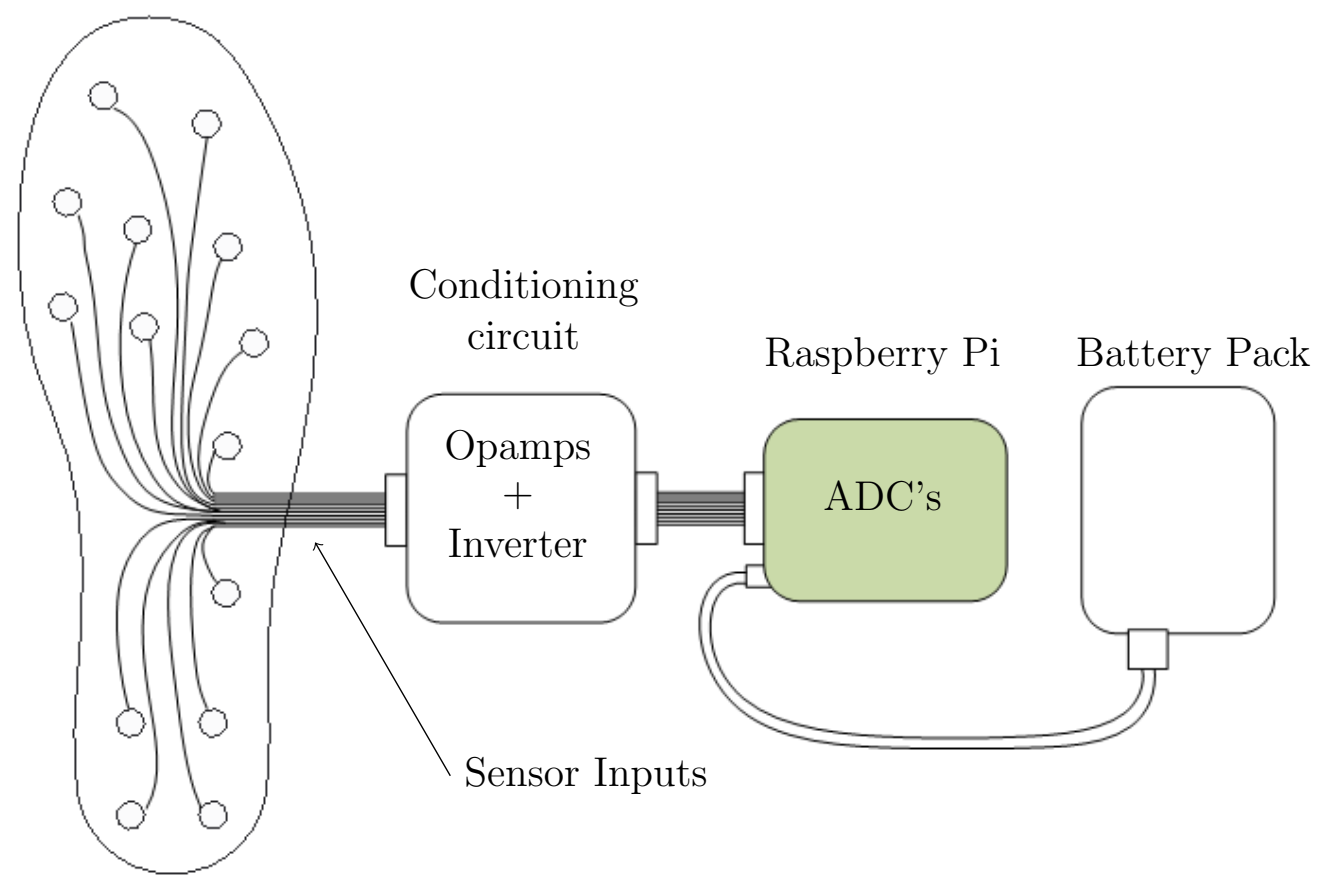

Figure 3.20: Components of data acquisition system.

The conditioning circuit containing the opamps and the inverter to obtain the $-5 \mathrm{~V}$ with low current consumption was encased in an electronic case that was attached to the ankle of the user by means of a velcro strap. The analog output voltage was then connected to the data acquisition system. A schematic of the components is shown in Figure 3.20

\subsection{Data acquisition system}

For collection, processing and integration of data for the project, a Raspberry Pi was chosen as the platform to work with. The Raspberry Pi is a single board computer the size of a credit card and costs only US $\$ 35$. Its small size and low cost make it suitable for the mobile application in this project. As a result, there is no need for any external computer for processing the data and integration of signals with the 


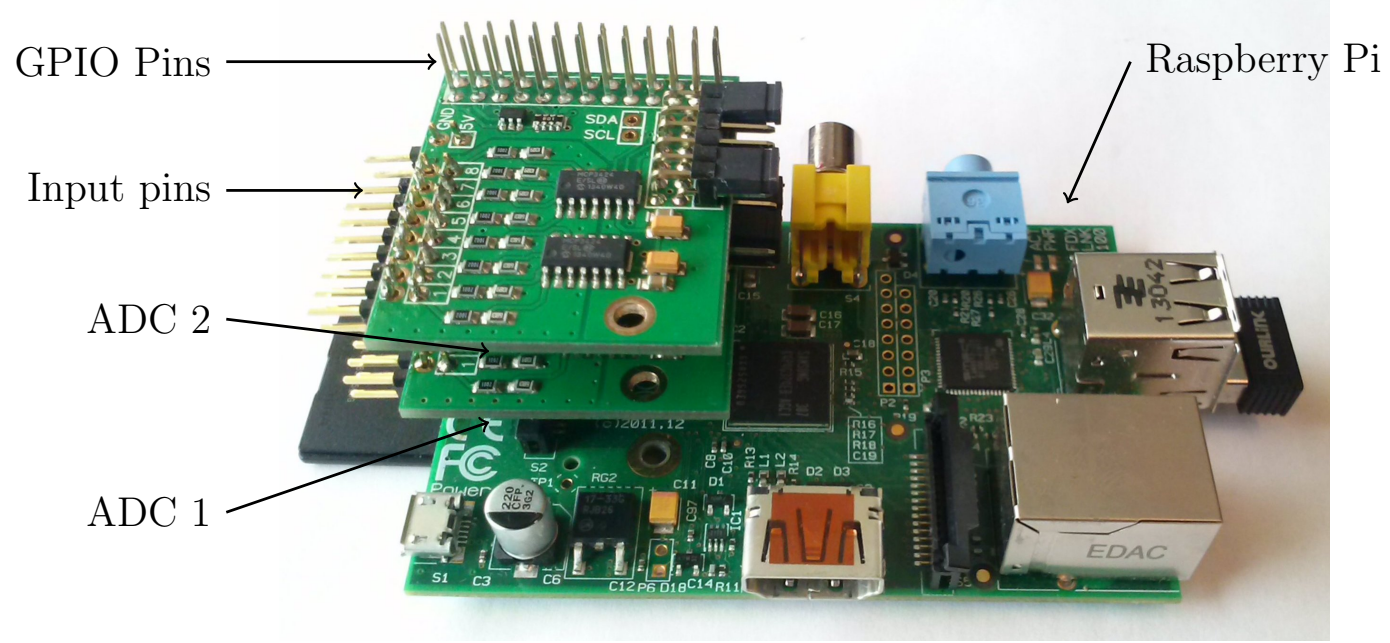

Figure 3.21: Analog to digital converters mounted on Raspberry Pi.

foot localization system from [40]. However, the Raspberry Pi does not come with integrated I/O analog pins to acquire signals from sensors.

Two external boards with 8 A/D channels each designed for use on the Raspberry Pi were acquired from ABelectronics as shown in Figure 3.21. These boards use 2 MCP3424 low noise analog to digital converters from Microship, each with an on board programmable gain amplifier (PGA) and programmable data rate options up to 240 samples per second (SPS).

However, this speed is only available for continuously reading one channel. Because of the multiplexer on the ADC chip, speed is compromised once the 4 channels are to be used leading to a max of 60 SPS if using the 4 channels on each chip. Two boards were used for each shoe, for a total of 16 available inputs on each side.

According to a study of the frequency content of gait [53], 98\% of the power of the vertical ground reaction force signal is below $10 \mathrm{~Hz}$, and $99 \%$ below $15 \mathrm{~Hz}$. As a result, the minimum sampling rate for measuring ground reaction forces should be greater than $30 \mathrm{~Hz}$. For this application, a sampling rate of $50 \mathrm{~Hz}$ that is sufficient was achieved. 
Finally, a rechargeable lithium-polymer battery pack was used to provide mobile power for the Raspberry Pi and the circuit board. The battery pack has a capacity of $6600 \mathrm{mAh}$ and provides $5 \mathrm{~V} / 1 \mathrm{~A}$ at one port and $5 \mathrm{~V} / 2 \mathrm{~A}$ at the other. The battery pack is $22.5 \times 62 \times 97 \mathrm{~mm}$ in size and is shown on Figure 3.22 alongside a quarter coin.
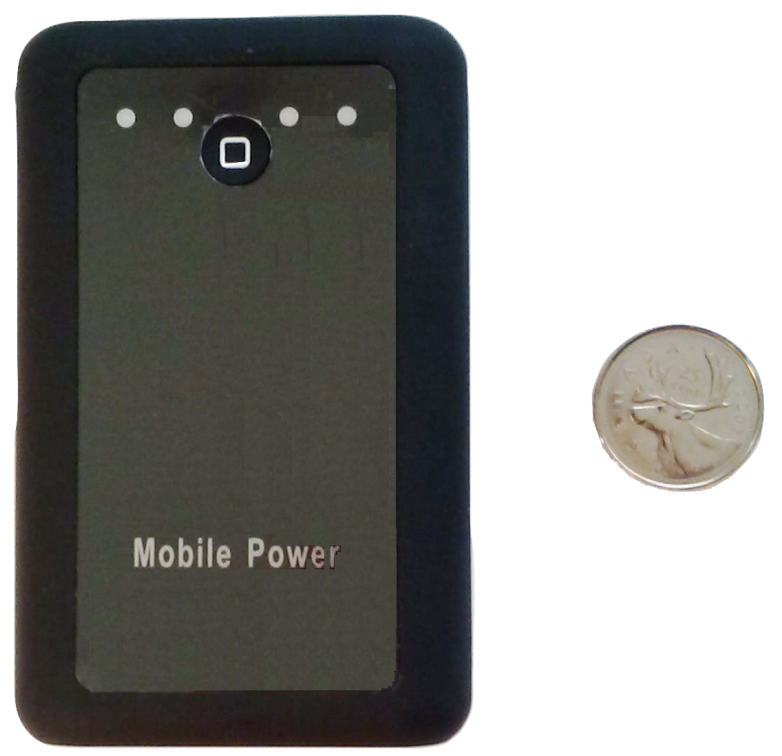

Figure 3.22: Battery pack size comparison with a quarter coin.

This type of battery packs are commonly used for charging portable devices such as cellphones, which require a $5 \mathrm{~V}$ voltage supply. In the case of the Raspberry $\mathrm{Pi}$, the battery is able to provide the $700 \mathrm{~mA}$ required for its operation. Estimating a max power consumption of $1 \mathrm{~A}$, the battery is able to provide power for about 6 hours.

The final cost of the system including the force sensors, electronic components, the Raspberry Pi and the ADC board was about US $\$ 360$. Table 3.9 shows the cost of the main components. Figure 3.23 shows the complete system mounted on a shoe. Upon system integration, calibration experiments will follow (as discussed in Chapter 4). 
Table 3.9: Estimated cost per insole.

\begin{tabular}{|c|c|c|c|}
\hline Component & Unit Price (US\$) & Quantity & Total (US\$) \\
\hline Flexiforce sensors & 14.63 & 14 & 204.75 \\
\hline Electronic components & 20.00 & & 20.00 \\
\hline Raspberry Pi & 35.00 & 1 & 35.00 \\
\hline ADC & 30.82 & 2 & 61.64 \\
\hline Liquid Rubber & 5.00 & & 5.00 \\
\hline Battery Pack & 33.28 & 1 & 33.28 \\
\hline Total & & & 359.67 \\
\hline
\end{tabular}

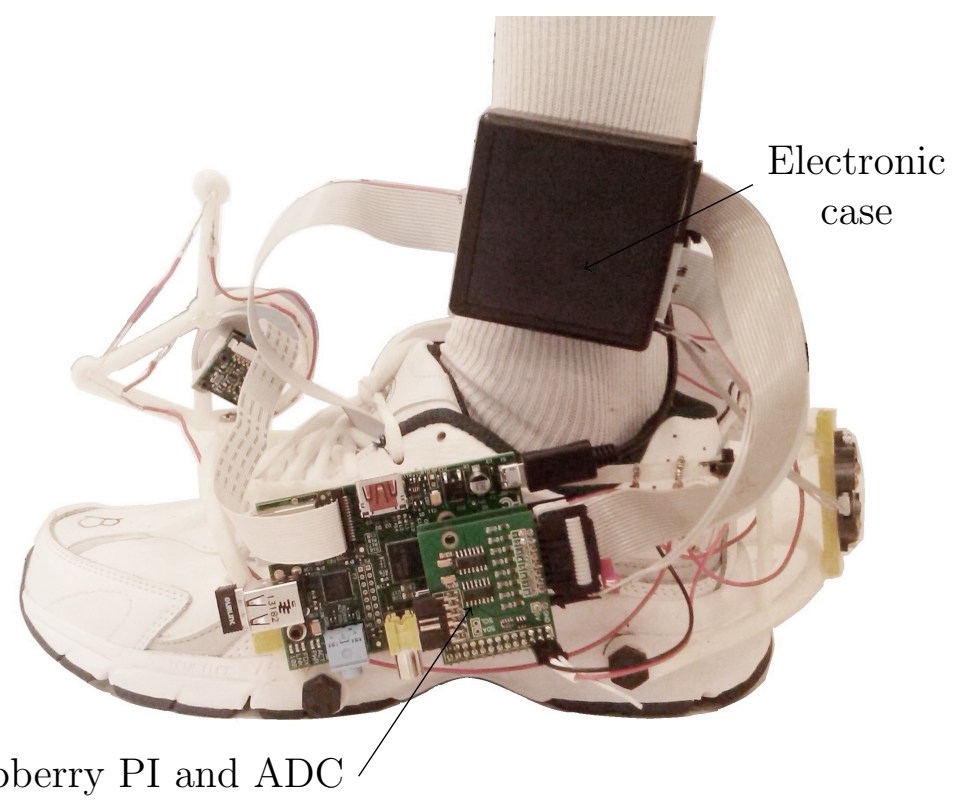

Figure 3.23: User wearing a shoe with the force sensing insole, the Raspberry Pi and the ADC mounted on it. The black case attached to the user ankle contains the electronics for the force sensors. 


\subsection{Summary}

This chapter presented the overall design of an insole capable of measuring the vertical ground reaction force without disturbing normal gait. Both a simulation of the forces under the foot from Section 3.3.5 and the experiments with the first prototype described in Section 4.2 were taken into account to decide the final number of sensors and the thickness of the insole. The final prototype is $8 \mathrm{~mm}$ thick and was built using 14 force sensors. The resulting insole is able to concentrate the forces on the contact areas of the sensors, while remaining thin and flexible to allow normal walking. Finally, the implementation with a real-time data acquisition system for the processing of the signals was presented. 


\section{Chapter 4}

\section{Evaluation of force sensing insole}

This chapter describes the calibration process of the force sensors used on the insole. Furthermore, it presents the experiments conducted with the first prototype of the insole that lead to changes and improvements on the design. Finally, a preliminary evaluation of the final insole is presented in both standing and walking conditions.

\subsection{Calibration}

In order to use the Flexiforce sensors for the measurement of the ground reaction forces a calibration procedure was needed to map the changes in the output of the sensors to the forces applied.

One important aspect to consider during this process is the way on which the sensors are loaded. Bending of the senors can bias the measurements and the readings may also differ when the application area of the force over the sensor changes. In order to investigate this last effect, a force sensor was loaded with the same weights but using small pieces of rigid material, or pucks, of different diameters as interface: 7, 8 and $9 \mathrm{~mm}$, all of which are smaller than the diameter of the active area of the sensor $(9.5 \mathrm{~mm})$. 


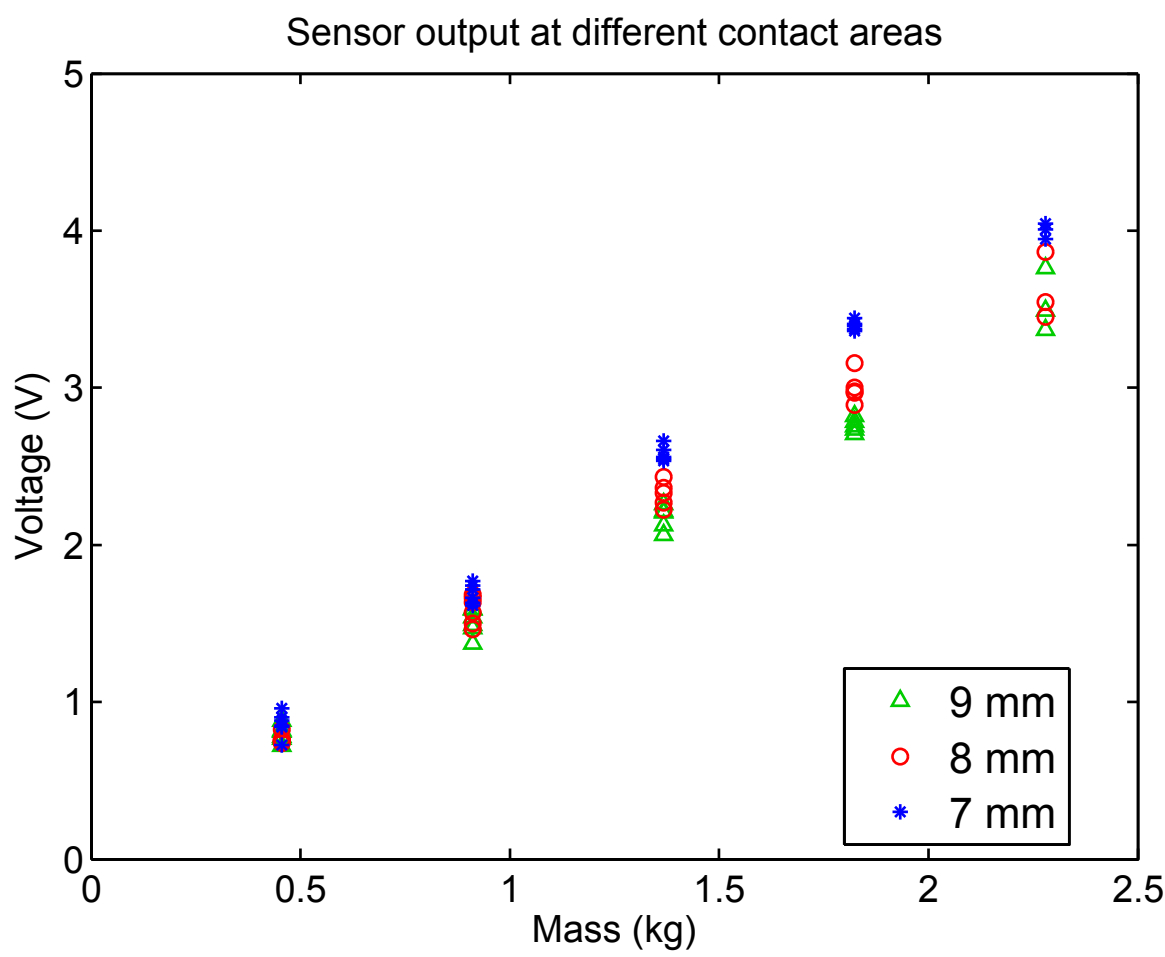

Figure 4.1: Differences in output voltage of same sensor when loaded with the same mass but with pucks of three different diameters. Active sensor area is $9.5 \mathrm{~mm}$.

From Figure 4.1 it can be observed that the change on the area of application of the force over the sensor has an effect on the output of the sensors, where as the contact area decreases, the output voltage of the sensor increases.

To investigate whether these variations are significant, a number of t-tests were performed. Because of the multiple comparisons, a correction factor was applied. Thus, the new significant value was $\alpha^{\prime}=\alpha / k=0.05 / 15=0.0033$. The results for an applied mass of $1.4 \mathrm{~kg}$ showed that there was a significant difference in the output for the $7 \mathrm{~mm}(M=2.58, S D=0.05)$ and $8 \mathrm{~mm}(M=2.33, S D=0.07)$ conditions; $t(9)=1.83, p<0.001$ and between the $7 \mathrm{~mm}(M=2.58, S D=0.05)$ and $9 \mathrm{~mm}(M=2.17, S D=0.08)$ conditions; $t(8)=1.94, p<0.001$. Similarly, for an applied mass of $1.8 \mathrm{~kg}$, there was significant difference in the output for the $7 \mathrm{~mm}(M=3.39, S D=0.04), 8 \mathrm{~mm}(M=3.01, S D=0.09)$ and $9 \mathrm{~mm}(M=$ 


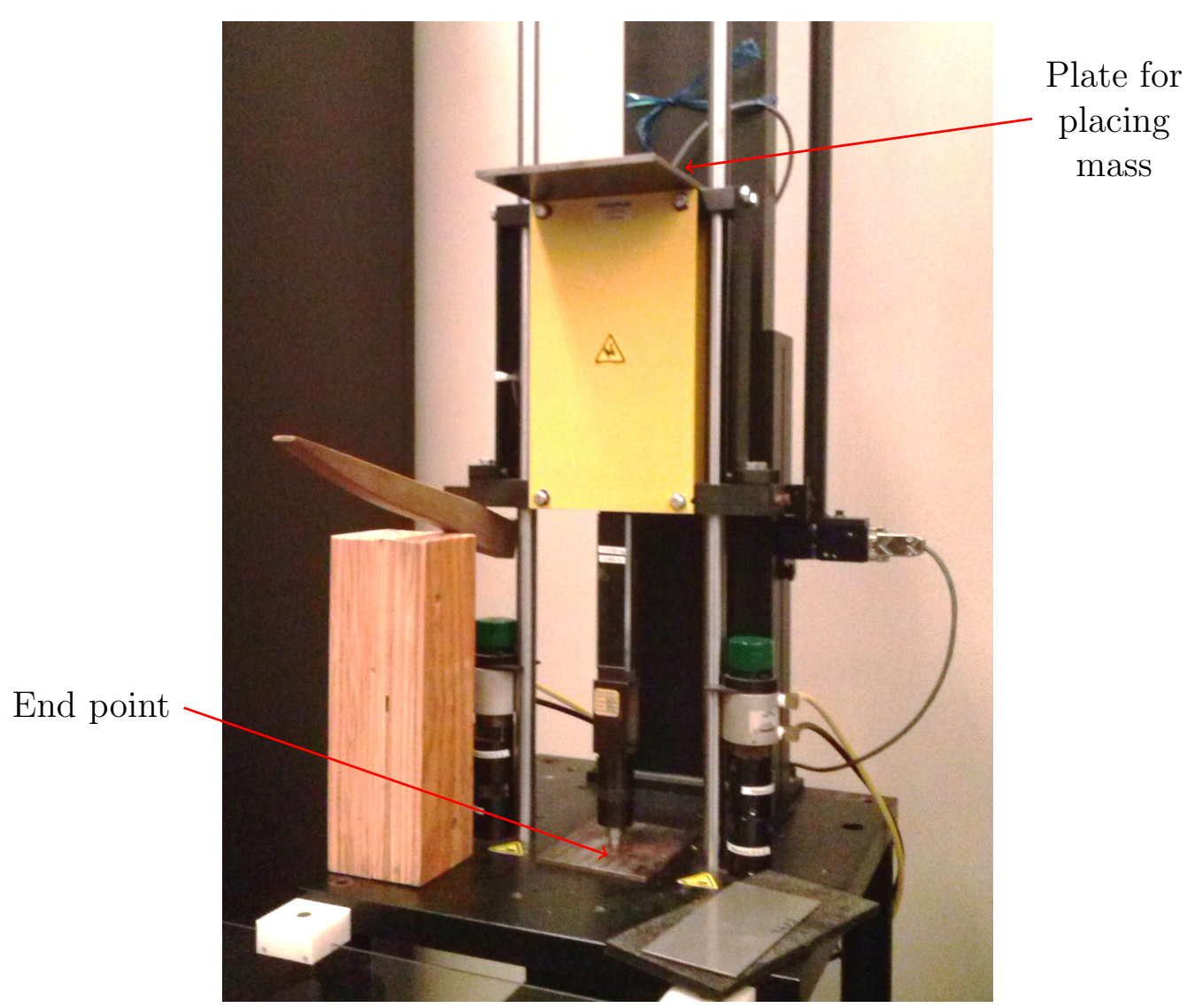

Figure 4.2: Set up for calibration of sensors using calibrated masses.

$2.76, S D=0.04)$ conditions; $t(7)=1.89, p<0.001$. This means that the output of the sensors is sensitive to the pressure applied over them and this should be taken into account during the calibration process. This is the main reason why the sensors were calibrated once they were attached between both layers in the rubber insole. This way the interface for the sensors during calibration is the same as the final application, thus increasing the reliability of the calibration process.

Calibration was performed individually for all 28 sensors to account for variability between them. Figure 4.2 shows the setup used for the calibration process. Calibrated masses were applied over each sensor through an end point of $9 \mathrm{~mm}$, while the signals from the sensor were recorded. The masses applied ranged from $2 \mathrm{~kg}$ up to $20 \mathrm{~kg}$. 


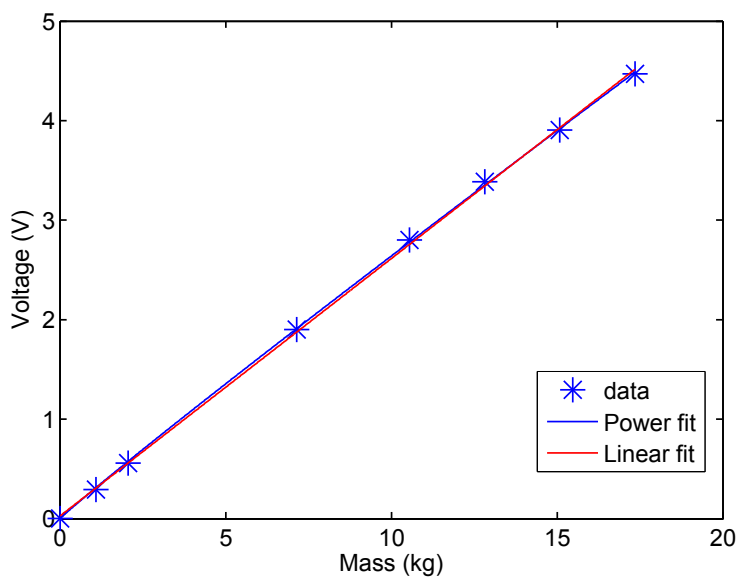

(a) Sensor 1 with linear curve fit

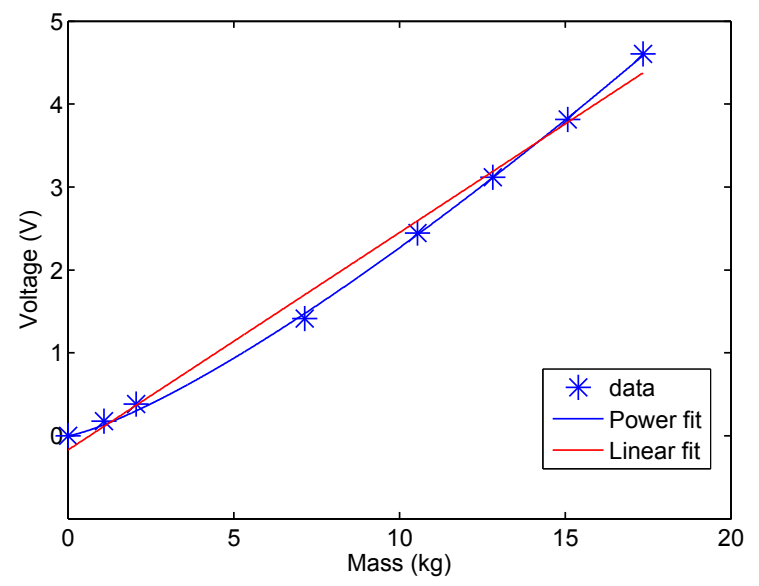

(b) Sensor 2 with power function curve fit

Figure 4.3: Linear and power curve fits for two different sensors. The blue points represents the measured points, the red line is the linear fit, and the blue line is the power function.

The data was then plotted and a curve was fit for each individual sensor. Despite the use of the inverse amplifier circuit, some of the sensors did not present a linear relationship as specified on the supplier's manual. Figure 4.3 shows an example of one of the sensors that presented a linear response and another with no linear response. A power function of the type

$$
F_{i}=a_{i} v_{i}^{b_{i}}
$$

was found to be the best fit, where $v_{i}$ is the output voltage of the sensor, $b_{i}$ and $a_{i}$ are the constants to be found, $F_{i}$ is the estimated force and $i$ is the number of sensor. Table 4.1 shows the calibration values obtained for each sensor on both insoles.

These calibration values were then implemented to map the relationship between the output voltage of the sensors and the forces applied in the calculation of the vertical ground reaction force (VGRF). 
The large variability in the $a_{i}$ and $b_{i}$ parameters in Table 4.1 shows that we need to have different calibration curves implemented in the sensors and average values are not usable.

Table 4.1: Calibration constants for the 14 sensor on both insoles.

\begin{tabular}{|c|c|c|c|c|}
\hline & \multicolumn{2}{|c|}{ Right } & \multicolumn{2}{c|}{ Left } \\
\hline & $a$ & $b$ & $a$ & $b$ \\
\hline Sensor 1 & 5.91 & 0.85 & 6.33 & 1.07 \\
\hline Sensor 2 & 10.69 & 0.70 & 10.29 & 0.90 \\
\hline Sensor 3 & 8.34 & 0.73 & 9.70 & 0.88 \\
\hline Sensor 4 & 9.44 & 0.68 & 3.88 & 1.03 \\
\hline Sensor 5 & 9.75 & 0.69 & 3.70 & 1.00 \\
\hline Sensor 6 & 8.00 & 0.69 & 7.80 & 1.04 \\
\hline Sensor 7 & 6.11 & 0.77 & 4.42 & 0.97 \\
\hline Sensor 8 & 6.28 & 0.83 & 5.18 & 0.80 \\
\hline Sensor 9 & 5.92 & 0.76 & 3.63 & 1.06 \\
\hline Sensor 10 & 10.74 & 0.71 & 7.21 & 1.09 \\
\hline Sensor 11 & 10.16 & 0.72 & 4.60 & 0.99 \\
\hline Sensor 12 & 8.32 & 0.69 & 3.64 & 1.04 \\
\hline Sensor 13 & 7.14 & 0.78 & 3.94 & 1.00 \\
\hline Sensor 14 & 5.45 & 0.95 & 7.24 & 1.08 \\
\hline
\end{tabular}

\section{$4.2 \quad$ First prototype experiments}

As mentioned in Chapter 3 the first prototype of the insole was made using 12 force sensors. Two types of experiments were carried out to evaluate the performance of the insole for measurement of the normal component of the ground reaction force and center of pressure: standing and walking experiments. One female individual, weight 


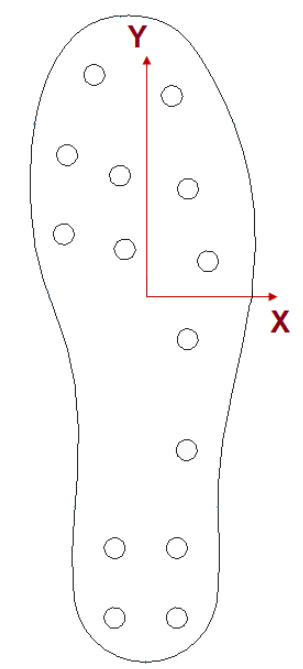

Figure 4.4: Coordinate system on the insole.

$=60 \mathrm{~kg}$, shoe size $=6$ (US) participated in these verification tests. The normal ground reaction force was calculated by summing the forces measured by each sensor and the COP along the $\mathrm{x}$ and $\mathrm{y}$ axes were calculated as the weighted mean of the position of the sensors, using forces as the weights on each sensor, as follows:

$$
\begin{aligned}
& C O P_{x}=\frac{\sum_{i=1}^{n} F_{i} x_{i}}{\sum_{i=1}^{n} F_{i}}, \\
& C O P_{y}=\frac{\sum_{i=1}^{n} F_{i} y_{i}}{\sum_{i=1}^{n} F_{i}}
\end{aligned}
$$

where $F_{i}$ is the force on the $i$ sensor, $x_{i}$ and $y_{i}$ are the positions of the sensor on the medial/lateral and anterior/posterior directions and $n$ is the total number of sensors. Figure 4.4 shows the origin of the $\mathrm{x}$ and $\mathrm{y}$ axes of the insole.

\subsubsection{Standing experiments}

The objective of these experiments was the static evaluation of the insole to measure the vertical component of the GRF. For the standing experiments, the insole was 
taped to a Bertec force plate and one subject stood on the insole for about 10 seconds. The forces from the insole and the force plate were both recorded. Figure 4.5 shows a comparison of both measurements while a subject stood on one foot (single stance). Figure 4.6 shows the recording from one foot, but this time the subject was standing on both feet (double stance), with only one foot over the force plate.

The double stance condition showed good correspondence between the insole measurement and the force plate, with measured values of $302 \mathrm{~N}$ and $290 \mathrm{~N}$ respectively. In the case of single stance, the force recorded by the insole was $540 \mathrm{~N}$ while the force plate was $599 \mathrm{~N}$ which represent an error of $59 \mathrm{~N}(9.85 \%)$.

Next, the insole was placed inside a pair of shoes and the same standing tests were repeated. Figure 4.7 and 4.8 show the results from these tests while a subject was standing on one and two feet respectively. For the double stance condition, the values measured by the insole and force plate were 307 and $316 \mathrm{~N}$. In the case of single stance, the force recorded by the insole was $481 \mathrm{~N}$ while the force plate was $601 \mathrm{~N}$ which represent an error of $120 \mathrm{~N}(20.0 \%)$.

The difference in values from the condition of the insole alone and on the shoe can be caused by the softer surfaces inside the shoe, which allows for indentation and deflections, as well as some angled sensor surfaces.

One of the issues that was identified during these static tests was that the force measurement dropped compared to the force plate when the subject shifted the body weight toward the back. Figure 4.9 illustrates this problem when at around 17 and 24 sec of the recording there is a drop on the value of the force measured by the insole when the $C O P_{y}$ has moved toward the back of the foot. For this reason an extra sensor was added to the heel area of the insole, to improve the system. 


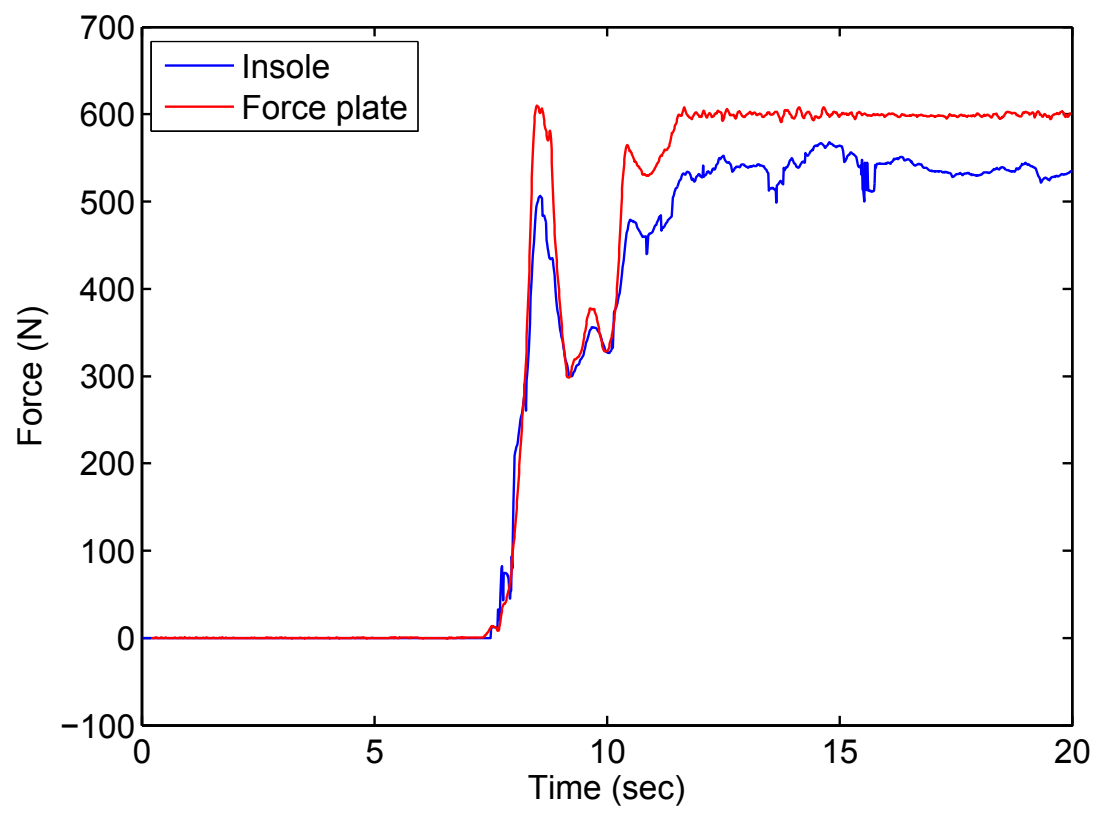

Figure 4.5: Static measurement of prototype 1 of the insole compared to a force plate for a subject during single stance.

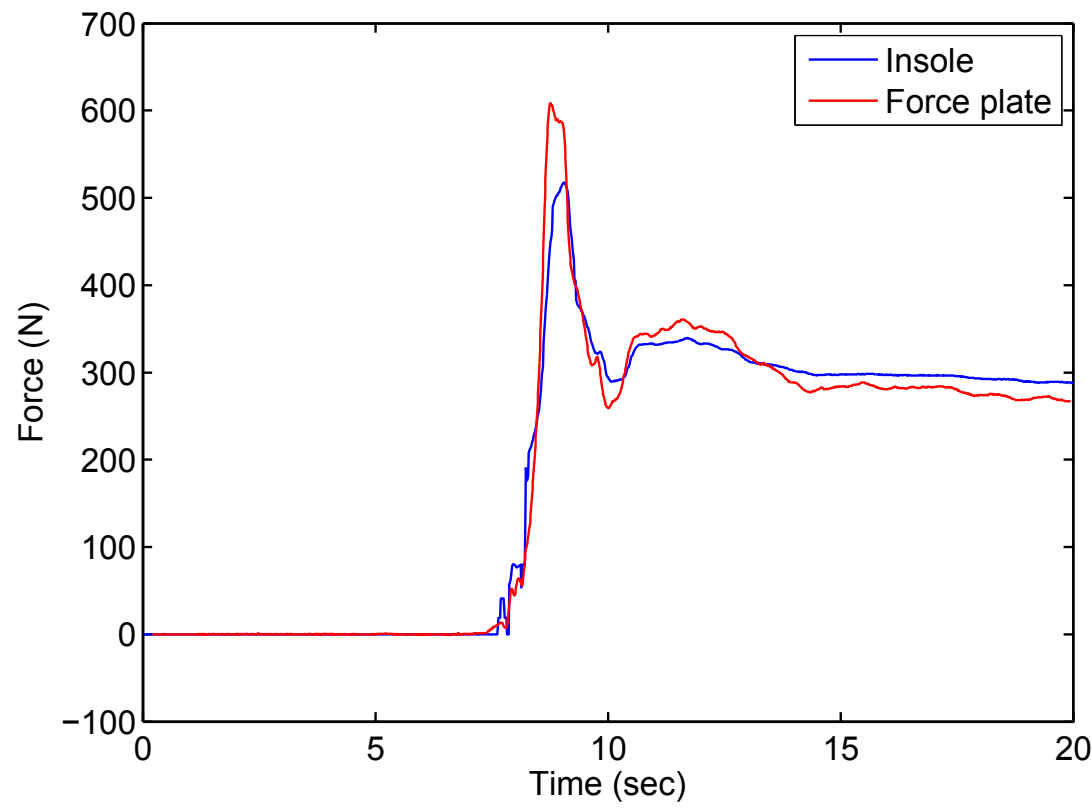

Figure 4.6: Static measurement of prototype 1 of the insole compared to a force plate for a subject during double stance. 


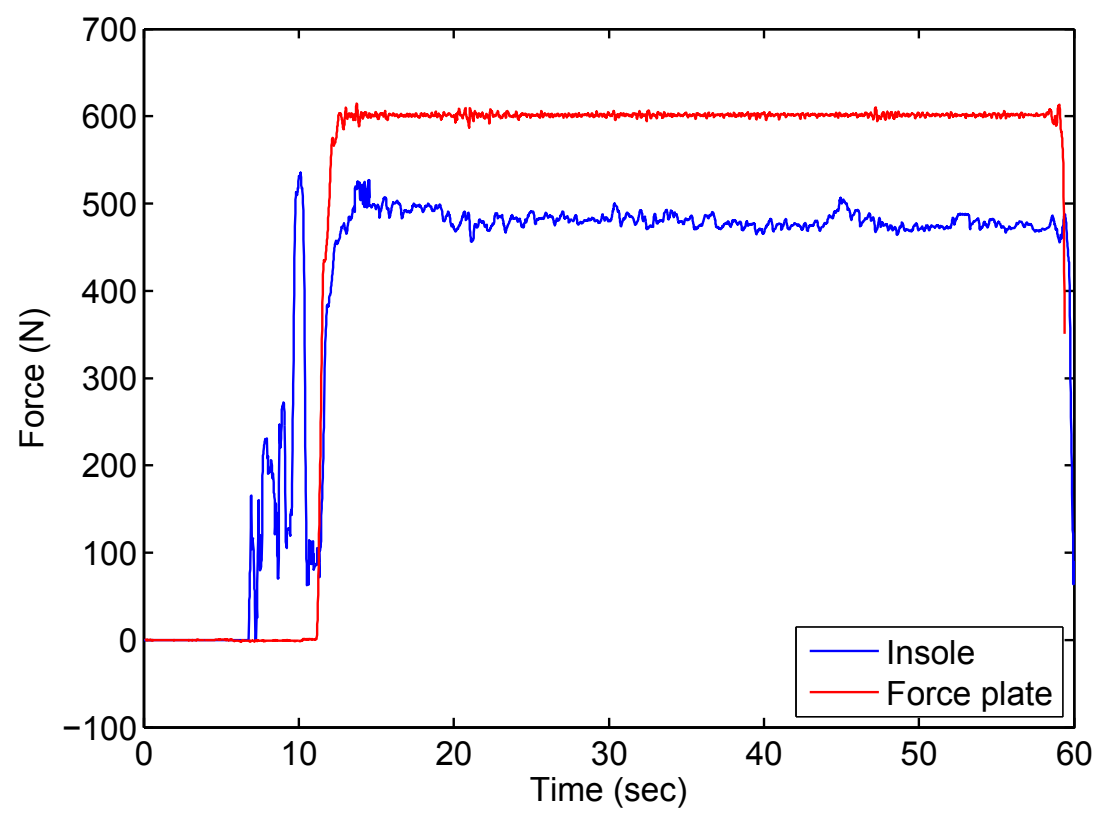

Figure 4.7: Static measurement of prototype 1 inside the shoe compared to a force plate for a subject during single stance.

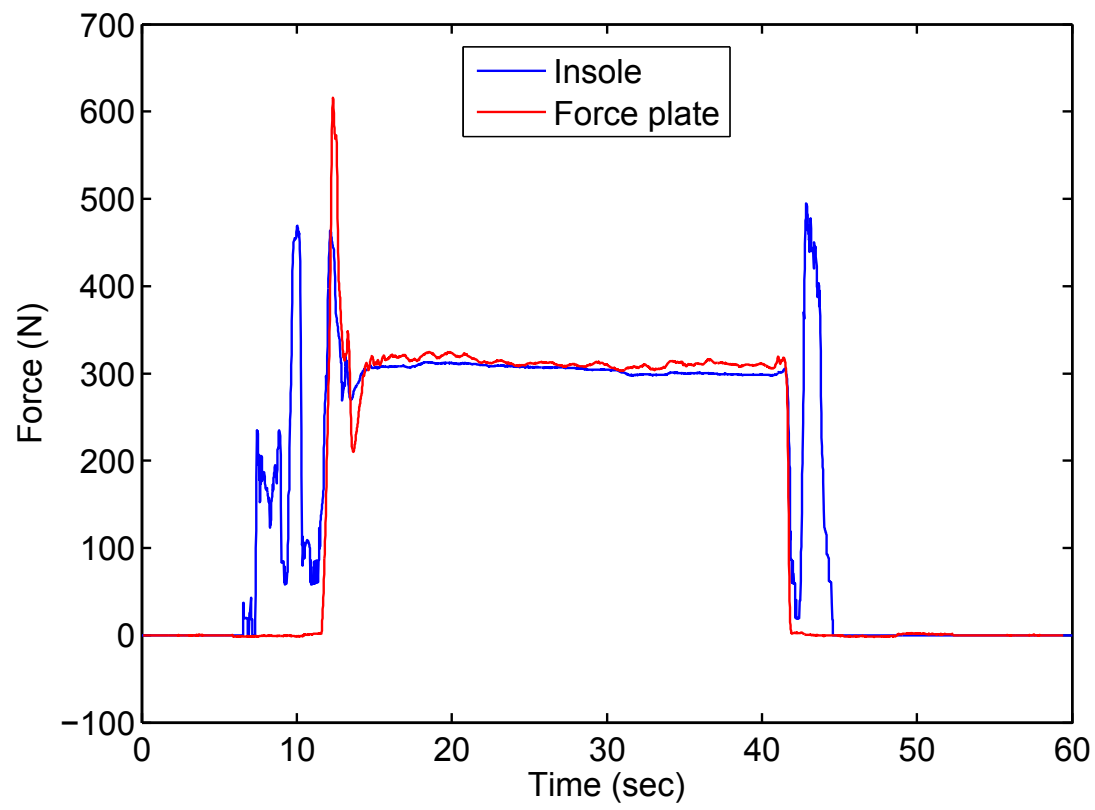

Figure 4.8: Static measurement of prototype 1 inside the shoe compared to a force plate for a subject during double stance. 

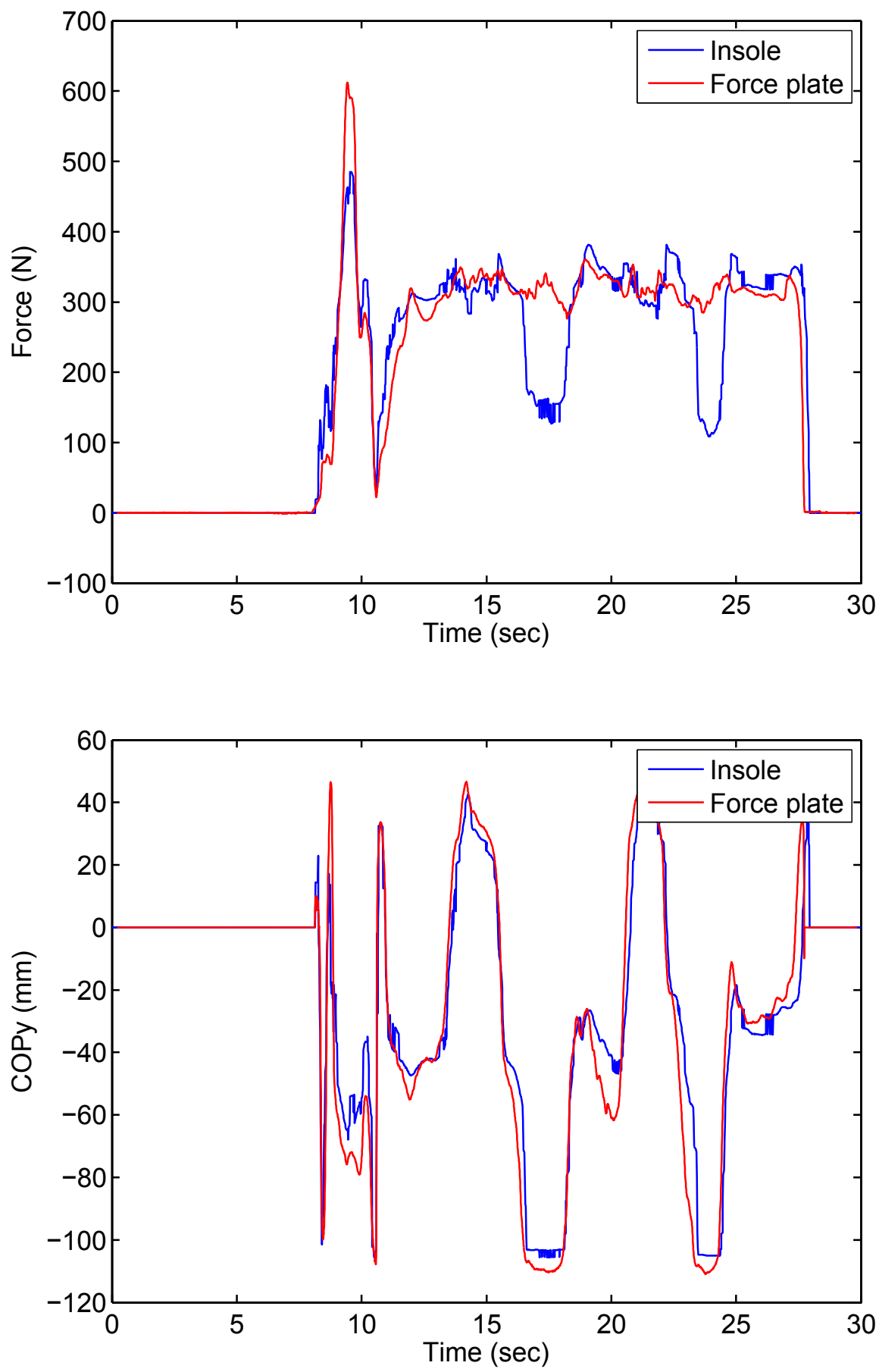

Figure 4.9: Static measurement of prototype 1 of the insole compared to a force plate for a subject during double stance leaning forth and back showing the decrease of force when the weight is shifted to the back of the foot. 


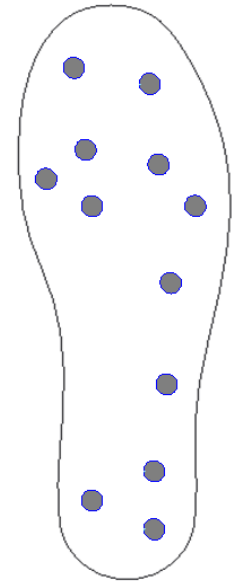

(a) Prototype 1

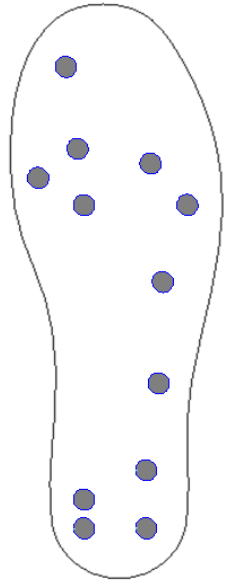

(b) Prototype 2

Figure 4.10: (a) First prototype of the insole. (b) Modified prototype of the insole with the position of one sensor changed from the toes to the heel.

A modified version of the insole (Prototype 2) was made by moving the sensor on the toes to the heel as shown in Figure 4.10

Prototype 2 was again tested by standing on two feet and leaning forth and back. This time the force values recorded by the insole did not present the big drops when the weight was shifted to the back as can be seen on Figure 4.11 . Therefore, for the final design on the insole a total of 4 sensors were placed on the heel instead of the original 3.

\subsubsection{Walking experiments}

Once the first prototype of the insole was modified, walking experiments were carried out with prototype 2 to investigate the dynamic performance of the insole. Again, the insole was taped to a Bertec force plate and one subject stepped on the insole lining up the foot as best as he could. Figure 4.12 shows the vertical ground reaction force (VGRF), the COP on the insole coordinate system, the COP in the medial/lateral 

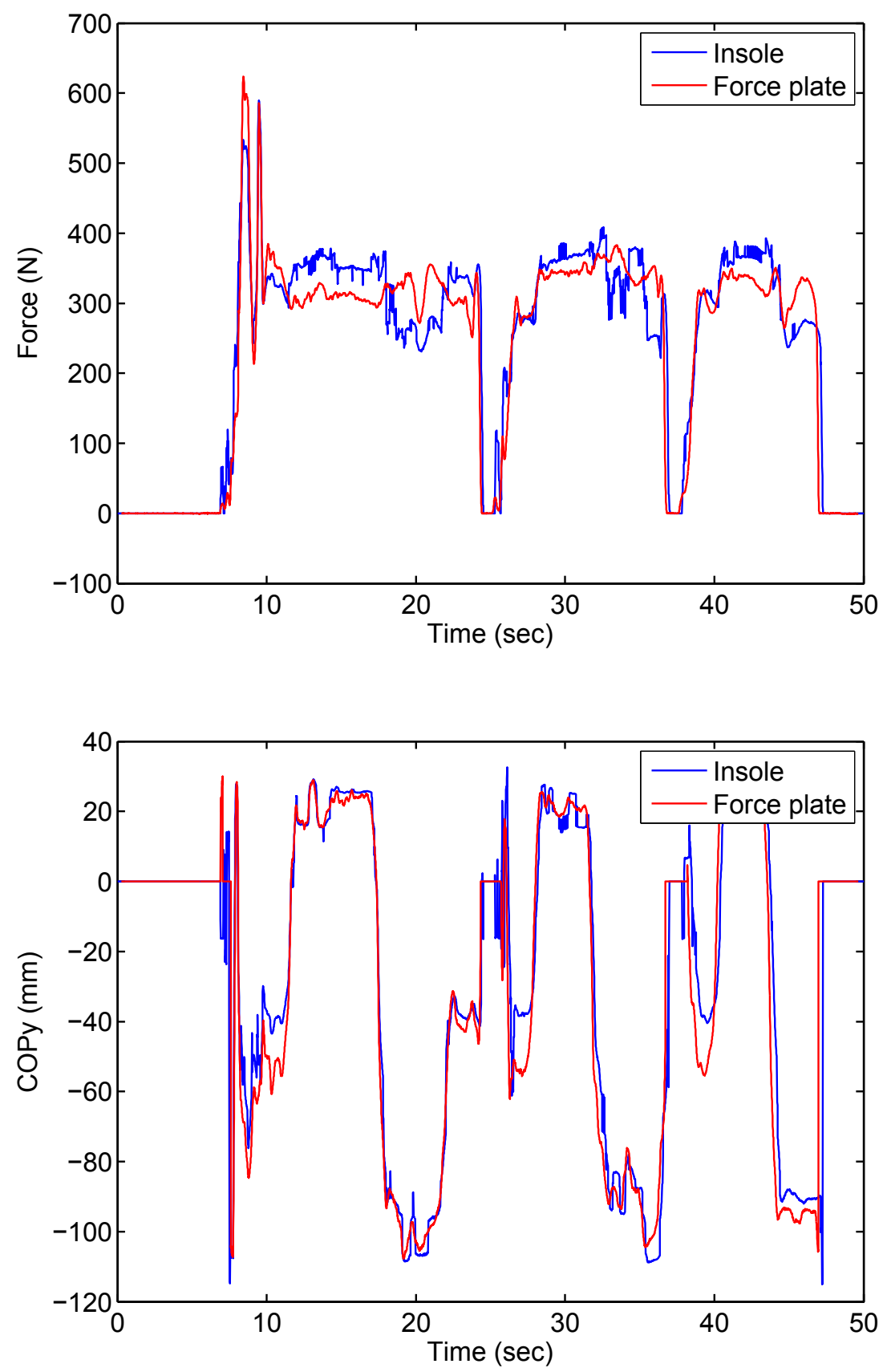

Figure 4.11: Static measurement of prototype 2 of the insole compared to a force plate for a subject during double stance leaning forth and back after adding an extra sensor on the heel. 
direction and the $\mathrm{COP}$ in the anterior/posterior direction as a function of time for one example trial compared to the measurements of the force plate.

The correlation values and root mean square errors (RMSE) for 10 steps are shown on Tables 4.3 and 4.2 . The high correlation values for the VGRF and COPy (above 0.95) show the good correspondence between the measurements of the insole and the values recorded by the force plate. In the case of the $\mathrm{COPx}$, the mean correlation value was 0.72 , and the mean RMSE was $5.46 \mathrm{~mm}$. Furthermore, from Figure 4.12 it can be observed how the shapes of the VGRF and COP from the insole follow the same trend as the force plate measurements. These results show the capability of the insole to measure the GRF and COP.

Table 4.2: Correlation and RMS errors of VGRF for prototype 2 of the insole for 10 steps of the walking experiments.

\begin{tabular}{|c|c|c|c|}
\hline & \multicolumn{3}{|c|}{ VGRF } \\
\hline & Corr & RMSE (N) & \%RMSE \\
\hline Mean & $0.97(0.01)$ & $45.94(15.60)$ & $7.00(2.38)$ \\
\hline Max & 0.99 & 82.56 & 12.59 \\
\hline Min & 0.98 & 26.93 & 4.08 \\
\hline
\end{tabular}

Table 4.3: Correlation and RMS errors of COP prototype 2 of the insole for 10 steps of the walking experiments.

\begin{tabular}{|c|c|c|c|c|c|c|}
\hline & \multicolumn{3}{|c|}{ COPx } & \multicolumn{3}{c|}{ COPy } \\
\hline & Corr & RMSE $(\mathrm{mm})$ & \%RMSE & Corr & RMSE $(\mathrm{mm})$ & \%RMSE \\
\hline Mean & $0.72(0.13)$ & $5.46(1.91)$ & $12.29(3.17)$ & $0.99(0.01)$ & $8.08(3.74)$ & $4.85(2.35)$ \\
\hline Max & 0.94 & 7.96 & 16.04 & 0.99 & 14.76 & 8.43 \\
\hline Min & 0.56 & 3.26 & 7.71 & 0.97 & 4.04 & 2.27 \\
\hline
\end{tabular}




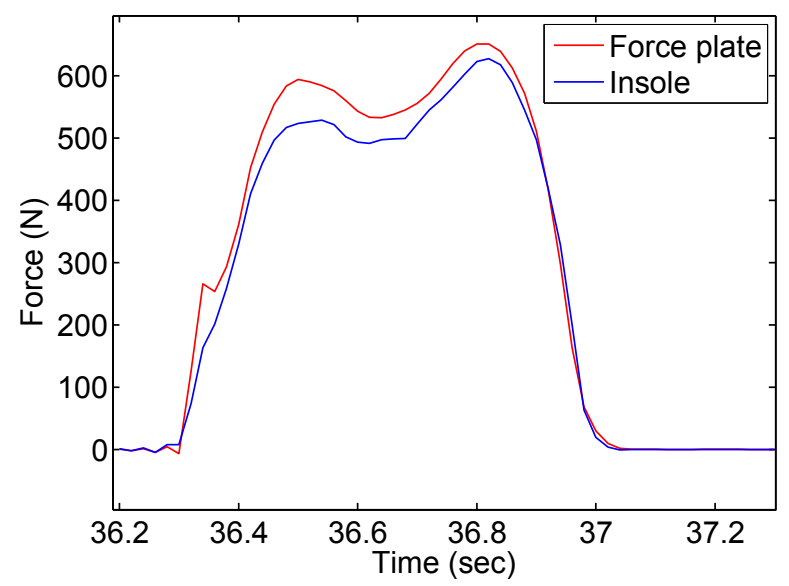

(a) VGRF

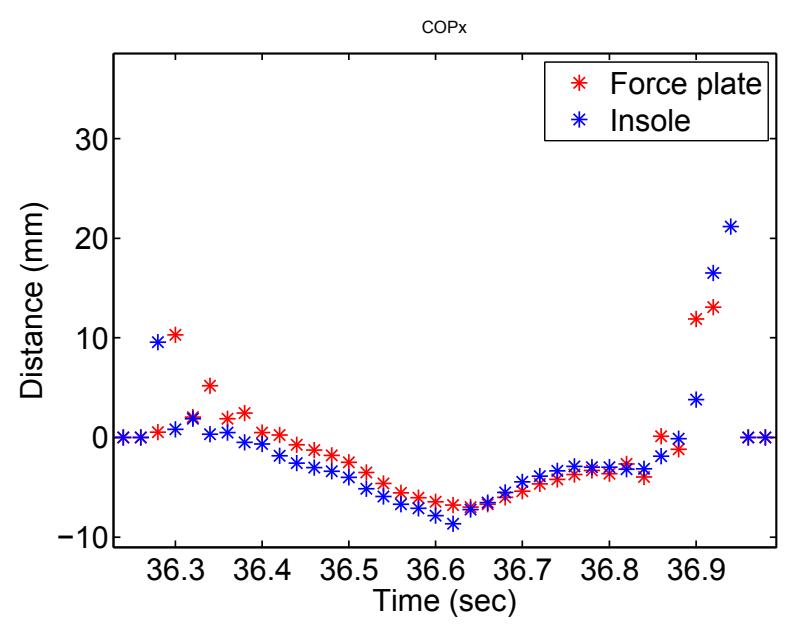

(c) $\mathrm{COPx}$

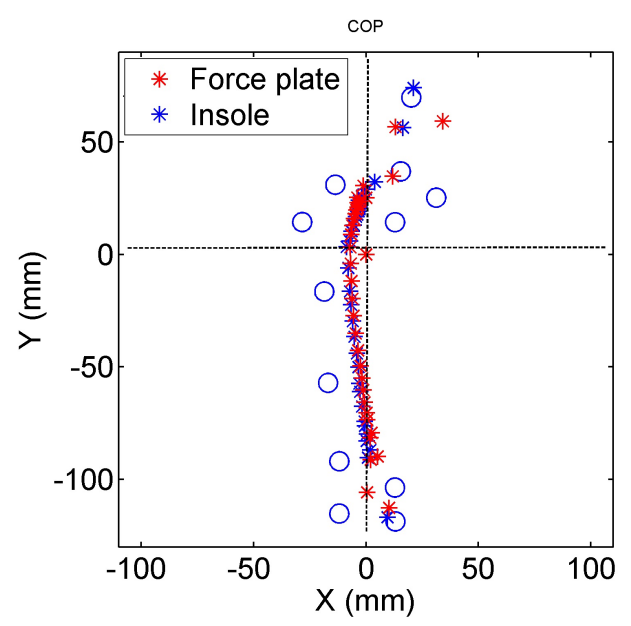

(b) COP

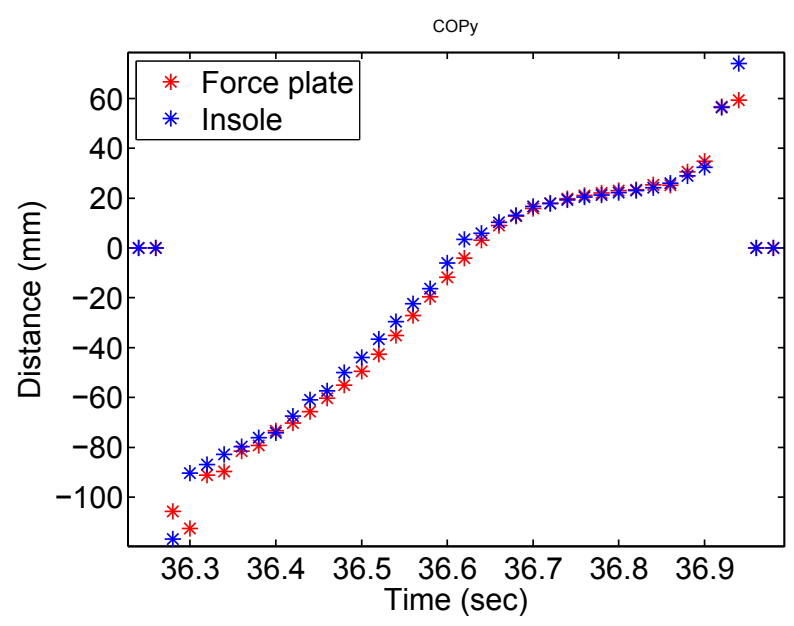

(d) $\mathrm{COPy}$

Figure 4.12: Comparison of prototype 2 of the insole (blue) and force plate (red) measurements of GRF and COP for one example step of the left foot during the walking experiments. 


\subsection{Preliminary experiments with final insole}

The final prototype of the insole, prototype 3, was built using 14 force sensors as described on Section 3.3.6 The objectives of these experiments were to perform a preliminary evaluation of the final design to measure the VGRF and COP during standing and walking.

\subsubsection{Standing experiments}

The same procedure as Section 4.2 was carried out for the standing experiments. The prototype 3 was taped to a Bertec force plate and one subject stood on the insole for about 10 seconds. Both the forces from the insole and the force plate were recorded. Figure 4.13 shows a comparison of both measurements for a subject during single stance and Figure 4.14 shows also a recording from one foot, during double stance.

The double stance condition showed good correspondence between the insole measurement and the force plate, with measured values of $249 \mathrm{~N}$ and $270 \mathrm{~N}$ respectively. In the case of single stance, the force recorded by the insole was $526 \mathrm{~N}$ while the force plate was $589 \mathrm{~N}$ which represent an error of $63 \mathrm{~N}$ (10.7\%). From Figure 4.14 it can be observed that as the insole is constantly loaded over time, the value of the forces decreases slightly, this is caused by the drift of the sensors. Therefore, the insole shows a better performance during dynamic loading compared to static situations.

An additional experiment was carried out while the subject was standing on two legs with one foot over the insole and force plate. This time the subject shifted his weight forth and back to check the measurements of the insole in different conditions. Figure 4.15 (a) shows the output obtained from the insole compared to the force values measured by the force plate and (b) shows the recording of the COP in the anterior/posterior direction. This time there were no major drops on the force 
values from the insole. Furthermore there was a good correspondence between COPy measurements of the insole and the force plate.

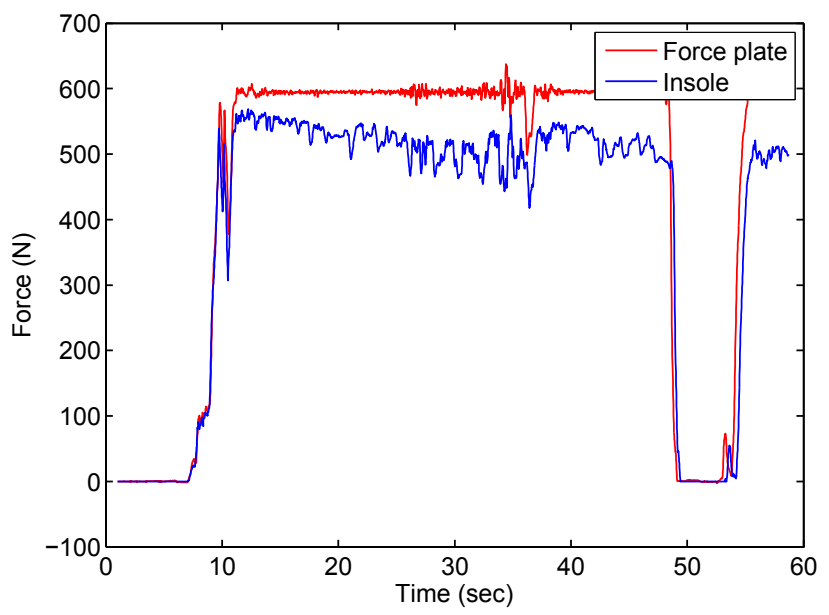

Figure 4.13: Static measurement of prototype 3 of the insole compared to a force plate for a subject during single stance.

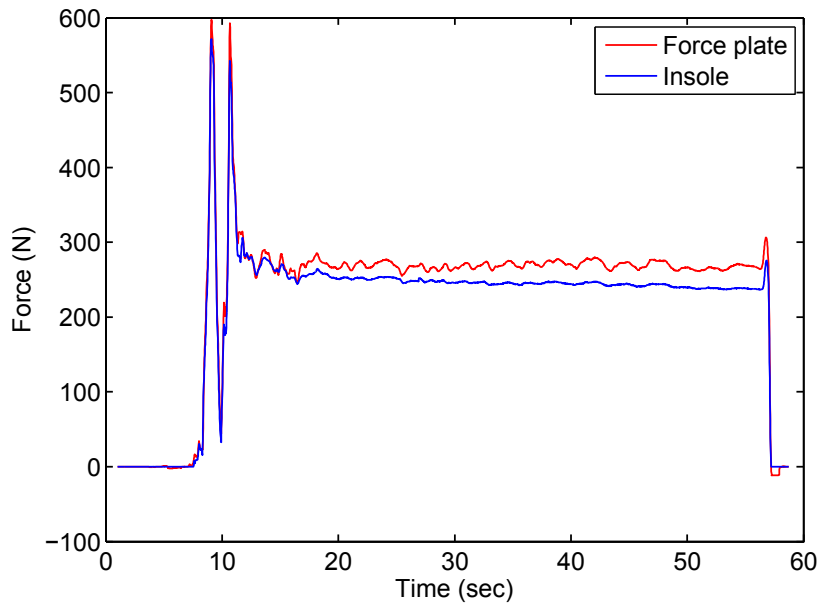

Figure 4.14: Static measurement of prototype 3 of the insole from one leg compared to a force plate for a subject during double stance. 

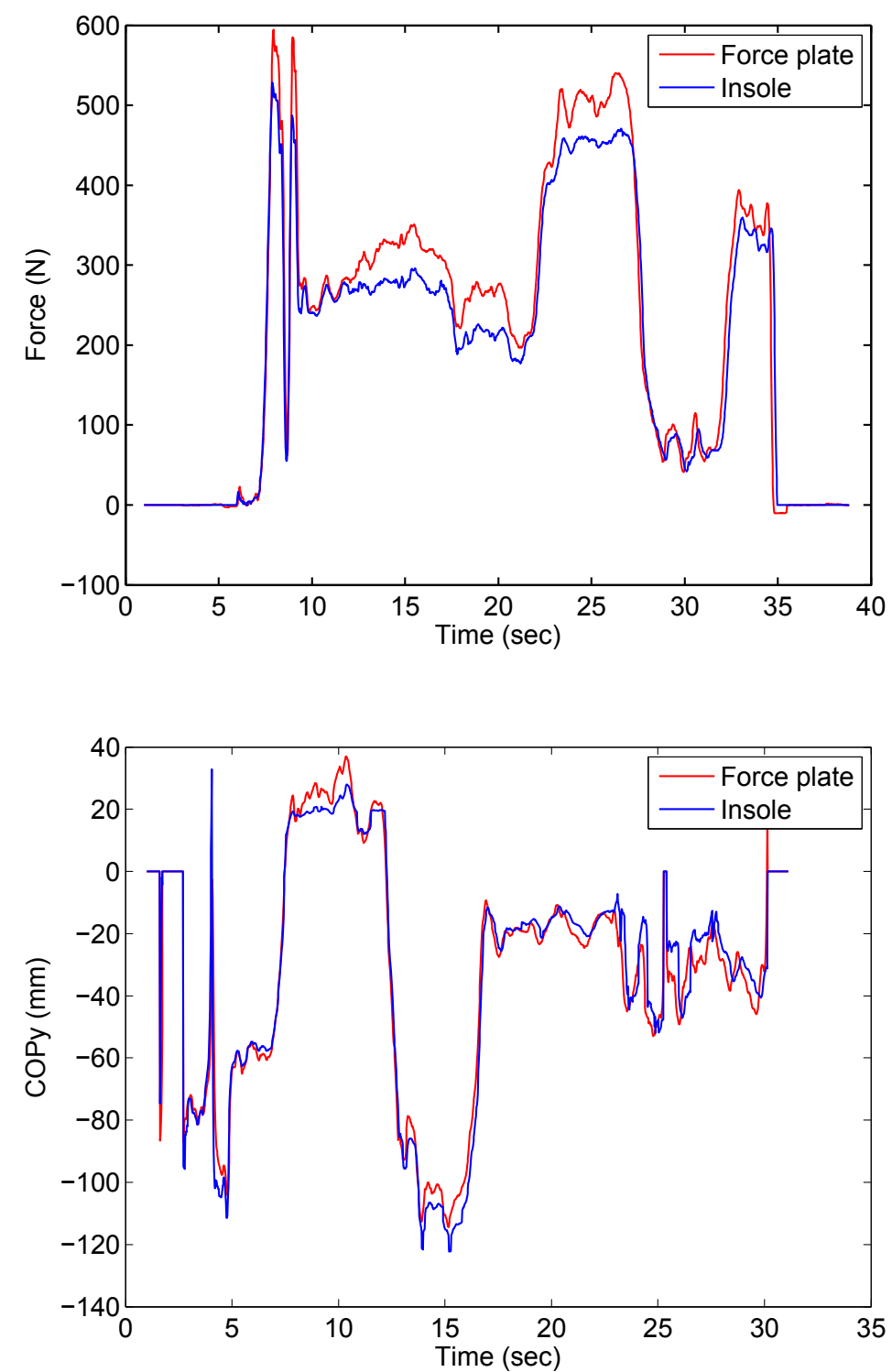

Figure 4.15: Static measurement of prototype 3 of the insole compared to a force plate for a subject during double stance leaning forth and back. 


\subsubsection{Walking experiments}

Finally, dynamic experiments were carried out with the final insole. Again, the insole was taped to a Bertec force plate and a person stepped on the insole lining up the foot as best as he could. Figure 4.16 show the vertical ground reaction force, the $\mathrm{COP}$ on the insole coordinate system, the $\mathrm{COP}$ in the medial/lateral direction and the COP in the anterior/posterior direction as a function of time for one example trial compared to the measurements of the force plate. The correlation values and RMSE for 12 steps are shown on Table 4.4. When compared with the values obtained on Section 4.2, it can be seen that there were improvements on the correlations and RMSE recorded for the final insole.

Table 4.4: Correlation and RMS errors for prototype 3 of the insole for the walking experiments

\begin{tabular}{|c|c|c|c|c|c|c|}
\hline & \multicolumn{3}{|c|}{ Correlation } & \multicolumn{3}{c|}{ RMSE } \\
\hline & VGRF & COPx & COPy & VGRF (N) & COPx (mm) & COPy $(m m)$ \\
\hline Mean & $0.97(0.01)$ & $0.84(0.14)$ & $0.99(0.01)$ & $46.16(11.17)$ & $2.72(1.26)$ & $3.50(1.05)$ \\
\hline Max & 0.98 & 0.98 & 0.99 & 61.53 & 4.88 & 5.47 \\
\hline Min & 0.95 & 0.60 & 0.98 & 30.15 & 1.01 & 1.77 \\
\hline
\end{tabular}




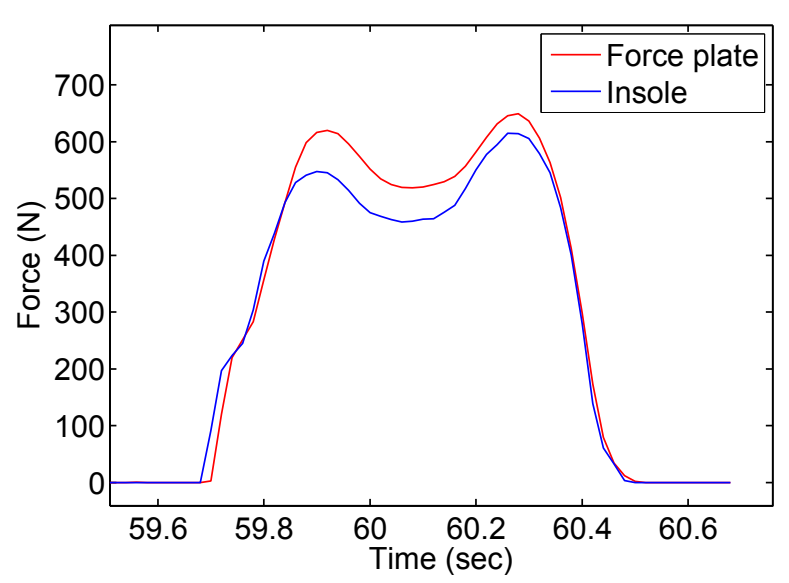

(a) VGRF

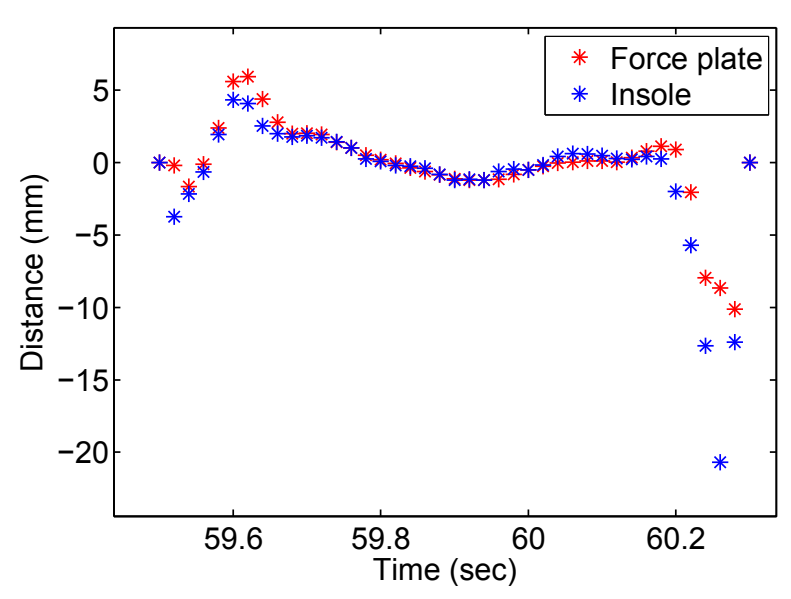

(c) $\mathrm{COPx}$

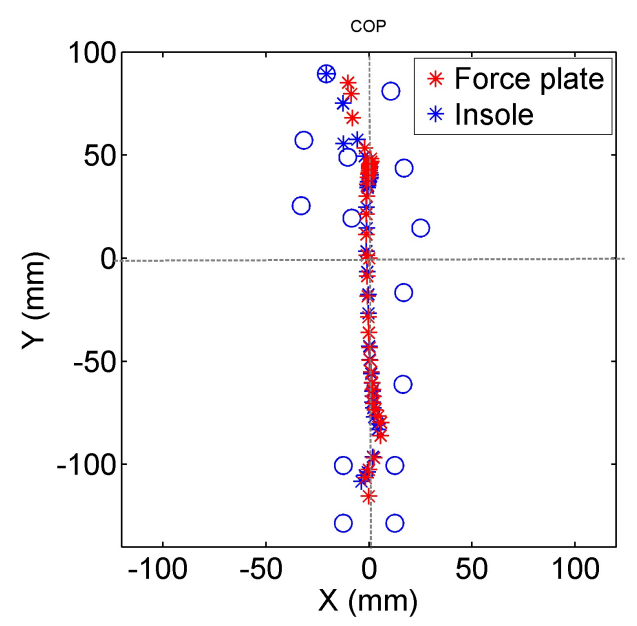

(b) COP

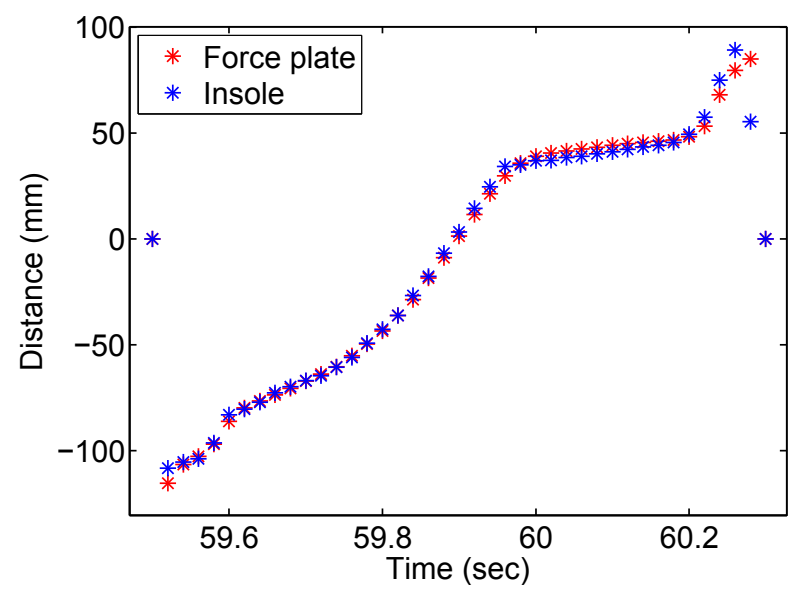

(d) $\mathrm{COPy}$

Figure 4.16: Comparison of prototype 3 of the insole (blue) and force plate (red) measurements of GRF and COP for one example step of the walking experiments 


\subsection{Summary}

This chapter presented the calibration process used for the force sensors and the preliminary experiments of a first prototype that lead to improvements on the design for the final insole.

Preliminary experiments showed better results when the insole was tested against a rigid surface such as the floor (9.85\% of error), compared to the results obtained once it was placed inside the shoe (20.00\% of error) during single stance static experiments. This can be due to the more compliant materials inside the shoe, which allows for indentation and deflections, as well as some angled sensor surfaces. On the other hand, better results were obtained during dynamic testing (walking experiments) compared with static test, with errors of $7.85 \%$ and $10.70 \%$ for the dynamic and static experiments respectively. Table 4.5 shows a comparison between the prototypes in the static and dynamic experiments.

Table 4.5: Comparison of correlations (R) and RMSE from the insole prototypes.

\begin{tabular}{|c|c|c|c|c|}
\hline \multicolumn{5}{|c|}{ Static Tests } \\
\hline & \multicolumn{2}{|c|}{ Prototype 1} & \multicolumn{2}{|c|}{ Prototype 3} \\
\hline & \multicolumn{2}{|r|}{ RMSE } & \multicolumn{2}{|r|}{ RMSE } \\
\hline Single stance & \multicolumn{2}{|r|}{$9.85 \%$} & \multicolumn{2}{|r|}{$10.70 \%$} \\
\hline Double stance & \multicolumn{2}{|r|}{$4.26 \%$} & \multicolumn{2}{|r|}{$7.66 \%$} \\
\hline \multicolumn{5}{|c|}{ Walking Tests } \\
\hline & \multicolumn{2}{|c|}{ Prototype 2} & \multicolumn{2}{|c|}{ Prototype 3} \\
\hline & $\mathbf{R}$ & RMSE & $\mathbf{R}$ & RMSE \\
\hline Walking VGRF & 0.97 & $7.81 \%$ & 0.97 & $7.85 \%$ \\
\hline Walking COPx & 0.72 & 5.46 & 0.84 & 2.72 \\
\hline Walking COPy & 0.99 & 8.08 & 0.99 & 3.50 \\
\hline
\end{tabular}




\section{Chapter 5}

\section{Shear forces estimation}

As explained in Section 2.2.1 the ground reaction force is composed of three com-

ponents, the normal, the anterior/posterior and medial/lateral forces. In Section 4.3 we showed the resulting measurements from the force sensing insole for the vertical ground reaction force and the center of pressure, which can be used for the assessment of balance. However, the shear components of the ground reaction force provide also useful information about gait and balance which could enable more accurate balance measures. This chapter proposes a methodology for estimating the shear components of the ground reaction force during walking using the force sensing insole previously developed with the help of artificial neural networks.

\subsection{Previous attempts at estimating shear compo- nents of the ground reaction force}

As mentioned in Chapter 2, there have been several attempts to develop mobile devices to measure the complete ground reactions forces. However these devices have the limitation of being too rigid and increasing the height in the outsole, modifying the normal gait patterns and the original interface between shoe and ground. 
Some attempts have been previously made to estimate the shear components of the ground reaction force using the information from commercial pressure insoles, which only measure the normal component of the GRF.

In [54 an artificial neural network was used as a learning technique for estimation of the horizontal fore-aft component of the GRF. However they did not estimate the medial/lateral component of the GRF. Later in [55], a method was presented for calculating the complete ground reaction forces from pressure insoles using kinematic information from a motion capture system. Despite the good results obtained, the technique remains limited to laboratory environment due to the use of the camera for the estimation. Another two studies [56], 57] made use of pressure insole and learning techniques such as linear regression and artificial neural networks for estimation of the shear components of the GRF. However, because of the large number of sensors present on the pressure insole, they had to reduce the number of input variables used on the estimation. The current findings are limited to a small homogenous group of male young subjects only. Future studies should aim to generalize the methods, or to establish specific methods for other subjects, patients, motions, footwear and floor conditions

All this previous studies showed the possibility of predicting the shear components of the GRF from pressure data since there seems to be a relation between the distribution of the normal force on the foot and the shear ground reaction forces, however this has not been described based on any proper mechanical model. Moreover, they all made use of commercial pressure insole with sensors placed all over the surface of the foot. Therefore, the goal is to investigate if our system, with fewer sensors, is capable of predicting the shear components.

Since we want to use the 14 sensors from the insole as inputs for a nonlinear model in which the relationship is not analytically available, a computational learning technique such as artificial neural networks may provide a solution for finding such a 
model. The next sections describe the methodology used for estimation of the shear components of the GRF using the force sensing insole and artificial neural networks.

\subsection{Artificial Neural Networks}

Artificial Neural Networks (ANN) are a machine learning technique that is inspired by how neurons in the brain interact to make decisions and perform tasks. The underlying idea is to mimic their ability to learn from previous examples and process data in parallel instead of a linear fashion. However, it can be simply assumed as a computational method which aims at optimizing a function of interest. It uses known inputs and outputs to tune its internal coefficients through an optimization process. Then, the model produced can be used to estimate the outputs of a system from new data. ANN are capable of solving nonlinear problems where a model of the process is not available, known as black box.

ANN are composed of a series of interconnected units called neurons. A schematic of a single neuron is shown in Figure 5.1. Each neuron has an input/output (I/O) characteristic and implements a local computation or function. The output of the neuron is a nonlinear combination of the inputs $x_{i}$, weighted by the parameters $\omega_{i}$, with an additional constant term called bias, $\omega_{o}$ such that:

$$
y=f\left(\omega_{o}+\sum_{i=1}^{n} \omega_{i} x_{i}\right)
$$

where the function $f$ is called the activation function and is required to be differentiable. Sigmoid functions are commonly used as activation functions.

There are two classes of neural networks: feedforward and recurrent networks. In a feedforward neural network the neurons are connected together in a way in which information flows only in the forward direction, from inputs to outputs. The 


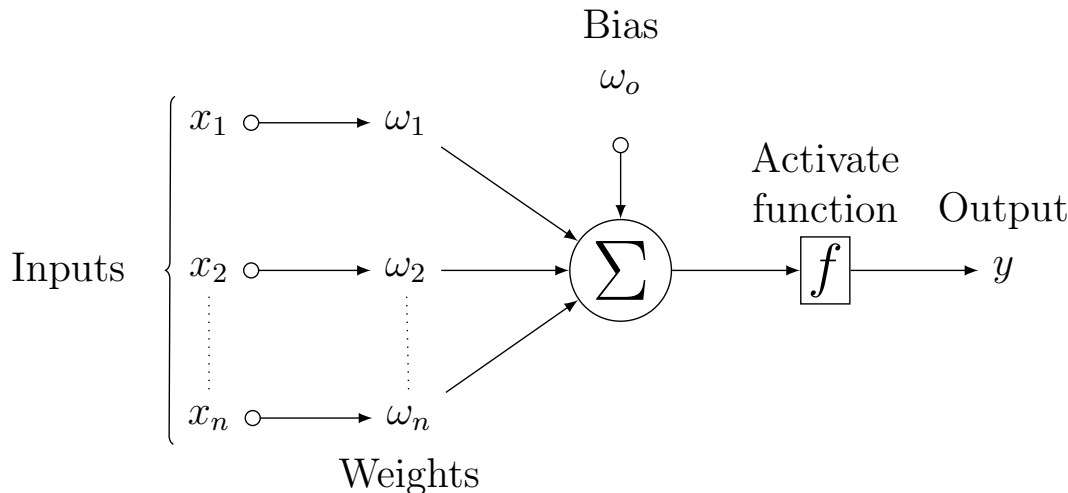

Figure 5.1: Diagram of a single neuron showing the inputs, weights and bias parameters taken by the function to generate an output.

neurons in the last layer are called the output neurons, and the other are the hidden neurons. Thus multiple neurons can take the inputs of the network and be connected to more layers of neurons to create a multilayer network. Recurrent or feedback neural networks on the other hand allow connections to go back in the flow, thus creating cycles. For this reason they are time dependent.

Neural networks can be used for applications where a nonlinear relation between numerical data needs to be found, the number of input variables is large (usually more than 3), an appropriate number of examples is available and no specific model exits.

\subsubsection{Training of Neural Networks}

Training is the process by which the parameters of the network are estimated. Different algorithms have been implemented which are basically optimization algorithms that minimize a cost function of the form:

$$
J(\omega)=\sum_{k=1}^{N}\left(y_{p}^{k}-g\left(x^{k}, \omega\right)\right)^{2}
$$


where $x^{k}$ is the vector of the values of the variables for example $k, y_{p}^{k}$ is the corresponding measured value of the quantity to be modeled, $\omega$ is the vector of the parameters (or weights) of the model, and $g\left(x^{k}, \omega\right)$ is the output value of the model with parameters $\omega$ for the vector of variables $x^{k}$. Therefore, the cost function is a function of all adjustable parameters $\omega$ of the model. Training consists in finding the parameter vector $\omega$ for which $J(\omega)$ is minimum.

The optimization techniques applied for neural networks are usually some type of gradient methods, with backpropagation being one of the most popular for computing the gradient of the cost function.

The training process then consists of the following steps:

1. Initialize all the parameters

2. Compute gradient of the cost function

3. Upgrade parameters iteratively with minimization algorithm

4. If maximum number of epoch is reached or weight no longer changes significantly or minimum has been reached, stop training.

\subsection{Methodology}

For the problem of finding a relation between the normal force distribution and the shear components of the GRF, the following procedure was followed to design the neural network:

1. Select the relevant inputs;

2. Collect the necessary data for training and testing;

3. Find the appropriate complexity of the model, i.e., the appropriate number of hidden neurons. This is very important, because with the increase of complexity 
we may fall in the overfitting problems, but if is not enough, no learning will occur. This is known as the bias-variance dilema [58]. One recommendation is that the number of parameters should be small compared with the number of elements in the training set;

4. Train the network; and

5. Assess the generalization of the network.

\subsubsection{Input selection}

For the estimation of the shear components of the ground reaction force we selected as input variables the individual forces measured by each sensor on the insole, thus resulting in an input vector of 14 variables, $F_{i}$.

Both the input forces from the sensors and the shear components of the GRF were normalized by body weight to be able to compare and use data between different subjects, but also to improve the performance of the training algorithms, since large values may saturate the sigmoid functions and slow down the training process.

\subsubsection{Data collection}

For the purpose of investigating the feasibility of estimating the shear forces from the insole measurements, data was collected from one subject for the training of the neural network. The subject wore the instrumented shoes with the insoles and walked along a walkway where 2 Bertec force plates were installed. Data was simultaneously recorded from the insole system and the force plates for 15 steps. The force measurements from the individual sensors were used as inputs of the network and the shear components measured by the force plate were used as targets in the training process. 


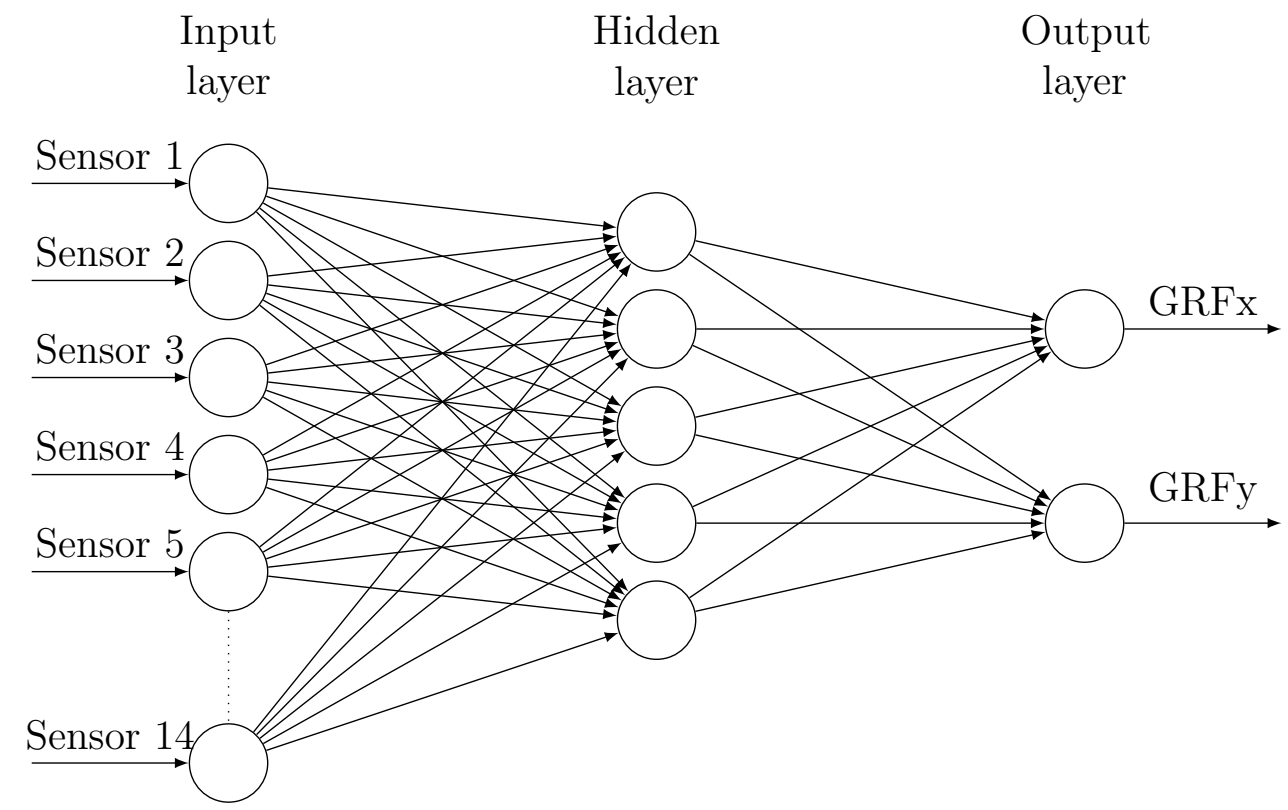

Figure 5.2: Neural Network implemented for estimation of shear components of GRF.

\subsubsection{Model selection}

A feedforward neural network such as the one shown on Figure 5.2 was chosen to find a nonlinear regression model for the estimation of the ground reaction forces.

We started with a single hidden layer with hyperbolic tangent sigmoid as the activation function for the neurons, and an output layer with linear function. The hyperbolic tangent function was chosen because is differentiable and the outputs were expected to vary from -1 to 1 , due to the bidirectional nature of the shear forces during walking.

The optimal number of hidden neuron was found experimentally starting with 10 neurons and then increasing or decreasing the number of neurons to evaluate different network sizes. The model with less neurons was chosen between networks with similar performance. This is called heuristically tuning the structure of the ANN. 


\subsubsection{Training and assessment of ANN}

The neural network toolbox from Matlab was used for the training of the network. The toolbox provides different options to create, customize and train a neural network. The number of layers, number of neurons and transfer functions can be chosen according to the desired model. Different training algorithms are available such as Levenberg-Marquardt, Bayesian Regularization and BFGS Quasi-Newton; and their parameters such as the learning rate can be customized.

It is important to verify that the ANN can work after it is fully trained, therefore, we split the data in 3 sets:

- Training (60\%). This set of data was used to train the network.

- Validation (20\%). This set was used to stop the training process when the error on the validation set stopped decreasing in order to avoid overfitting.

- Testing (20\%). This set of data was used to assess the neural network.

Training then was repeated with different testing sets, until all steps were used in the testing set. Four different sizes of networks were trained using 3, 5, 10 and 15 neurons on the hidden layer to compare the generalization using the LevenbergMarquardt algorithm. The correlation values, RMSE and, \%RMSE obtained were compared to select the appropriate number of neurons. Table 5.1 shows these results.

As can be seen in Table 5.1, the correlation values for the 5 neuron network were higher compared with the other networks sizes. In terms of the RMSE, again the 5 neuron network showed smaller errors in the A/P shear force and only the 15 neuron network had slightly smaller errors in the M/L shear force. Therefore, 5 neurons were selected for the hidden layer of the neural network. Thus, by selecting a network with just the enough number of neurons, the generalization of the model improves and we avoid overfitting the model [58]. 


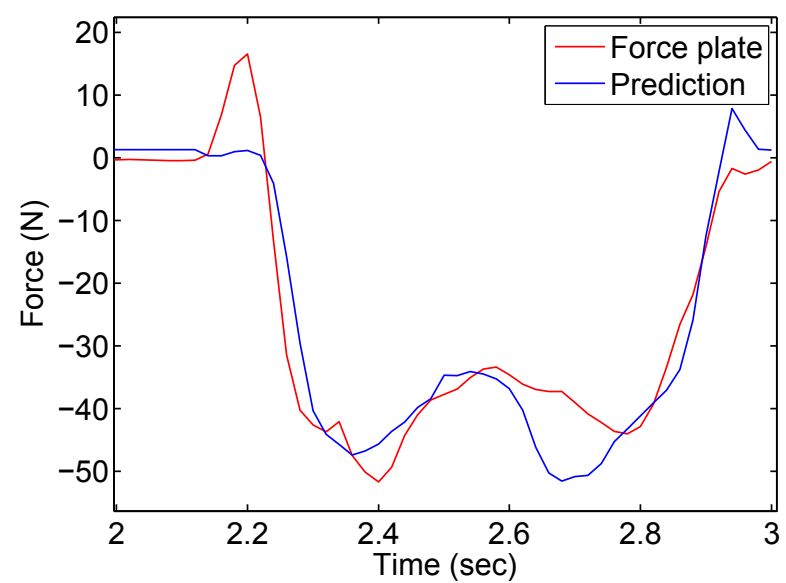

(a) Medial-Lateral GRF

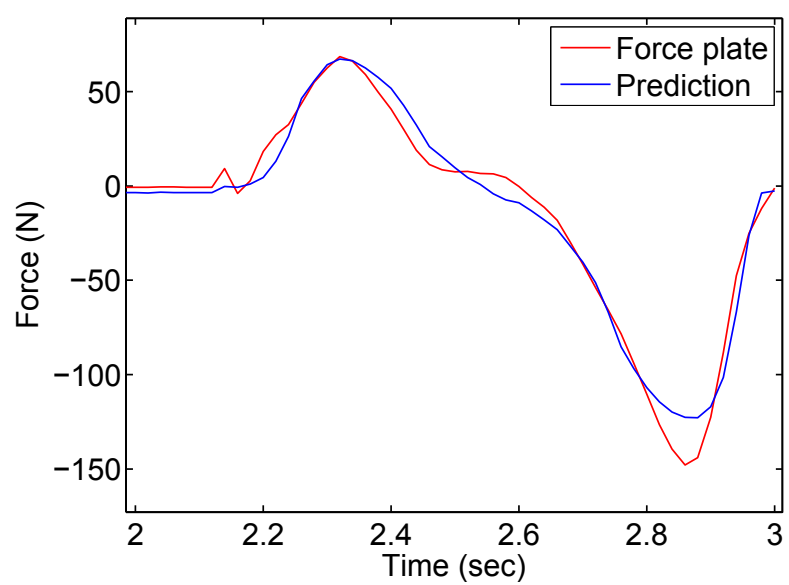

(b) Anterior-Posterior GRF

Figure 5.3: Estimated shear forces from the neural network compared with the force plate values.

An example of the results obtained for the medial/lateral and anterior/posterior shear forces from the neural network for one step of the testing set is shown on Figure 5.3. High correlation values (above 0.9) and errors around 6\% were obtained for the $\mathrm{A} / \mathrm{P}$ shear force, which shows that overall trend of the measurements was captured by the ANN. In the case of the M/L shear force, the results were good, with correlation values above 0.8 and errors around $10 \%$. These results showed that the neural network was able to estimate both shear components of the GRF from the

Table 5.1: Correlation and RMS errors of the estimated shear forces compared with the force plate measurements for different network sizes.

\begin{tabular}{|c|c|c|c|c|c|c|}
\hline & \multicolumn{3}{|c|}{ M/L shear force } & \multicolumn{3}{c|}{ A/P shear force } \\
\hline \# neurons & Correlation & RMSE (N) & \%RMSE & Correlation & RMSE (N) & \%RMSE \\
\hline 3 & 0.83 & 10.69 & 13.76 & 0.95 & 15.73 & 7.22 \\
\hline 5 & 0.87 & 9.90 & 12.66 & 0.96 & 12.26 & 5.64 \\
\hline 10 & 0.84 & 10.95 & 13.96 & 0.94 & 13.34 & 6.16 \\
\hline 15 & 0.84 & 9.55 & 12.14 & 0.95 & 13.06 & 6.01 \\
\hline
\end{tabular}


data from the individual force sensors; the correlation values and \%RMSE being better for the anterior/posterior component. This will also be confirmed in the upcoming experiments of Chapter 6 with more subjects.

The limitation of the current methodology is that the training was only performed using data from walking trials, thus, other motions may not be captured with the current approach.

\subsection{Summary}

This chapter presented a methodology for estimation of the shear forces that can be implemented in the force sensing insole to provide additional information that could be used for the assessment of balance. Good results were obtained for the M/L shear force and high correlation and low errors for the A/P shear force. These results showed the feasibility of using an ANN for calculation of the shear forces during walking. Verification experiments with different subjects are presented on the next chapter. 


\section{Chapter 6}

\section{Experiments with the force sensing insole}

The experiments in Chapter:4 and 5, were used as a preliminary evaluation of the insole and to make the improvements on the design. The objective of the experiments presented in this chapter was to conduct a more comprehensive measurement of the GRF and the COP of the insole on both standing and walking conditions with healthy subjects.

This study was approved by the Carleton University Research Ethics Board (file number 101290, see Appendix A and all subjects provided informed consent. The experiments were carried out in the Advanced Biomechatronic and Locomotion Lab at Carleton University.

Two Bertec force plates placed along a walkway, shown in Figure 6.1, were used to measure the ground reaction forces, and the Optotrak Certus Motion Capture System was used to track the position of the feet over the force plates for comparison with the insole measurements.

Seven young female healthy participants with US shoe sizes between 6 to 9 were invited to participate in this study. This group was selected for practical purposes to be able to test the particular size of the insole prototype. The weights and shoe sizes of the participants are shown in Table 6.1. 


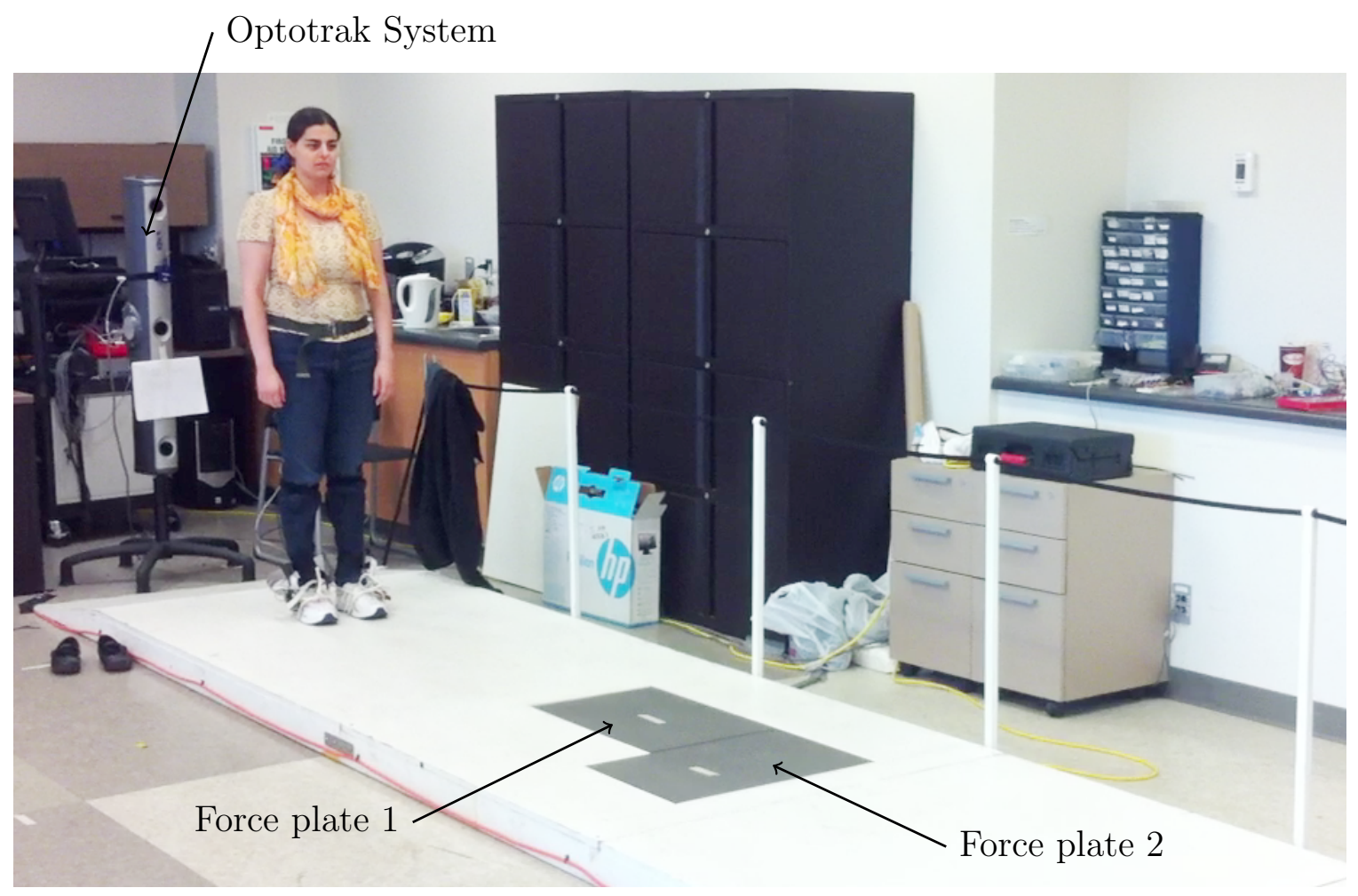

Figure 6.1: Walkway with the two Bertec force plates.

Table 6.1: Characteristics of participants.

\begin{tabular}{|l|c|c|}
\hline & Weight (kgf) & US Shoe size \\
\hline Subject 1 & 61.8 & 8 \\
\hline Subject 2 & 83.9 & 7 \\
\hline Subject 3 & 56.3 & 6.5 \\
\hline Subject 4 & 80.1 & 9 \\
\hline Subject 5 & 56.2 & 7 \\
\hline Subject 6 & 56.7 & 6.5 \\
\hline Subject 7 & 67.9 & 6.5 \\
\hline
\end{tabular}


For all the experiments, participants were asked to wear a pair of shoes containing the force sensing insole, an outer structure with infrared markers and a small camera for the localization system [40], the Raspberry Pi with the ADC, and an Optotrak marker. Additionally, a small box containing the electronics for the force sensors was attached to the ankle of the participants by means of a velcro strap. Finally, the participants were asked to carry a belt containing the battery to power the system and the wireless transmitter for the Optotrak system. A user wearing the set up is shown in Figure 6.2.

\subsection{Standing experiments}

For the standing experiments, participants were instructed to stand over one force plate as still as possible on two feet, one foot and on toes. GRF and COP were collected for about 15 seconds from both systems for two different conditions: eyes opened and eyes closed. Data was simultaneously collected from the force sensing insoles, the force plate and the Optotrak System. The objective of this experiment was to analyze the ability of the force sensing insole to measure the variation of the body sway in different situations and base of supports compared to the measurements from the force plate for eventual assessment of balance.

Data from the insole was collected at $50 \mathrm{~Hz}$. Data from both the force plate and the Optotrack system were collected at $100 \mathrm{~Hz}$. The force plate data was then resampled at $50 \mathrm{~Hz}$ by interpolation for comparison with the insole measurements. Subjects were instructed to stand outside the force plate at the beginning of each test condition and then make a step on the force plate in order to synchronize the data from both systems. For this experiment, data from both the force plate and the insole was filtered using a second-order Butterworth filter with cutoff frequency of 5 


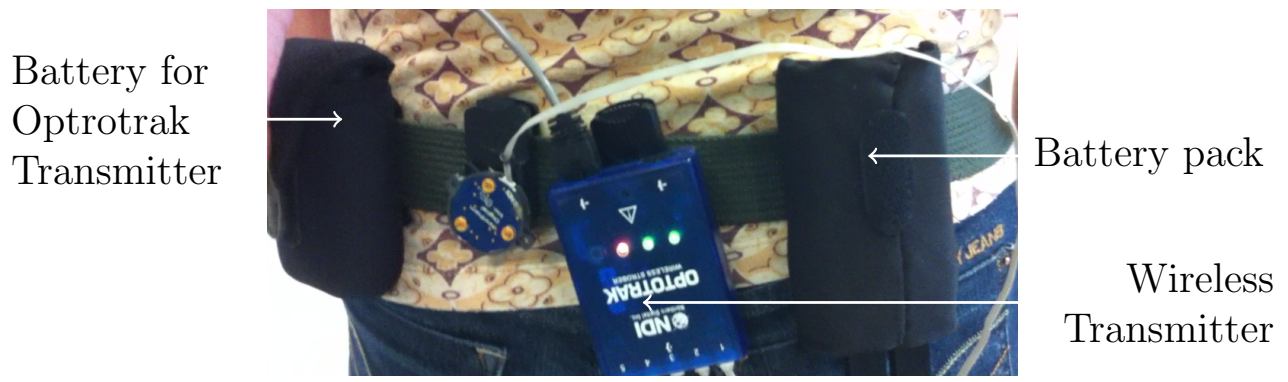

(a)

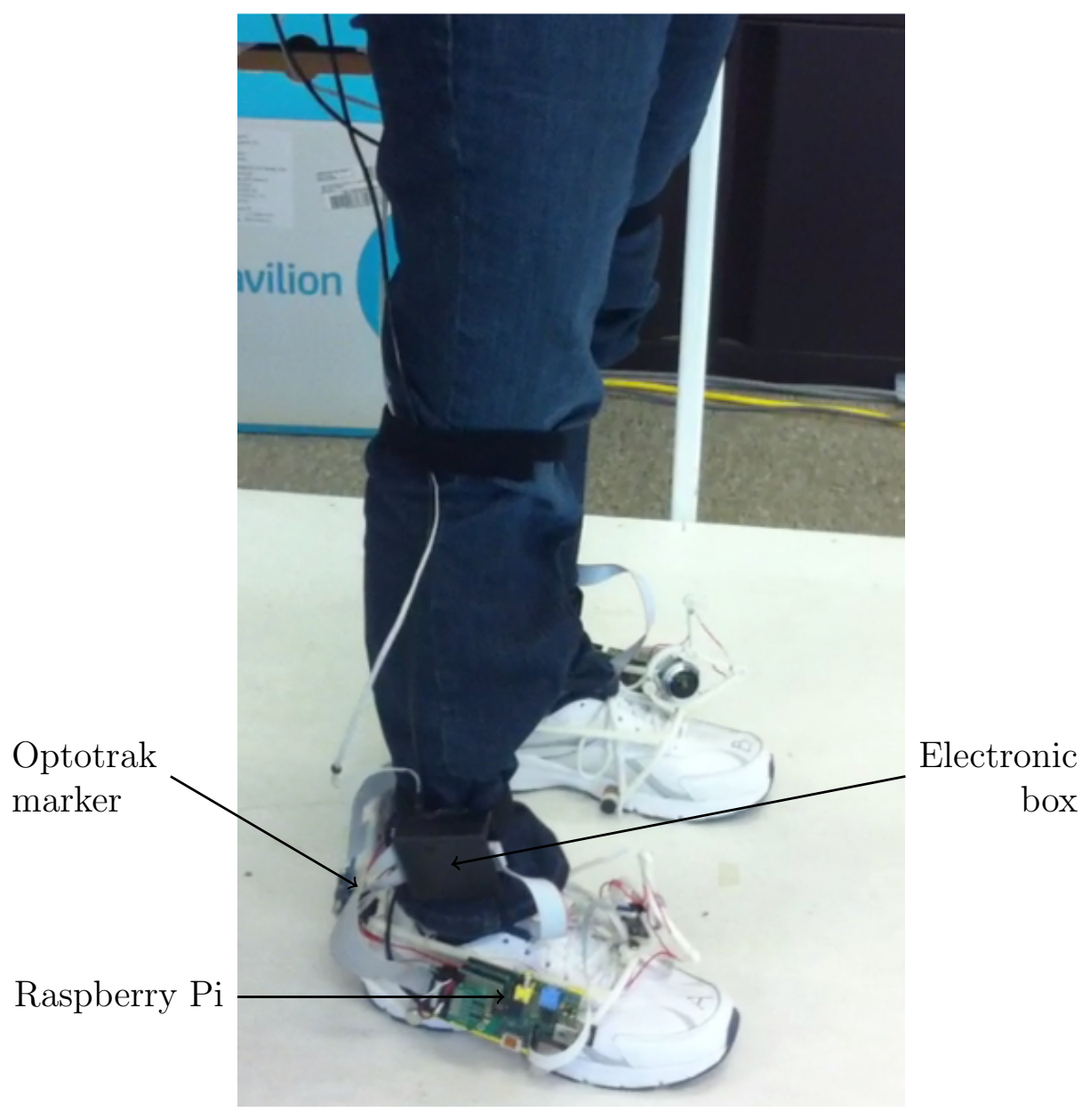

(b)

Figure 6.2: Set up used by participants: (a) Belt with the battery to power the system and wireless transmitter for the Optotrak. (b) Instrumented shoe with Raspberry Pi and electronic box attached to the ankle. 
Hz. Throughout the experiments, the position of the shoes over the force plate were tracked using the Optotrak markers on the shoes to transform the measurement from the insole frame to the force plate frame as described in Section 6.2.1 and to calculate the net center of pressure as:

$$
C O P_{n e t}=C O P_{l} \frac{R_{v l}}{R_{v l}+R_{v r}}+C O P_{r} \frac{R_{v r}}{R_{v l}+R_{v r}}
$$

where $C O P_{l}$ and $C O P_{r}$ are the center of pressure under the left and right feet respectively. $R_{v l}$ and $R_{v r}$ are the vertical ground reaction forces under the left and right foot respectively.

\subsubsection{Results}

As a measure of postural steadiness, the standard deviation of the time series for both the anterior/posterior $(\mathrm{A} / \mathrm{P})$ and medial/lateral $(\mathrm{M} / \mathrm{L})$ direction of the COP was calculated to quantify the COP sway. Table 6.2 shows the median and interquartile measurements from both the insole and the force plate for the 7 subjects. It can be appreciated that as the standing conditions becomes more challenging (eyes closed or decrease on base of support area by rising to toes or standing on one foot) the variation on the position of the COP increases. These changes were observed on both the insole and force plate recordings as can be seen in Figure 6.3 for one example subject in the one foot standing condition. Similar trends for the rest of the subjects can be found in Appendix $\mathrm{D}$.

Although the range of variation on the insole is somehow smaller than the force plate measurements, it is still able to differentiate the six different standing conditions, thus providing information that could be used for balance assessment. Figure 6.4 
Table 6.2: Median and interquartile values of the variation of COP position in the $\mathrm{A} / \mathrm{P}$ and $\mathrm{M} / \mathrm{L}$ direction for the 3 different foot configuration with eyes opened (EO) and eyes closed (EC) for the seven participants.

\begin{tabular}{|l|c|c|c|c|}
\hline & \multicolumn{2}{|c|}{ Insole } & \multicolumn{2}{c|}{ Forceplate } \\
\hline & COPy $(\mathrm{mm})$ & COPx $(\mathrm{mm})$ & COPy $(\mathrm{mm})$ & COPx $(\mathrm{mm})$ \\
\hline 2 feet EO & $1.39(1.07)$ & $0.96(0.36)$ & $3.81(1.38)$ & $2.94(1.32)$ \\
\hline 2 feet EC & $1.10(3.70)$ & $6.06(0.73)$ & $2.91(2.83)$ & $7.30(1.10)$ \\
\hline 2 toes EO & $7.30(1.45)$ & $6.71(3.13)$ & $10.46(3.89)$ & $6.84(0.89)$ \\
\hline 2 toes EC & $11.56(5.54)$ & $13.48(10.28)$ & $18.12(9.49)$ & $10.88(5.23)$ \\
\hline 1 foot EO & $5.70(1.57)$ & $4.31(1.28)$ & $7.67(0.53)$ & $6.16(2.24)$ \\
\hline 1 foot EC & $11.25(6.55)$ & $6.45(1.42)$ & $13.77(4.53)$ & $10.11(1.25)$ \\
\hline
\end{tabular}

shows an example of the $C O P_{n e t}$ from both systems in the 6 different conditions from one subject.

\subsubsection{Discussion}

From Table 6.2 it can be observed that the most unstable or challenging standing position was on toes with the eyes closed, where the COP showed the greatest range of sway. In this case, the difference on sway between subjects increased (notice the higher value on standard deviation for this test condition), and while for some subjects the range of sway was greater on the $\mathrm{A} / \mathrm{P}$ direction, for others the inverse was the case, this explains the difference on trend for this standing condition. For all foot configurations and for all subjects, the condition for eyes closed showed a greater sway on the COP compared to with eyes opened. Although the ranges of COP sway recorded by the insole were slightly smaller than the those measured by the force plate, the measurements show a good correspondence and the insole is able to track the changes on the $\mathrm{COP}$ in both directions as shown in Figure 6.3. 


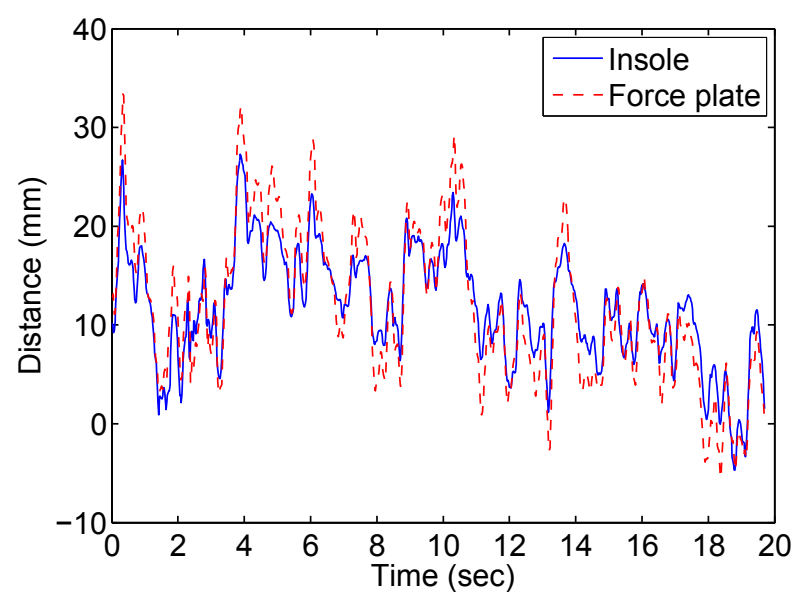

(a) $\mathrm{COP}$ in the $\mathrm{A} / \mathrm{P}$ direction

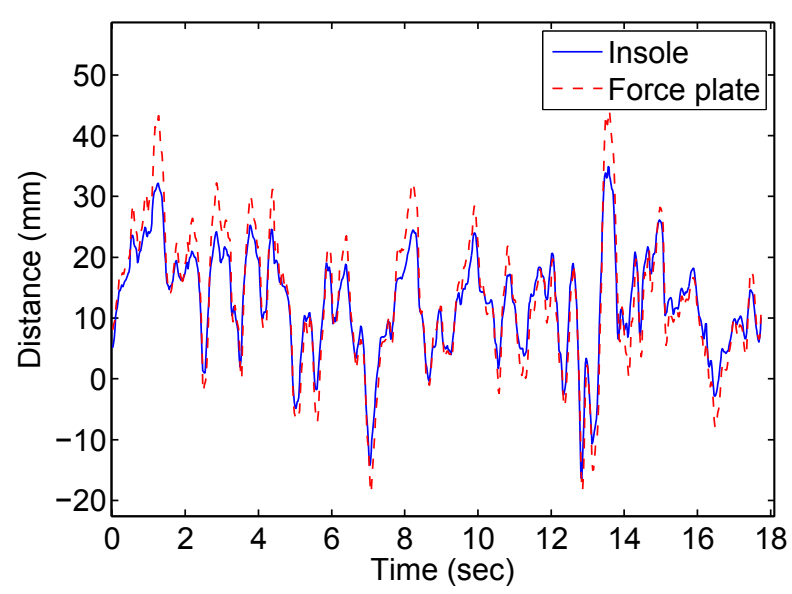

(c) $\mathrm{COP}$ in the $\mathrm{A} / \mathrm{P}$ direction

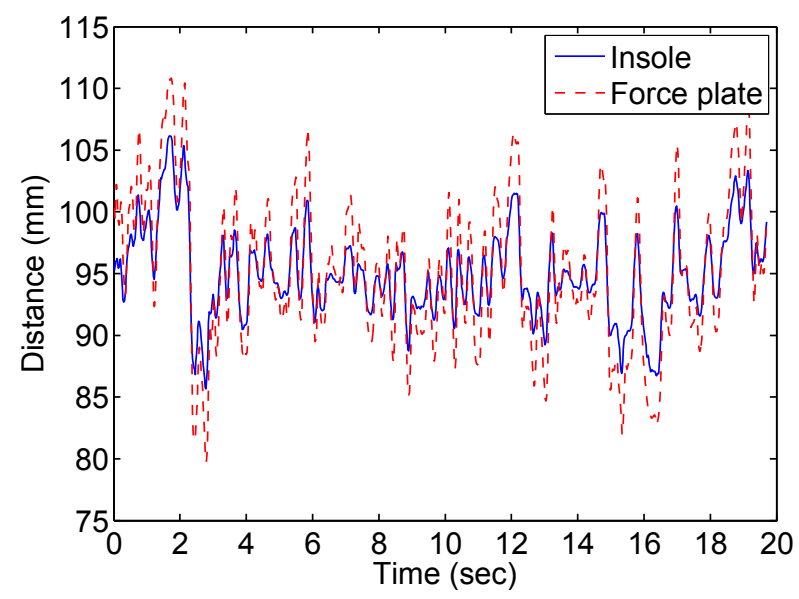

(b) $\mathrm{COP}$ in the $\mathrm{M} / \mathrm{L}$ direction

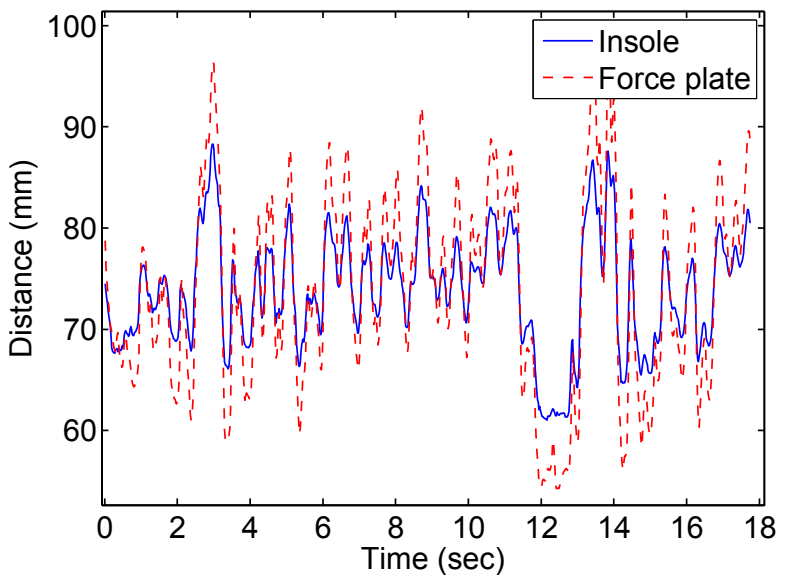

(d) $\mathrm{COP}$ in the $\mathrm{M} / \mathrm{L}$ direction

Figure 6.3: Comparison of insole (blue stars) and force plate (red stars) measurements of COP from subject 1 standing on one foot with eyes opened (a), (b) and with eyes closed (c), (d). The blue circles represent the position of the sensors on the insole. 


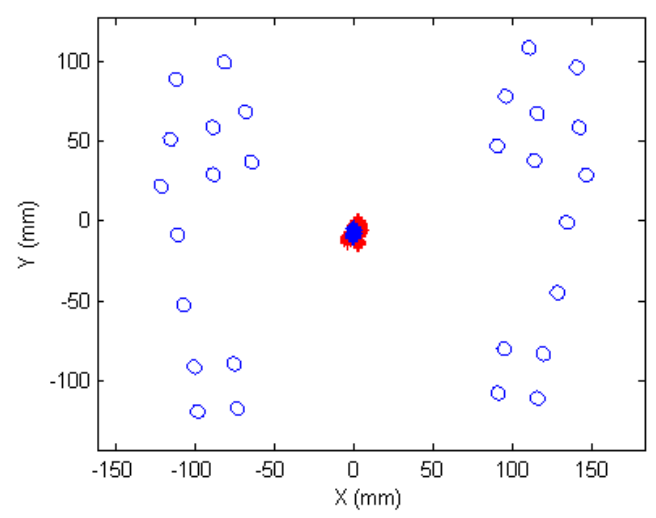

(a) 2 feet eyes opened

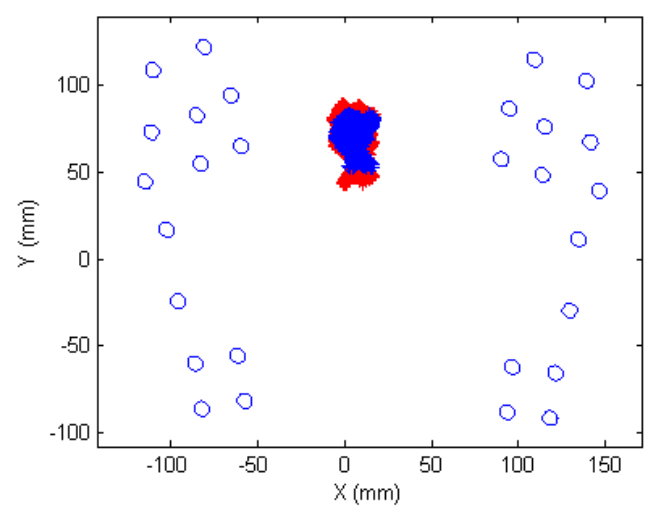

(c) 2 toes eyes opened

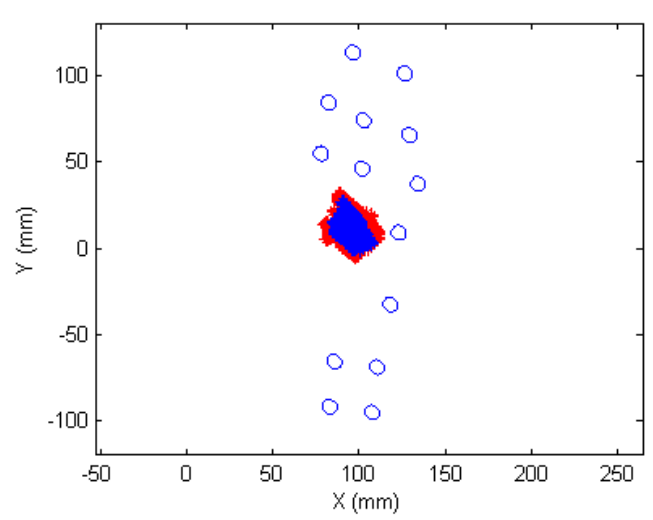

(e) 1 foot eyes opened

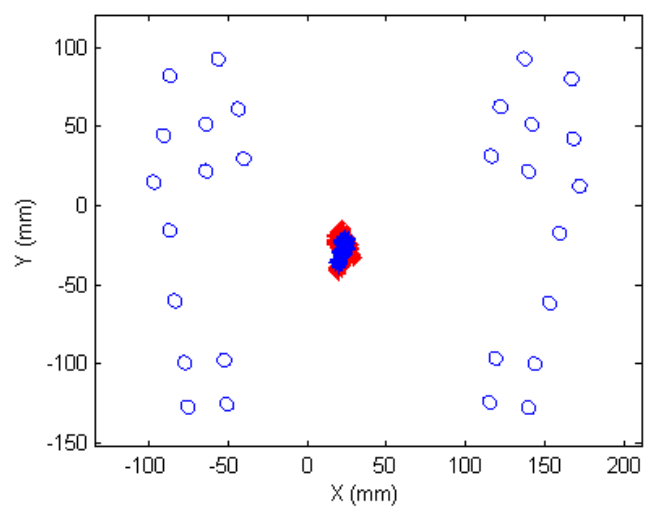

(b) 2 feet eyes closed

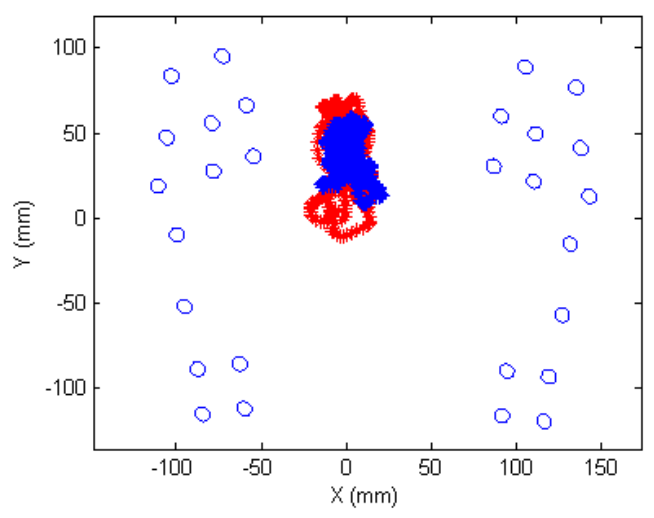

(d) 2 toes eyes closed

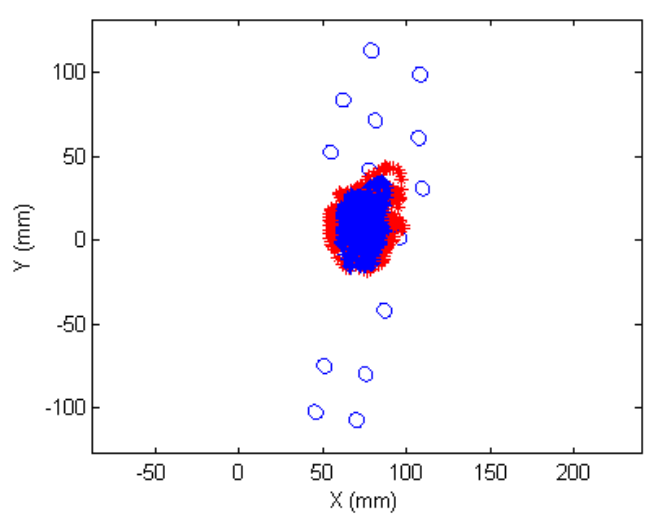

(f) 1 foot eyes closed

Figure 6.4: Comparison of insole (blue) and force plate (red) measurements of COP from subject 1 during standing experiments. 


\subsection{Walking experiments}

For this experiment, participants were asked to walk along the walkway where 2 Bertec force plates were installed while wearing the instrumented shoes. Simultaneous data was recorded from both insoles and force plates, as well as the Optotrak markers. Ten walking trials were performed per participant at their preferred speed. The objective of this experiment was to validate the measurements of the insole with healthy subjects (both the GRF and COP) during walking. The data collected was also used for estimation of the shear components of the ground reaction force with the application of neural networks as presented in Chapter 5.

The data collected was divided into steps, from heel strike to toe off by applying a threshold on the total vertical ground reaction force (VGRF). The COP was calculated as presented in Section 4.2 and the values outside the insole were disregarded to compensate for the difference in length between the shoe and the insole.

During the analysis of the data it was observed that the output of the sensors varied over time, affecting the overall scale of the measurements. This could be due to the excessive use of the sensors, rubber yielding, or various deformations. These errors were overcome by means of a pre-test calibration. The first five steps were then used to fit a model for the calculation of the VGRF, so that each sensor was scaled accordingly for each experiment session such that:

$$
V G R F=\sum_{i=1}^{n} c_{i} F_{i}
$$

where $F_{i}$ is the force in the $i$ sensor calculated with the calibration values obtained in Section 4.1 and $c_{i}$ is the additional coefficient weight. 

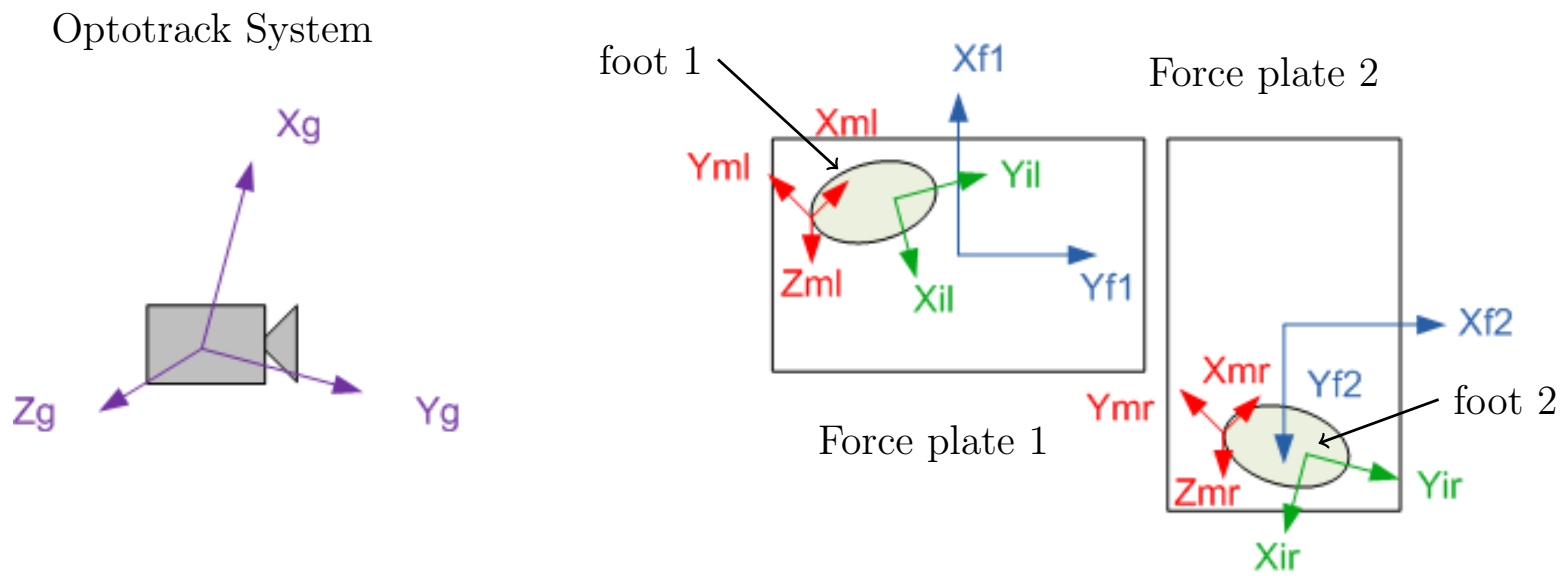

Figure 6.5: Top view of the Optotrak, force plate and shoes with their coordinate systems.

\subsubsection{Coordinate System transformations}

Because the foot lands in different orientations on each walking trial and also to be able to compare data from different subjects, the shear components of the ground reaction force and $\mathrm{COP}$ measured by the force plate must be aligned with the coordinate system in the insole. In order to do this, the Optotrak marker attached to the instrumented shoe was used to calculate the position and orientation of the foot when landing. Figure 6.5 shows a diagram of the coordinates system of the Optotrak $\left(X_{g}, Y_{g}, Z_{g}\right)$, force plates $\left(X_{f}, Y_{f}, Z_{f}\right)$, insoles $\left(X_{i}, Y_{i}, Z_{i}\right)$ and markers $\left(X_{m}, Y_{m}, Z_{m}\right)$. In this case we needed to find the transformation matrices required to convert the measurements from the coordinate system of the force plate to the insole frame.

The position of the force plate was previously recorded with the Optotrak system to obtain the transformation matrix with respect to the global coordinate system of the Optrotrak so that:

$$
P_{g}=v_{g}+q_{g f} P_{f} q_{f g}
$$

where $P_{f}$ is a point on the force plate coordinate system, $v_{g}$ is the translation vector from the origin of the global coordinate system of the Optotrack to the origin of the 
coordinate system of the force plate (measured once) as shown in Figure 6.6. $q_{g f}$ is the quaternion for the rotation matrix from the global coordinate system to the force plate and $q_{f g}$ is the inverse quaternion.

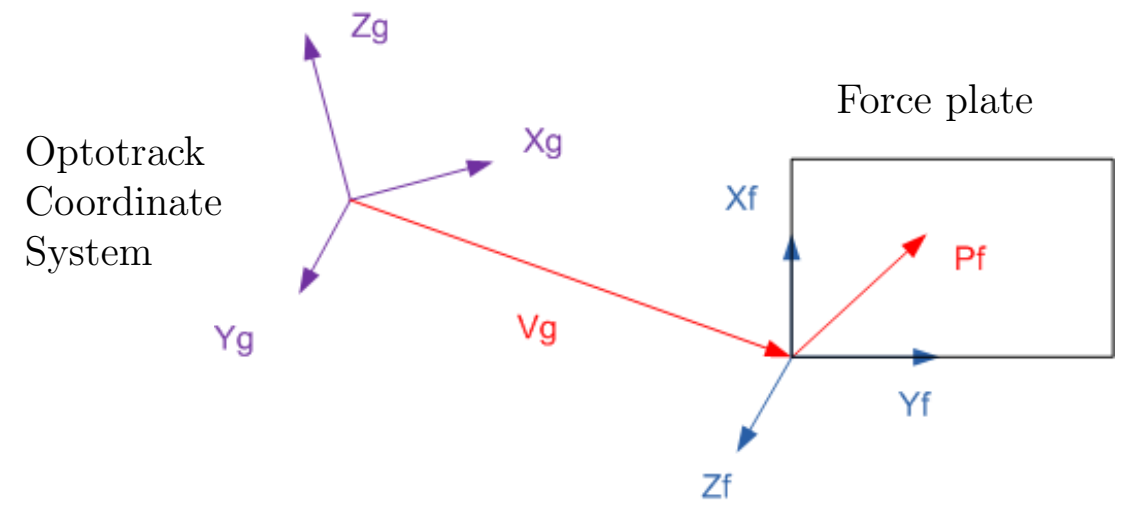

Figure 6.6: Transformation from global coordinate system of the Optotrak and one of the force plates.

Similarly, the position of the insole was measured with respect to the marker on the shoe to obtain the corresponding transformation as shown in Figure 6.7 so that:

$$
P_{i}=v_{i}+q_{i m} P_{m} q_{m i}
$$

where $q_{i m}$ is the quaternion for the rotation matrix from the insole frame to the marker.

Marker Coordinate System

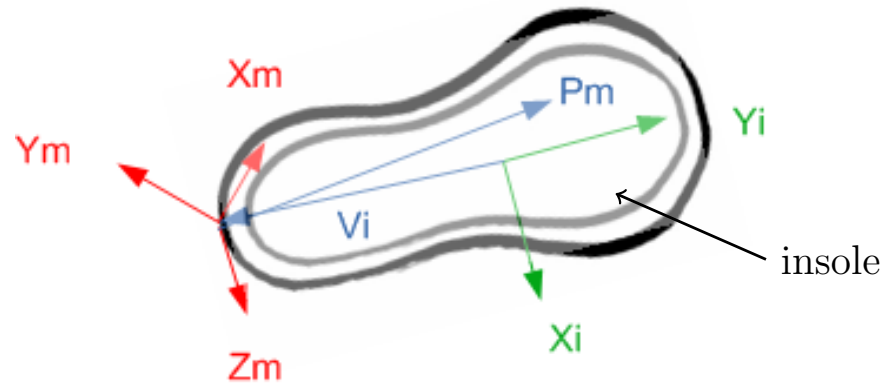

Figure 6.7: Transformation from marker coordinate system to one of the insoles. 
To calculate these two transformation matrices the Davenport's method [59] was used as described in Appendix C. This method requires to know three points on each coordinate system in order to obtain the transformation matrix between them.

Finally, having both the transformation matrices from the force plates to the global coordinate system of the Optotrak, and from the markers on the shoe to the insole, we were able to track the position of the shoes on the force plate with the use of the Optotrak markers. In the case of the transformation from the global coordinate system of the Optotrack to the marker, both the translation vector and the orientation matrix was provided by the software, so that:

$$
P_{m}=v_{m}+q_{m g} P_{g} q_{g m}
$$

where $q_{m g}$ is the quaternion for the rotation matrix from the marker to the global coordinate system and $v_{m}$ is the translation vector. Thus, by combining this coordinate systems transformations, the position of measurements from the force plate can be transformed from their coordinate system to the insole frame.

\subsubsection{Shear forces estimation}

For the estimation of the shear components of the GRF, an artificial neural network was trained as explained in Chapter 5. The medial-lateral component of the GRF from the right foot was flipped to have comparable data from both sides. Two training strategies were implemented:

1. An intra-subject strategy. Data from the same participant was divided into three sets and a three fold cross validation was implemented, so that for each fold two sets were used as the training data, and the third one was used as the testing data. This was repeated until all three sets were used as the testing set. 
2. An inter-subject strategy. Data from the others participants was used for training. The total set of data consisted of 10 steps per side per subject.

\subsubsection{Results}

The RMS errors and correlation coefficients were calculated for the VGRF and COP in the $\mathrm{A} / \mathrm{P}$ and $\mathrm{M} / \mathrm{L}$ direction. Tables 6.3 and 6.4 show the mean and standard deviation for each subject for a total of 10 steps ( 5 for each side), with high correlation values (above 0.9) indicating a good match between the trend of the signals.

Figure 6.8 shows an example of the VGRF, the COP on the insole frame and the $\mathrm{COP}$ in the $\mathrm{A} / \mathrm{P}$ and $\mathrm{M} / \mathrm{L}$ direction over time for one step from one subject. Similar

plots for the rest of the subjects can be found in Appendix $\mathrm{D}$.

On the other hand, The RMS errors and correlation coefficients were calculated for the two shear components of the GRF estimated by the neural network. Tables 6.5 and 6.6 show the mean and standard deviation for each subject for the 10 trials for the intra-subject and inter-subject training strategies respectively.

Finally, Figure 6.9 shows one example of the the A/P and M/L shear forces estimation from the ANN for one step for one subject for the intra-subject strategy.

\subsubsection{Discussion}

As shown in Table 6.3, the results from the VGRF showed a good correspondence with the force plate, with a mean error of $6.77 \%$ of the total force measured by each subject and mean correlation value of 0.96. For the COP shown in Table 6.4, the correlations in the $\mathrm{M} / \mathrm{L}$ direction were fair, with a mean of 0.69 , but a mean RMSE 
Table 6.3: Correlation and RMS errors of VGRF for the participants.

\begin{tabular}{|l|c|c|c|}
\hline & \multicolumn{3}{|c|}{ VGRF } \\
\hline & Corr & RMSE (N) & \%MSE \\
\hline Subject 1 & $0.9902(0.0055)$ & $30.3324(6.4592)$ & $4.8912(1.0399)$ \\
\hline Subject 2 & $0.8677(0.3387)$ & $36.3929(17.7552)$ & $4.3615(2.1218)$ \\
\hline Subject 3 & $0.9533(0.0338)$ & $63.1609(26.1657)$ & $11.4065(4.8916)$ \\
\hline Subject 4 & $0.9901(0.0033)$ & $44.2907(12.3333)$ & $5.0541(1.4105)$ \\
\hline Subject 5 & $0.9819(0.0089)$ & $47.4489(15.6005)$ & $7.8184(2.6146)$ \\
\hline Subject 6 & $0.9912(0.0053)$ & $29.0258(7.9404)$ & $5.0073(1.3031)$ \\
\hline Subject 7 & $0.9714(0.0120)$ & $64.3374(17.4778)$ & $8.8626(2.2663)$ \\
\hline
\end{tabular}

Table 6.4: Correlation and RMS errors of COP for the participants.

\begin{tabular}{|l|c|c|c|c|}
\hline & \multicolumn{2}{|c|}{ COPx } & \multicolumn{2}{c|}{ COPy } \\
\hline & Corr & RMSE $(\mathrm{mm})$ & Corr & RMSE $(\mathrm{mm})$ \\
\hline Subject 1 & $0.8663(0.0567)$ & $4.6507(1.3306)$ & $0.9336(0.0308)$ & $19.9567(4.1872)$ \\
\hline Subject 2 & $0.6663(0.2755)$ & $4.6149(1.8749)$ & $0.8146(0.3220)$ & $17.8219(8.1476)$ \\
\hline Subject 3 & $0.7644(0.0804)$ & $7.2942(1.8581)$ & $0.9470(0.0216)$ & $21.9869(4.1392)$ \\
\hline Subject 4 & $0.6883(0.1644)$ & $6.4622(1.2609)$ & $0.8888(0.0892)$ & $28.6518(9.0583)$ \\
\hline Subject 5 & $0.5958(0.2935)$ & $7.1406(1.7004)$ & $0.9322(0.2320)$ & $22.4928(4.2603)$ \\
\hline Subject 6 & $0.7343(0.1864)$ & $4.4075(1.1050)$ & $0.9649(0.0160)$ & $16.0300(2.8782)$ \\
\hline Subject 7 & $0.7227(0.0720)$ & $10.2761(1.8968)$ & $0.8835(0.1337)$ & $26.5042(12.2930)$ \\
\hline
\end{tabular}




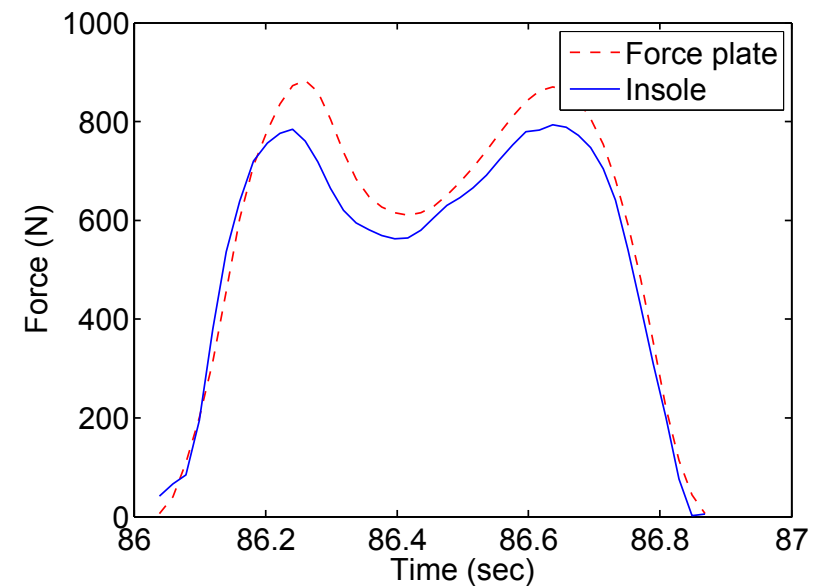

(a) VGRF

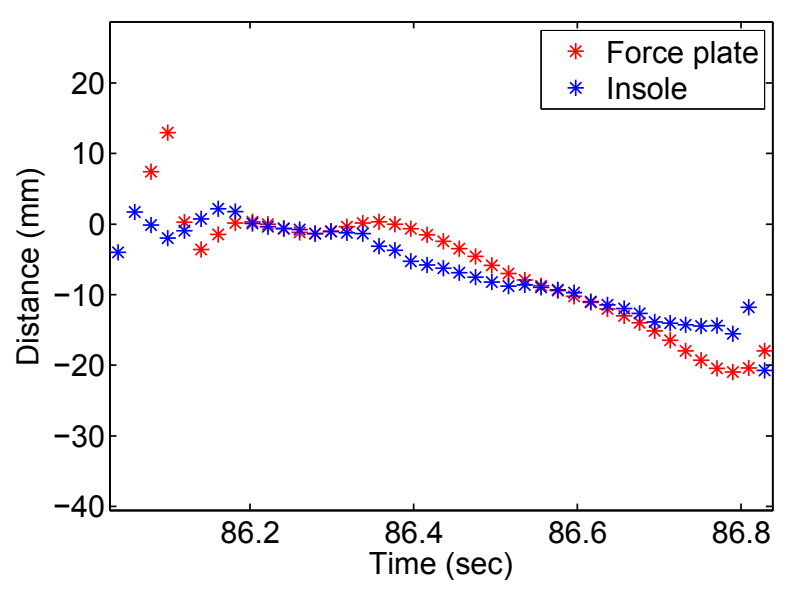

(c) $\mathrm{COPx}$

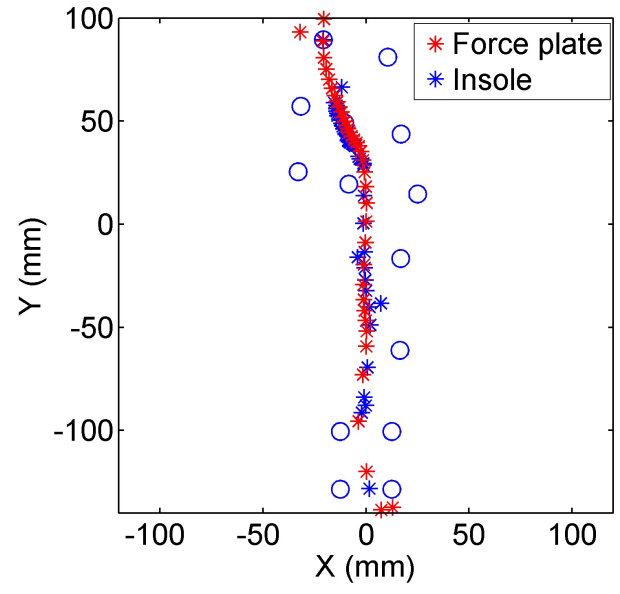

(b) COP

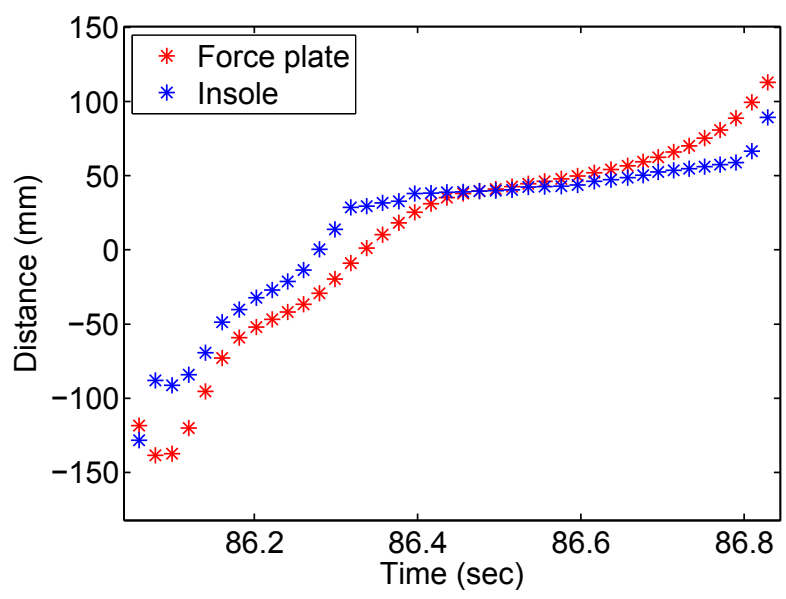

(d) $\mathrm{COPy}$

Figure 6.8: Comparison of insole (blue) and force plate (red) measurements of GRF and COP for one example step of the walking experiments from subject 4 . 
Table 6.5: Correlation and RMS errors of shear forces for the participants for intrasubject training strategy.

\begin{tabular}{|l|c|c|c|c|c|c|}
\hline & \multicolumn{3}{|c|}{ A/P force } & \multicolumn{3}{c|}{ M/L force } \\
\hline & Corr & RMSE (N) & \% RMSE & Corr & RMSE (N) & \% RMSE \\
\hline Subject 1 & $0.98(0.02)$ & $5.53(1.49)$ & $7.37(2.58)$ & $0.84(0.13)$ & $11.96(4.42)$ & $9.98(2.87)$ \\
\hline Subject 2 & $0.93(0.05)$ & $22.59(8.05)$ & $13.85(5.16$ & $0.87(0.07)$ & $9.81(2.77)$ & $12.92(2.89)$ \\
\hline Subject 3 & $0.86(0.10)$ & $23.44(6.95)$ & $16.92(6.34)$ & $0.63(0.21)$ & $10.59(4.09)$ & $20.62(10.01)$ \\
\hline Subject 4 & $0.97(0.03)$ & $27.16(16.77)$ & $9.15(5.70)$ & $0.86(0.06)$ & $10.86(3.75)$ & $12.17(4.74)$ \\
\hline Subject 5 & $0.94(0.05)$ & $17.11(4.35)$ & $12.62(3.71)$ & $0.81(0.13)$ & $7.66(2.30)$ & $16.27(5.69)$ \\
\hline Subject 6 & $0.95(0.05)$ & $15.81(7.71)$ & $10.23(5.07)$ & $0.87(0.09)$ & $8.00(2.62)$ & $14.39(6.06)$ \\
\hline Subject 7 & $0.89(0.09)$ & $28.99(10.02)$ & $13.74(4.74)$ & $0.83(0.15)$ & $14.93(4.65)$ & $15.91(6.38)$ \\
\hline
\end{tabular}

Table 6.6: Correlation and RMS errors of shear forces for the participants for intersubjects training strategy.

\begin{tabular}{|l|c|c|c|c|c|c|}
\hline & \multicolumn{3}{|c|}{ A/P force } & \multicolumn{3}{c|}{ M/L force } \\
\hline & Corr & RMSE (N) & \% RMSE & Corr & RMSE (N) & \% RMSE \\
\hline Subject 1 & $0.93(0.03)$ & $22.82(3.14)$ & $14.09(1.58)$ & $0.77(0.13)$ & $12.00(1.74)$ & $21.78(4.40)$ \\
\hline Subject 2 & $0.83(0.16)$ & $32.21(7.14)$ & $22.67(6.96)$ & $0.76(0.08)$ & $18.31(6.18)$ & $24.05(6.21)$ \\
\hline Subject 3 & $0.88(0.12)$ & $32.90(13.20)$ & $23.29(9.88)$ & $0.62(0.14)$ & $10.76(4.15)$ & $21.11(10.65)$ \\
\hline Subject 4 & $0.85(0.07)$ & $57.99(8.89)$ & $19.77(7.24)$ & $0.77(0.14)$ & $17.78(6.61)$ & $19.23(1.55)$ \\
\hline Subject 5 & $0.82(0.18)$ & $29.59(15.83)$ & $22.05(7.21)$ & $0.70(0.16)$ & $13.14(4.94)$ & $29.59(15.83)$ \\
\hline Subject 6 & $0.92(0.07)$ & $26.51(10.51)$ & $17.12(7.00)$ & $0.61(0.19)$ & $13.78(3.71)$ & $23.66(4.44)$ \\
\hline Subject 7 & $0.92(0.06)$ & $40.29(14.82)$ & $18.98(7.12)$ & $0.59(0.30)$ & $23.49(3.61)$ & $24.55(3.34)$ \\
\hline
\end{tabular}




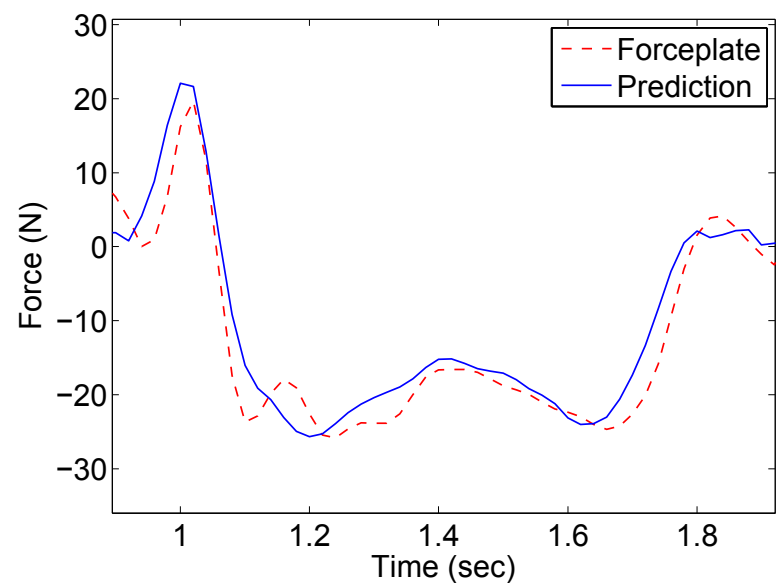

(a) Medial-Lateral GRF

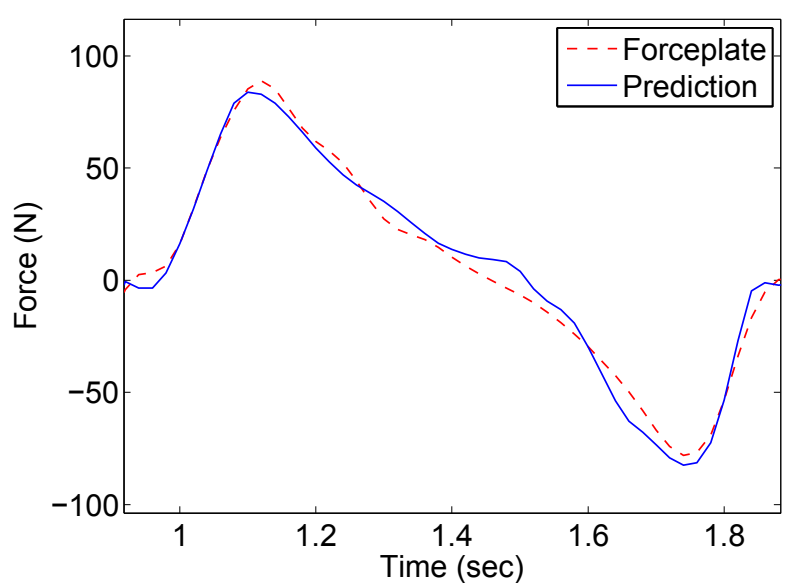

(b) Anterior-Posterior GRF

Figure 6.9: Estimated shear forces from the intra-subject training strategy of the neural network compared with the force plate values for subject 1.

of only $6.41 \mathrm{~mm}$. The correlation on the $\mathrm{A} / \mathrm{P}$ direction was good with mean value of 0.91 and mean RMSE of $21.92 \mathrm{~mm}$. An important factor that should be taken into account is that our sensor does not exactly measure what the force plate sees. One of the factors that contributed to the errors on the COP was that the range of COP measure on the insole is limited to the position of the sensors. Furthermore, it has been documented on the literature that the COP path differs slightly when the subjects step with shoes compared to barefoot condition on the force plate. Since the insole is placed inside the shoe and is in contact with the foot, and the force plate measures the COP of the participants wearing the shoes, there is somehow expected error between the measurements.

In the case of estimation of the shear components of the GRF, better results were obtained with the intra-subject training strategy than the inter-subject strategy, with mean correlation values of 0.93 and \%RMSE of $11.98 \%$ in the A/P direction and mean correlation values of 0.81 and \%RMSE of $14.61 \%$ in the M/L direction Table 6.5 . 


\subsection{Summary}

This chapter presented the experiments performed with the force sensing insole with seven healthy subjects. The results from the standing and walking experiments showed the capability of the insole to measure the changes and variations on the COP on different stability conditions as well as during locomotion. Furthermore, the insole was able to measure the VRGF within $7 \%$ error using the pre-test subject calibration and the method presented for estimating the shear components of the GRF during walking was shown to yield mean errors less than $15 \%$ using data from the same subject. The intra-subject training strategy can be used when calibration can be performed for the specific person before use of the device. However, if only a fair estimation is required, then the inter-subject strategy can be implemented by having a database with a larger number of subjects. The presented measurement strategy then seems suitable for application in the overall BalanceAid system. 


\section{Chapter 7}

\section{Conclusions and Future Work}

The BalanceAid project was conceived with the aim of developing a balance enhancement system capable of assessing the stability of the user and preventing a fall. Required measurements for this project are the ground reaction force and center of pressure. These measurements can be used for stability analysis and to provide sensory information to the user by means of vibrotactile feedback.

Mobile solutions for measuring the ground reaction forces for gait analysis are emerging, so that continuous steps can be collected and analyzed outside the laboratory environment. However, there are still limitations on the current systems, since some of the devices only obtain a partial measurement of the vertical ground reaction force, or in other cases, due to the thickness and rigidity of the device, they cause changes on the normal gait patterns.

This thesis presented the design and evaluation of a force sensing insole that addresses some of these limitations in order to obtain a measurement of the complete ground reaction forces and COP for use on a balance enhancement system.

\subsection{Conclusions}

On overview of the conclusions drawn from this research work is presented below. 


\section{Design and Prototyping of the sensor}

For the design of the force sensing insole, both a simulation of the forces under the foot from Section 3.3.5 and the experiments with the first prototype described in Section 4.2 provided valuable information that was used to decide the final number of sensors and the thickness of the insole. The resulting insole was built using molded rubber and 14 thin force sensors. The insole is able to concentrate the forces on the contact areas of the sensors, while remaining thin and flexible to allow normal walking. Furthermore, the implementation of the real-time data acquisition system allows the integration of the sensor with other components of the BalanceAid project.

\section{Calibration and Preliminary testing}

Due to the large variability found between different sensors, the 28 force sensors used on the final prototype of the insoles were calibrated individually to increase the accuracy of the measurements.

The preliminary experiments of the insole showed better results when the insole was tested against a rigid surface such as the floor (9.86\% of error), compared to the results obtained once it was placed inside the shoe (20.02\% of error) during single stance static experiments. This can be due to the more compliant materials inside the shoe, which allows for indentation and deflections. In the other hand, better results were obtained during dynamic testing (walking experiments) compared with static test, with errors of $7.85 \%$ and $10.64 \%$ for the dynamic and static experiments respectively. Despite the errors on the scaling, we obtained high correlation values (above 0.95) between the insole and the force plate recording for the VGRF. In terms

of the COP, the results from the walking experiments of the final insole prototype showed mean correlation value of 0.99 and 0.84 , and average errors of $2.72 \mathrm{~mm}$ and $3.5 \mathrm{~mm}$ for the $\mathrm{A} / \mathrm{P}$ and $\mathrm{M} / \mathrm{L}$ direction respectively. 


\section{Estimation of shear forces using ANN}

The preliminary results on Chapter 5 showed the feasibility of estimating the shear

components of the ground reaction force from the distribution of the forces during walking using artificial neural networks. This methodology was later implemented on the experiments with healthy subjects in Chapter 6 .

\section{Experiments with healthy subjects}

The results from the standing and walking experiments with seven healthy subjects showed the capability of the insole to measure the changes and variations on the COP on different stability conditions as well as during locomotion. Furthermore, the insole was able to measure the VRGF within $7 \%$ error and the method presented for estimating the shear components of the GRF was shown to yield errors less than $15 \%$ using data from the same subject. The presented measurement strategy then seems suitable for application in the overall BalanceAid system.

\subsection{Future Work}

As the first prototype of this sensor the results from experiments seemed satisfactory. However, there are some improvements that can be implemented for a next iteration of the device.

\section{Data acquisition improvements}

In terms of the electronic components, the electronic case containing the circuits for the conditioning of the signals from the force sensors can be replaced with a small printed circuit board. This way, we can reduce its size and weight and mount it near the ADC on the Raspberry Pi to reduce wiring. Additionally, alternative solutions 
for the analog to digital conversion can be implemented to obtain a faster sampling rate.

\section{Sensor modifications}

Another improvement to the system is to build a more robust prototype of the insole. Currently, adhesive taped is being used to keep the two insole layers together. However, long-term use of the insole could cause the parts to move and create a misalignment on the puck areas. Hence, the interface with the sensors and their output readings could be modified. To prevent this, alternative methods could be implemented to better hold both layers of the insole together.

To overcome some of the problems encountered when the insole was placed inside the shoe an outsole version could be created. This version could be attached to the sole of the shoes, while remaining flexible and thin. Thereupon, by having a more rigid interface with the sensor, the measurements would be more consistent. Moreover, the temperature dependencies of the sensor could be also investigated, as well as the long term repeatability.

\section{Shear forces estimation}

Future work should look at the static performance of the ANN estimator. Additional experiments could be performed to include different motions during the training of the ANN. Also, other inputs may be added in the estimation to improve the performance of the network in different scenarios, such as a measure of the horizontal acceleration of the body.

\section{BalanceAid device integration}

Finally, future work consists on full integration of the insole measurements with the BalanceAid device and the choice of balance parameters for tactile feedback to the 
user. Therefore, with the early experiments with the integrated system, one can suggest whether further improvement of the sensor accuracy is needed or not. 


\section{List of References}

[1] N. Hayafune, Y. Hayafune, and H. Jacob. "Pressure and force distribution characteristics under the normal foot during the push-off phase in gait." The Foot 9(2), 88-92 (1999).

[2] A. Putti, G. Arnold, and R. Abboud. "Foot pressure differences in men and women." Foot and ankle surgery 16(1), 21-24 (2010).

[3] J. Rose and J. Gamble. Human walking. Williams \& Wilkins. ISBN 9780683073607 (1994).

[4] G. Giakas and V. Baltzopoulos. "Time and frequency domain analysis of ground reaction forces during walking: an investigation of variability and symmetry." Gait \& Posture 5(3), 189-197 (1997).

[5] C. Kirtley. Clinical gait analysis: theory and practice. Elsevier, New York; Edinburgh. ISBN 9780443100093; 0443100098 (2006).

[6] D. A. Winter. "Human balance and posture control during standing and walking." Gait \& posture 3(4), 193-214 (1995).

[7] Bertec. Bertec Force Plates. www.bertec.com (2012).

[8] S. J. M. Bamberg, A. Y. Benbasat, D. M. Scarborough, D. E. Krebs, and J. A. Paradiso. "Gait analysis using a shoe-integrated wireless sensor system." Information Technology in Biomedicine, IEEE Transactions on 12(4), 413-423 (2008).

[9] A. Howell, T. Kobayashi, H. Hayes, K. Foreman, and S. Bamberg. "Kinetic gait analysis using a low-cost insole." IEEE transactions on bio-medical engineering 60(12), 3284-3290 (2013).

[10] T. Liu, Y. Inoue, and K. Shibata. "A wearable force plate system for the continuous measurement of triaxial ground reaction force in biomechanical applications." Measurement Science and Technology 21(8), 085804 (2010). 
[11] Tekscan. FlexiForce Sensors User Manual. www.tekscan.com.

[12] Smooth-on. "Durometer shore hardness scale." (2008).

[13] W. Hutton and M. Dhanendran. "A study of the distribution of load under the normal foot during walking." International orthopaedics 3(2), 153-157 (1979).

[14] W. R. Ledoux and H. J. Hillstrom. "The distributed plantar vertical force of neutrally aligned and pes planus feet." Gait \& posture 15(1), 1-9 (2002).

[15] Vestibular Disorders Association. "The human balance system."

[16] Y. Agrawal, J. Carey, C. Della Santina, M. Schubert, and L. Minor. "Disorders of balance and vestibular function in US adults: data from the National Health and Nutrition Examination Survey, 2001-2004." Arch Intern Med 169(10), 938-944 (2009).

[17] National Institute on Deafness and Other Communication Disorders (NIDCD). "Strategic Plan (FY 2006-2008)." (2014).

[18] C. Hobeika. "Equilibrium and balance in the elderly." Ear Nose Throat J 78(8), 558-62, 565-6 (1999).

[19] Public Health Agency of Canada. "The facts: Seniors and Injury in Canada." http://www.phac-aspc.gc.ca/seniors-aines/publications/ public/injury-blessure/safelive-securite/chap2-eng.php (2011).

[20] P. Bach-y Rita and S. W Kercel. "Sensory substitution and the human-machine interface." Trends in cognitive sciences 7(12), 541-546 (2003).

[21] N. Vuillerme, P. Hlavackova, C. Franco, B. Diot, J. Demongeot, and Y. Payan. "Can an electro-tactile vestibular substitution system improve balance in patients with unilateral vestibular loss under altered somatosensory conditions from the foot and ankle?" In "Engineering in Medicine and Biology Society, EMBC, 2011 Annual International Conference of the IEEE," pages 1323-1326. IEEE (2011).

[22] M. Tyler, Y. Danilov, and P. Bach-y Rita. "Closing an open-loop control system: vestibular substitution through the tongue." Journal of integrative neuroscience 2(02), 159-164 (2003).

[23] C. Wall, M. S. Weinberg, P. B. Schmidt, and D. E. Krebs. "Balance prosthesis based on micromechanical sensors using vibrotactile feedback of tilt." Biomedical Engineering, IEEE Transactions on 48(10), 1153-1161 (2001). 
[24] C. Wall III, D. M. Wrisley, and K. D. Statler. "Vibrotactile tilt feedback improves dynamic gait index: a fall risk indicator in older adults." Gait $\&$ posture $\mathbf{3 0}(1)$, 16-21 (2009).

[25] K. H. Sienko, V. V. Vichare, M. D. Balkwill, and C. Wall. "Assessment of vibrotactile feedback on postural stability during pseudorandom multidirectional platform motion." Biomedical Engineering, IEEE Transactions on 57(4), 944$952(2010)$.

[26] S. Haggerty, L.-T. Jiang, A. Galecki, and K. H. Sienko. "Effects of biofeedback on secondary-task response time and postural stability in older adults." Gait \& posture 35(4), 523-528 (2012).

[27] T. E. Prieto, J. Myklebust, R. Hoffmann, E. Lovett, and B. Myklebust. "Measures of postural steadiness: differences between healthy young and elderly adults." Biomedical Engineering, IEEE Transactions on 43(9), 956-966 (1996).

[28] M. de Haart, A. C. Geurts, S. C. Huidekoper, L. Fasotti, and J. van Limbeek. "Recovery of standing balance in postacute stroke patients: a rehabilitation cohort study." Archives of physical medicine and rehabilitation 85(6), 886-895 (2004).

[29] M. Dozza, L. Chiari, and F. B. Horak. "Audio-biofeedback improves balance in patients with bilateral vestibular loss." Archives of physical medicine and rehabilitation 86(7), 1401-1403 (2005).

[30] X. Tortolero, K. Masani, T. A. Thrasher, and M. R. Popovic. "Instability prediction by monitoring center of pressure during standing." In "Engineering in Medicine and Biology Society, 2006. EMBS'06. 28th Annual International Conference of the IEEE," pages 5412-5415. IEEE (2006).

[31] A. Karlsson and G. Frykberg. "Correlations between force plate measures for assessment of balance." Clinical Biomechanics 15(5), 365-369 (2000).

[32] J. Raymakers, M. Samson, and H. Verhaar. "The assessment of body sway and the choice of the stability parameter (s)." Gait \& posture 21(1), 48-58 (2005).

[33] M. R. Popović, I. P. Pappas, K. Nakazawa, T. Keller, M. Morari, and V. Dietz. "Stability criterion for controlling standing in able-bodied subjects." Journal of Biomechanics 33(11), 1359-1368 (2000). 
[34] M. B. Popovic, A. Goswami, and H. Herr. "Ground reference points in legged locomotion: Definitions, biological trajectories and control implications." The International Journal of Robotics Research 24(12), 1013-1032 (2005).

[35] S. J. M. Bamberg, P. LaStayo, L. Dibble, J. Musselman, and S. K. D. Raghavendra. "Development of a quantitative in-shoe measurement system for assessing balance: sixteen-sensor insoles." In "Engineering in Medicine and Biology Society, 2006. EMBS'06. 28th Annual International Conference of the IEEE," pages 6041-6044. IEEE (2006).

[36] L. S. Lincoln and S. J. M. Bamberg. "Insole sensor system for real-time detection of biped slip." In "Engineering in Medicine and Biology Society (EMBC), 2010 Annual International Conference of the IEEE," pages 1449-1452. IEEE (2010).

[37] P. S. Dyer and S. J. M. Bamberg. "Instrumented insole vs. force plate: A comparison of center of plantar pressure." In "Engineering in Medicine and Biology Society, EMBC, 2011 Annual International Conference of the IEEE," pages 6805-6809. IEEE (2011).

[38] P. H. Veltink, C. Liedtke, E. Droog, and H. van der Kooij. "Ambulatory measurement of ground reaction forces." Neural Systems and Rehabilitation Engineering, IEEE Transactions on 13(3), 423-427 (2005).

[39] C. Liedtke, S. A. Fokkenrood, J. T. Menger, H. van der Kooij, and P. H. Veltink. "Evaluation of instrumented shoes for ambulatory assessment of ground reaction forces." Gait \& posture 26(1), 39-47 (2007).

[40] A. Catteau. A Wearable Computer Vision System for Measuring Shoe Position and Orientation. Master's thesis, Carleton University, Ottawa (2014).

[41] K. Berg, S. Wood-Dauphinee, J. Williams, and B. Maki. "Measuring balance in the elderly: validation of an instrument." Canadian journal of public health= Revue canadienne de santé publique 83, S7 (1992).

[42] A. M. Myers, P. C. Fletcher, A. H. Myers, and W. Sherk. "Discriminative and evaluative properties of the activities-specific balance confidence (abc) scale." The Journals of Gerontology Series A: Biological Sciences and Medical Sciences 53(4), M287-M294 (1998).

[43] M. Tinetti. "Performance-oriented assessment of mobility problems in elderly patients." Journal of the American Geriatrics Society 34(2), 119 (1986). 
[44] S. Mathias, U. Nayak, and B. Isaacs. "Balance in elderly patients: the" get-up and go" test." Archives of physical medicine and rehabilitation 67(6), 387-389 (1986).

[45] P. W. Duncan, D. K. Weiner, J. Chandler, and S. Studenski. "Functional reach: a new clinical measure of balance." Journal of gerontology 45(6), M192-M197 (1990).

[46] M. Mancini and F. B. Horak. "The relevance of clinical balance assessment tools to differentiate balance deficits." European journal of physical and rehabilitation medicine 46(2), 239 (2010).

[47] K. Sienko, M. Balkwill, L. Oddsson, and C. Wall. "Effects of multi-directional vibrotactile feedback on vestibular-deficient postural performance during continuous multi-directional support surface perturbations." Journal of Vestibular Research 18(5), 273-285 (2008).

[48] A. M. Howell, T. Kobayashi, T. R. Chou, W. Daly, M. Orendurff, and S. J. M. Bamberg. "A laboratory insole for analysis of sensor placement to determine ground reaction force and ankle moment in patients with stroke." In "Engineering in Medicine and Biology Society (EMBC), 2012 Annual International Conference of the IEEE," pages 6394-6397. IEEE (2012).

[49] A. M. Howell. Insole-based gait analysis. Master's thesis, The University of Utah (2012).

[50] S. J. Morris. A shoe-integrated sensor system for wireless gait analysis and realtime therapeutic feedback. Ph.D. thesis, Massachusetts Institute of Technology (2004).

[51] J. Hausdorff, L. Zemany, C.-K. Peng, and A. Goldberger. "Maturation of gait dynamics: stride-to-stride variability and its temporal organization in children." Journal of Applied Physiology 86(3), 1040-1047 (1999).

[52] M. Roland, J. E. Mark, B. Erman, and K. (Firm). The science and technology of rubber. Academic Press, Boston; Amsterdam. ISBN 9780123945846; 0123948320; 9780123948328; 0123945844 (2013).

[53] E. K. Antonsson and R. W. Mann. "The frequency content of gait." Journal of biomechanics 18(1), 39-47 (1985). 
[54] H. Savelberg and A. De Lange. "Assessment of the horizontal, fore-aft component of the ground reaction force from insole pressure patterns by using artificial neural networks." Clinical Biomechanics 14(8), 585-592 (1999).

[55] A. Forner Cordero, H. Koopman, and F. Van Der Helm. "Use of pressure insoles to calculate the complete ground reaction forces." Journal of biomechanics $\mathbf{3 7}(9)$, 1427-1432 (2004).

[56] D. T.-P. Fong, Y.-Y. Chan, Y. Hong, P. S.-H. Yung, K.-Y. Fung, and K.-M. Chan. "Estimating the complete ground reaction forces with pressure insoles in walking." Journal of biomechanics 41(11), 2597-2601 (2008).

[57] H. Rouhani, J. Favre, X. Crevoisier, and K. Aminian. "Ambulatory assessment of $3 \mathrm{~d}$ ground reaction force using plantar pressure distribution." Gait $\&$ posture 32(3), 311-316 (2010).

[58] G. Dreyfus. Neural networks: methodology and applications. Springer, New York; Berlin. ISBN $9783540229803 ; 3540229809$ (2005).

[59] P. B. Davenport. A vector approach to the algebra of rotations with applications. National Aeronautics and Space Administration (1968). 


\section{Appendix A}

\section{Ethics Clearance Form}

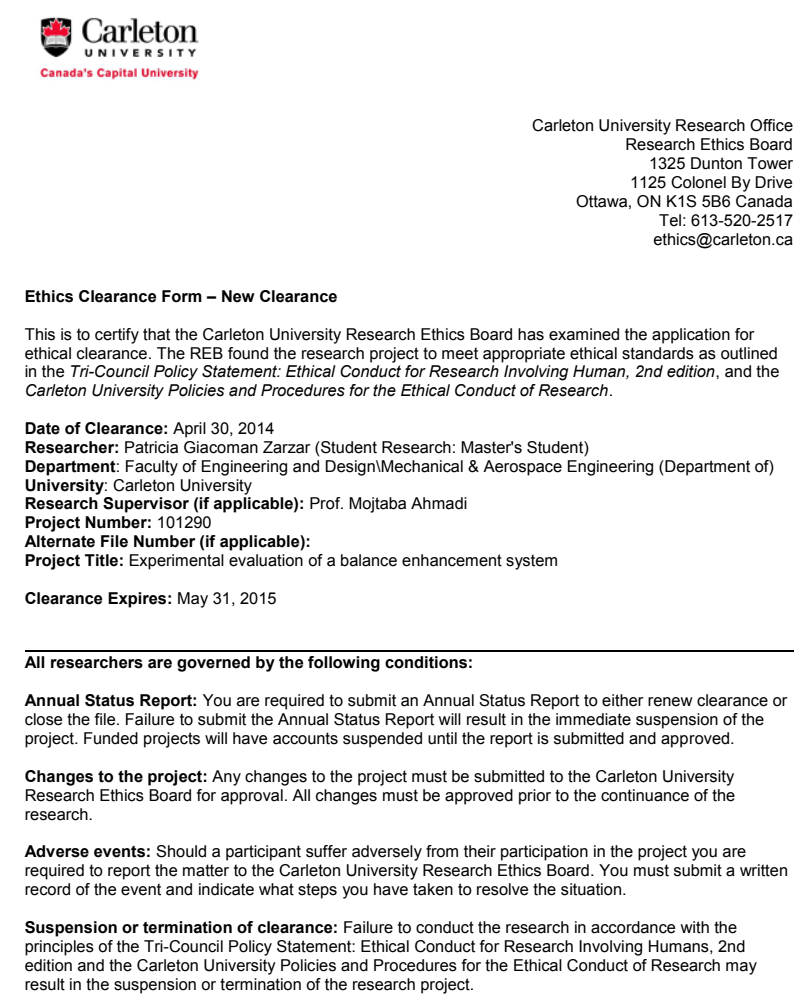

Figure A.1: Carleton University Ethics Clearance Form 


\section{Appendix B}

\section{Conditioning circuit diagram}

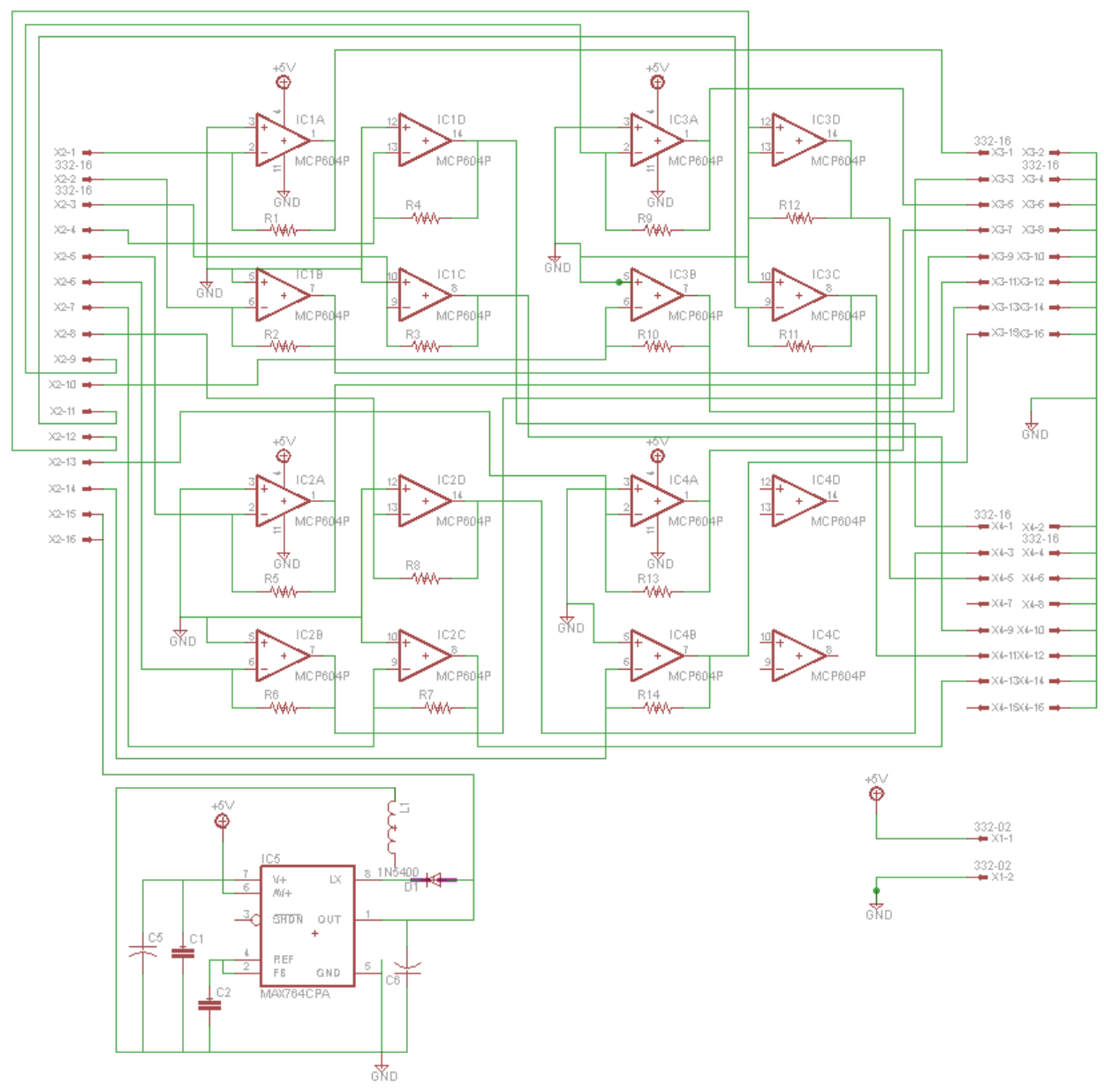

Figure B.1: Schematic diagram of electronic circuit for conditioning signals of force sensors. 


\section{Appendix C}

\section{Coordinate System Transformation}

\section{Method}

To calculate the transformation matrix from the different coordinate systems, the solution provided by Davenport to solve Wahbas optimization problem was used [59].

For this method, 3 points are required to be known on both coordinate systems in order to calculate the transformation matrix. The objective is to find the orthogonal matrix A that minimize Wahba's loss function

$$
L(A)=\frac{1}{2} \sum_{i=1}^{n} a_{i}\left|b_{i}-A r_{i}\right|^{2}
$$

In order to do this, the Davenport's q-method converts the Wahba's loss function in the quadratic form

$$
q^{T} K q
$$

where $q^{T}$ is a rotational quaternion that brings $r_{i}$ to $b_{i}$ and $K$ is a $4 \times 4$ matrix given by

$$
K=\left(\begin{array}{cc}
\sigma & Z^{T} \\
Z & S-\sigma I_{3}
\end{array}\right)
$$


where

$$
\begin{gathered}
Z=\left(\begin{array}{c}
B_{2} 3-B_{3} 2 \\
B_{3} 1-B_{1} 3 \\
B_{1} 2-B_{2} 1
\end{array}\right) \\
S=B+B^{T} \\
\sigma=\operatorname{trace}(B) \\
B=\sum_{n} v_{n b} v_{n a}^{T} \\
K q^{T}=\lambda q
\end{gathered}
$$

The problem is then reduced to finding the eigenvector with the largest eigenvalue of $K$. 


\section{Appendix D}

\section{Additional Experiments Results}

The Figures D.1 - D.5 show the variation on the COP in the 1 foot standing experiments from Section 6.1 for the remaining subjects.

The Figures D.6 - D.11 show the results from a sample step from each of the subjects during the walking experiments from Section 6.2. 


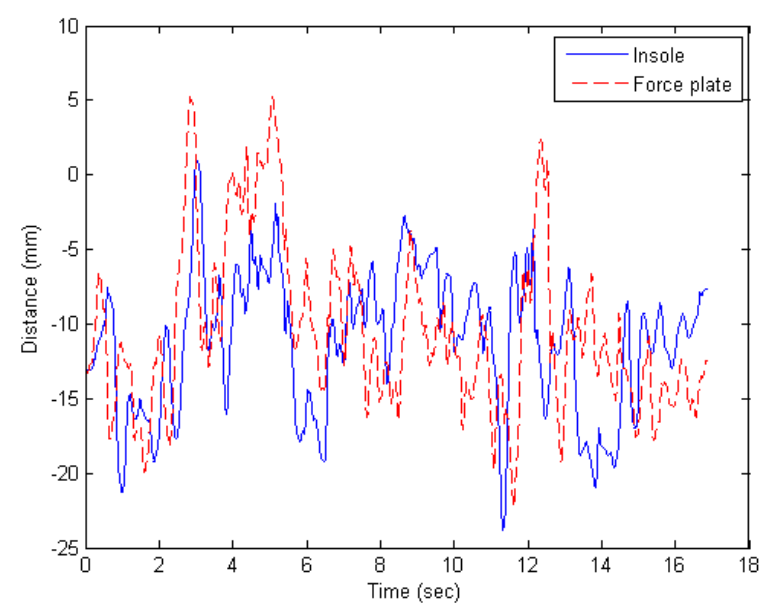

(a) $\mathrm{COP}$ in the $\mathrm{A} / \mathrm{P}$ direction

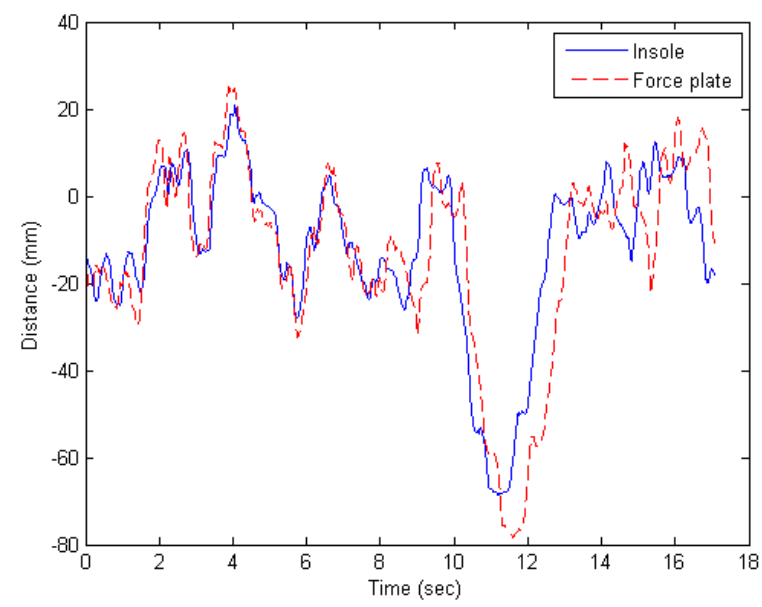

(c) $\mathrm{COP}$ in the $\mathrm{A} / \mathrm{P}$ direction

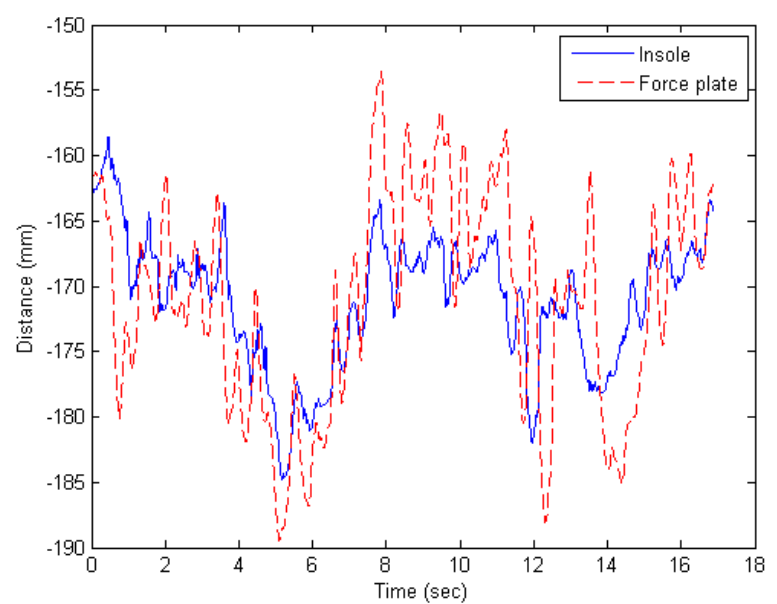

(b) $\mathrm{COP}$ in the $\mathrm{M} / \mathrm{L}$ direction

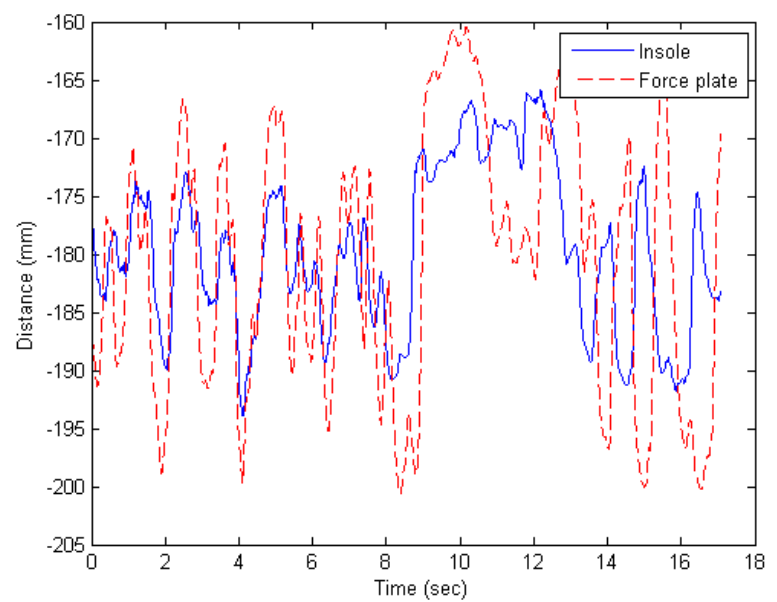

(d) COP in the M/L direction

Figure D.1: Comparison of insole (blue) and force plate (red) measurements of COP from subject 2 standing on one foot with eyes opened (a), (b) and with eyes closed (c), (d). 


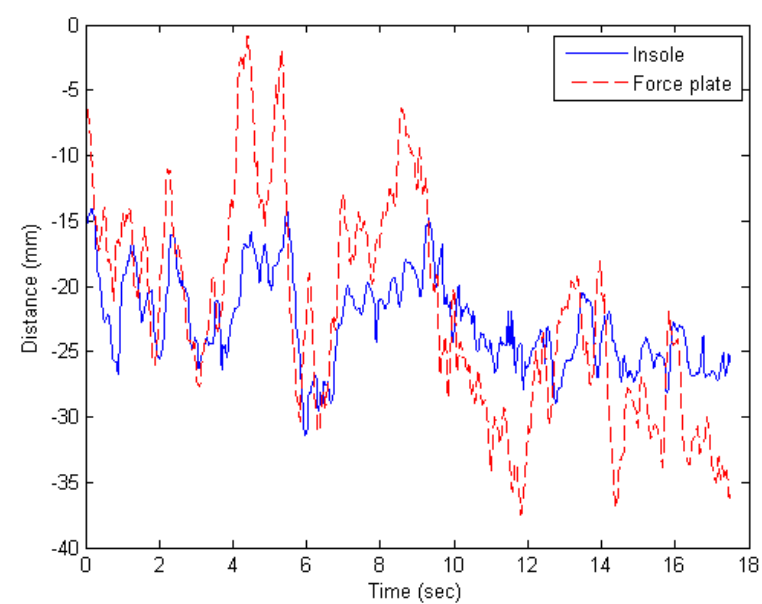

(a) $\mathrm{COP}$ in the $\mathrm{A} / \mathrm{P}$ direction

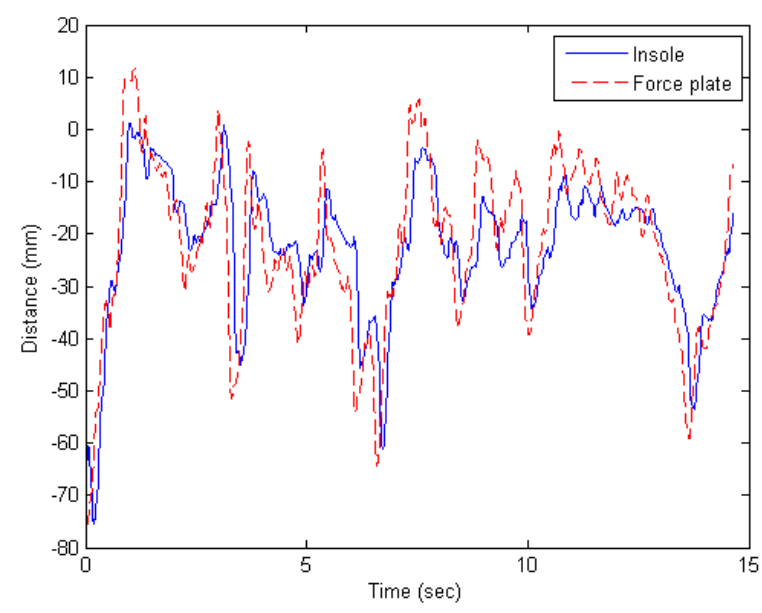

(c) $\mathrm{COP}$ in the $\mathrm{A} / \mathrm{P}$ direction

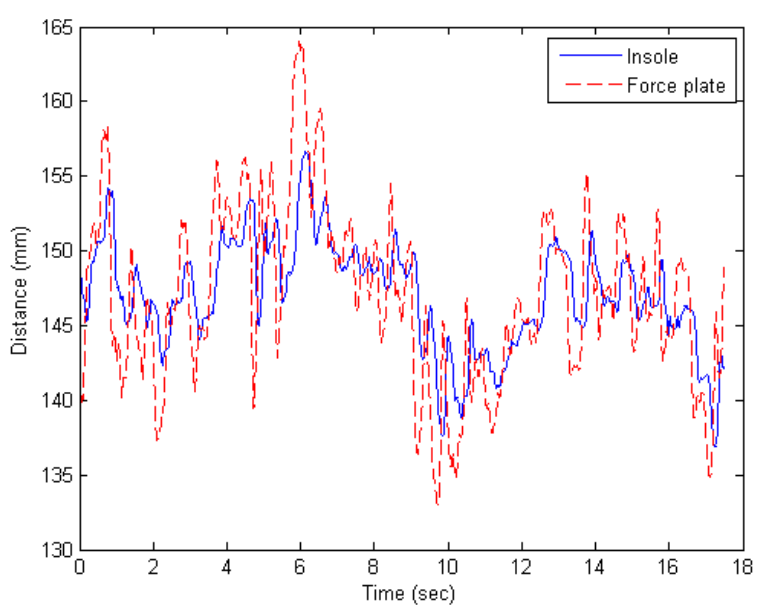

(b) $\mathrm{COP}$ in the $\mathrm{M} / \mathrm{L}$ direction

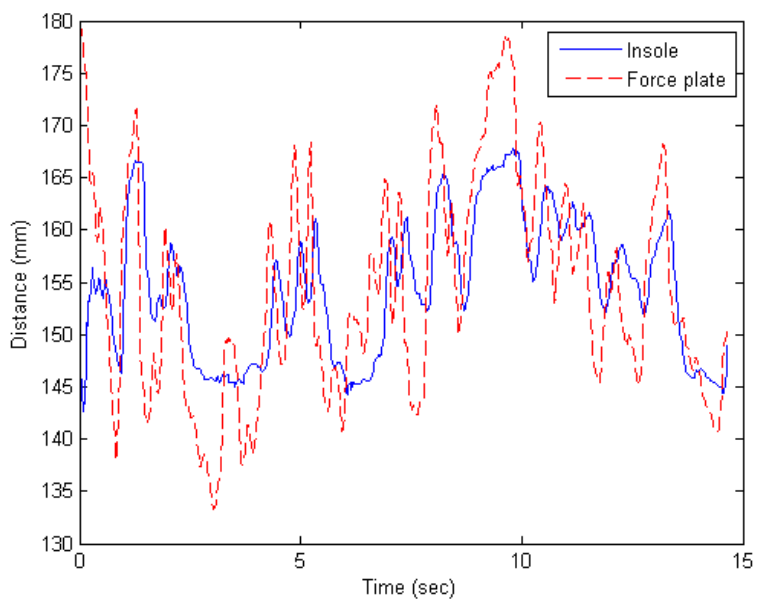

(d) COP in the M/L direction

Figure D.2: Comparison of insole (blue) and force plate (red) measurements of COP from subject 3 standing on one foot with eyes opened (a), (b) and with eyes closed (c), (d). 


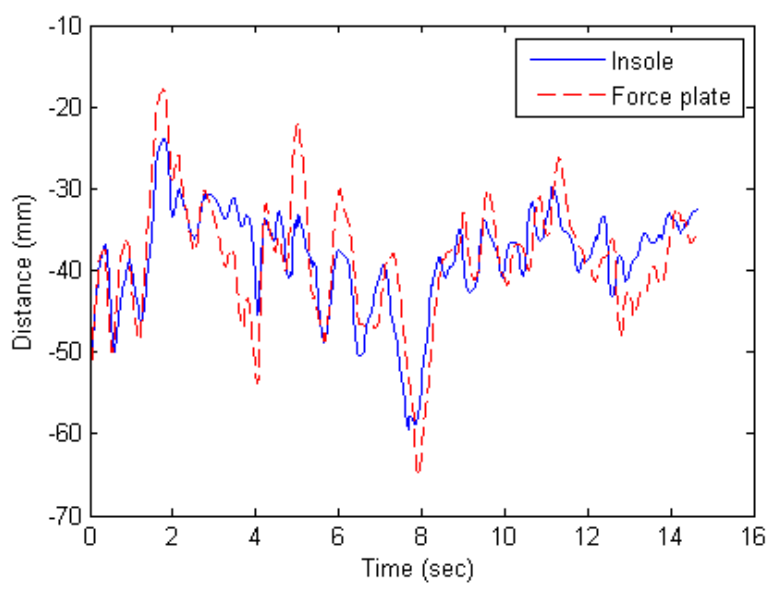

(a) $\mathrm{COP}$ in the $\mathrm{A} / \mathrm{P}$ direction

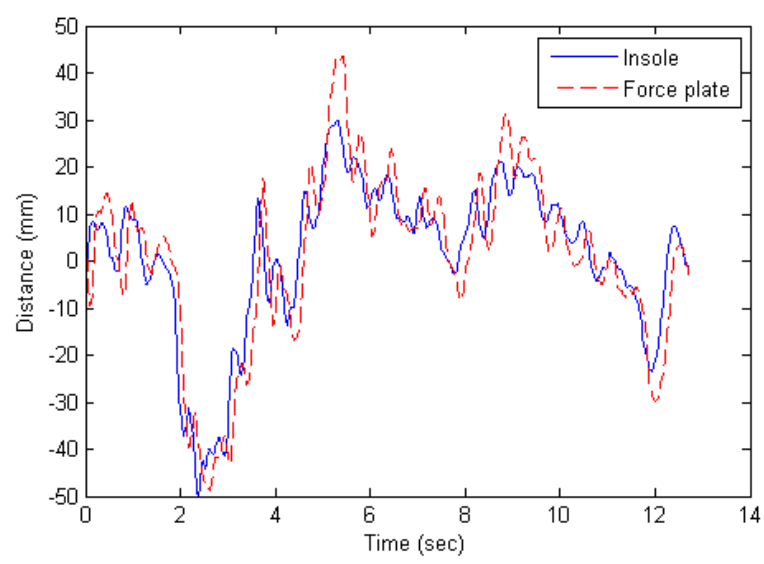

(c) $\mathrm{COP}$ in the $\mathrm{A} / \mathrm{P}$ direction

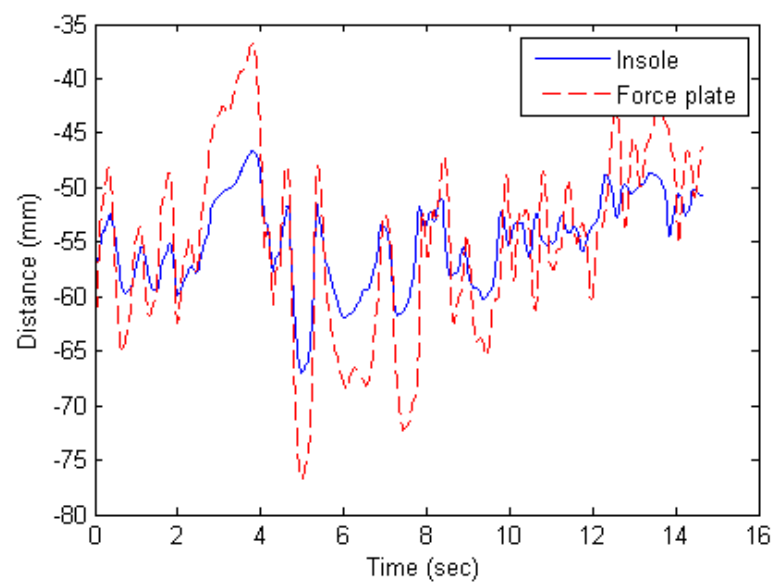

(b) $\mathrm{COP}$ in the $\mathrm{M} / \mathrm{L}$ direction

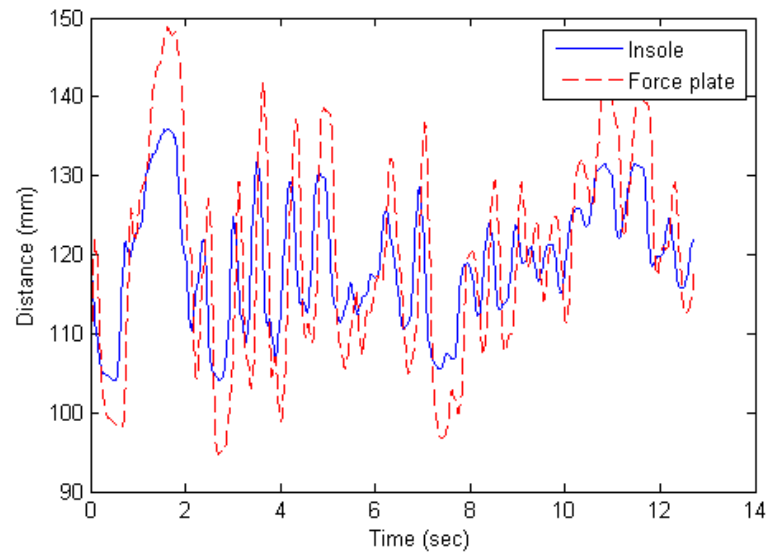

(d) COP in the M/L direction

Figure D.3: Comparison of insole (blue) and force plate (red) measurements of COP from subject 4 standing on one foot with eyes opened (a), (b) and with eyes closed (c), (d). 


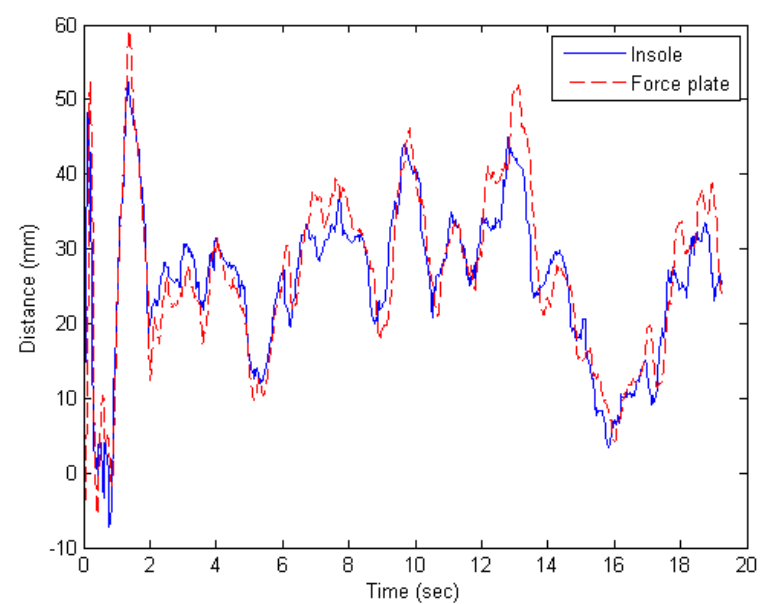

(a) $\mathrm{COP}$ in the $\mathrm{A} / \mathrm{P}$ direction

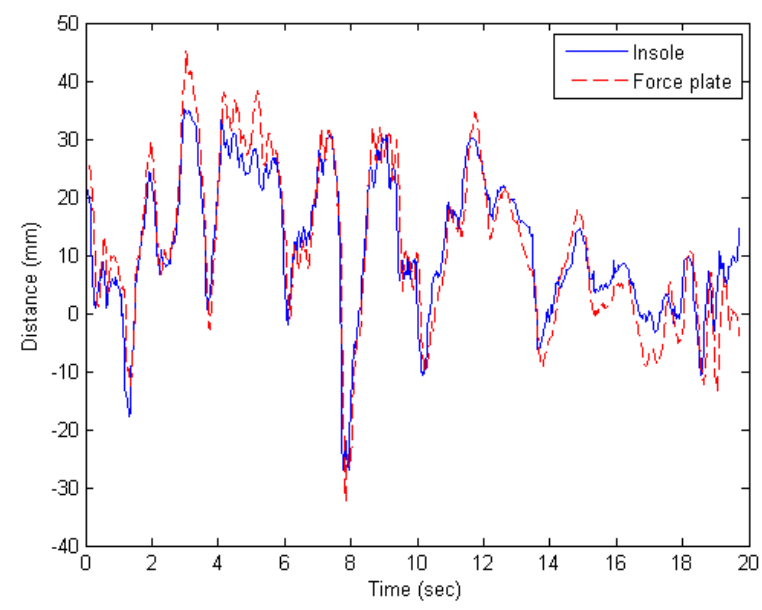

(c) $\mathrm{COP}$ in the $\mathrm{A} / \mathrm{P}$ direction

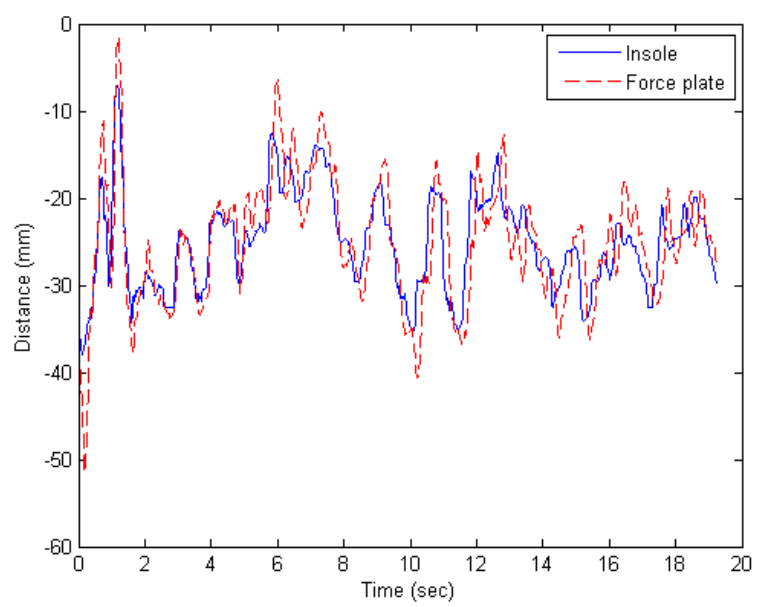

(b) $\mathrm{COP}$ in the $\mathrm{M} / \mathrm{L}$ direction

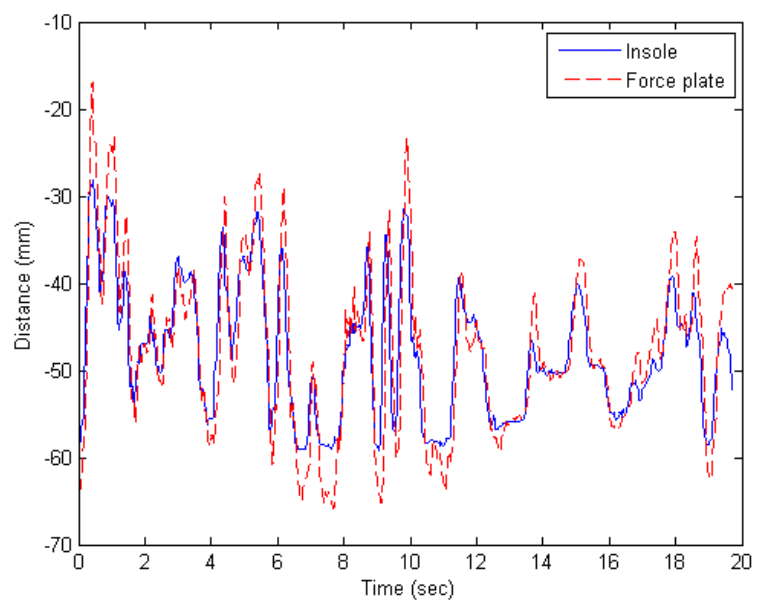

(d) COP in the M/L direction

Figure D.4: Comparison of insole (blue) and force plate (red) measurements of COP from subject 5 standing on one foot with eyes opened (a), (b) and with eyes closed (c), (d). 


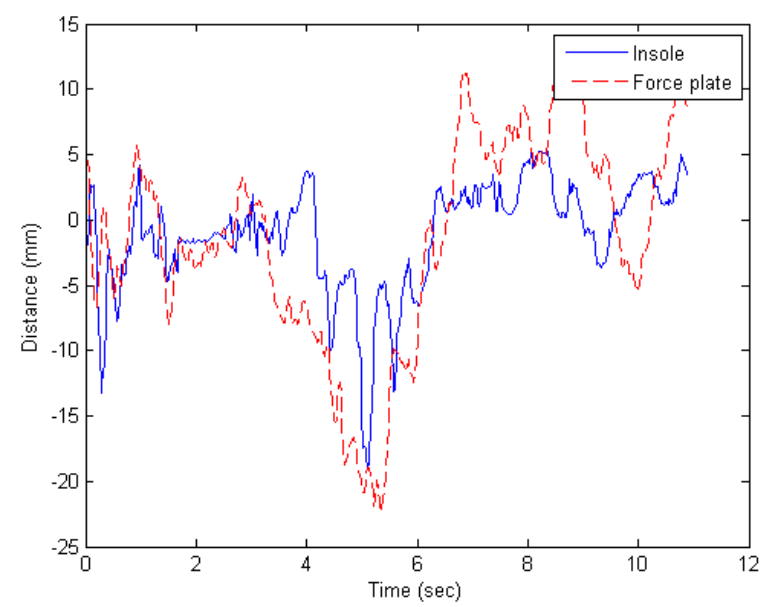

(a) $\mathrm{COP}$ in the $\mathrm{A} / \mathrm{P}$ direction

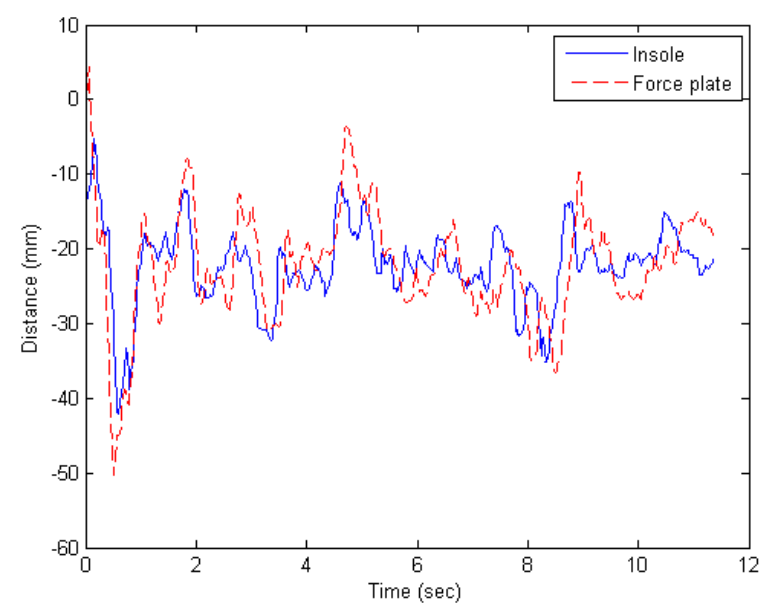

(c) $\mathrm{COP}$ in the $\mathrm{A} / \mathrm{P}$ direction

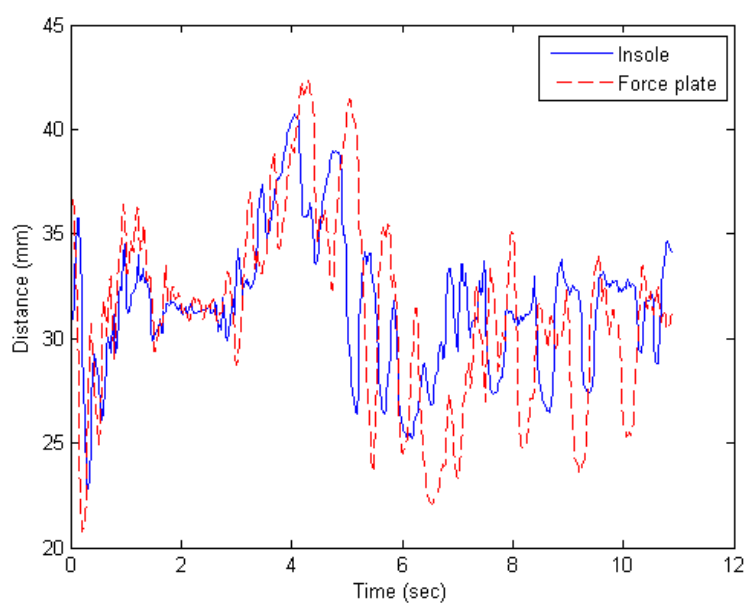

(b) $\mathrm{COP}$ in the $\mathrm{M} / \mathrm{L}$ direction

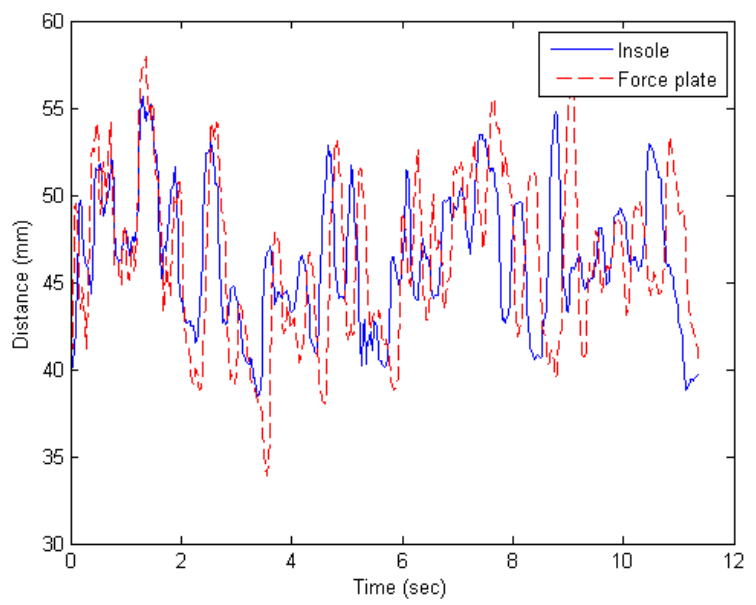

(d) COP in the M/L direction

Figure D.5: Comparison of insole (blue) and force plate (red) measurements of COP from subject 7 standing on one foot with eyes opened (a), (b) and with eyes closed (c), (d). 


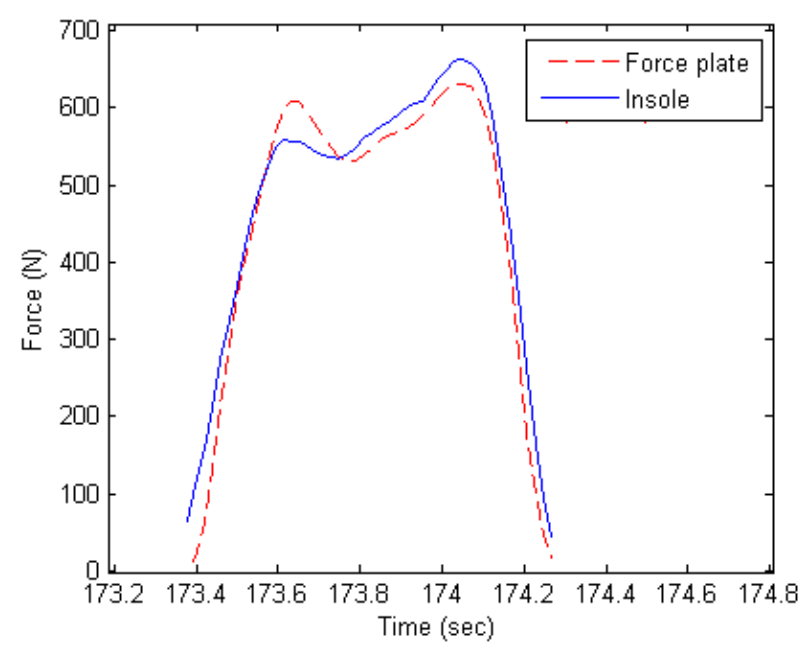

(a) VGRF

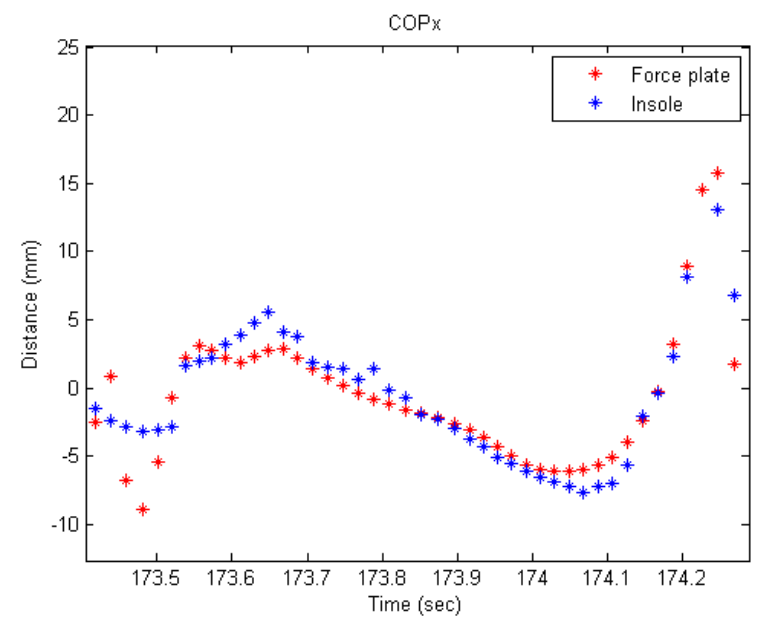

(c) $\mathrm{COPx}$

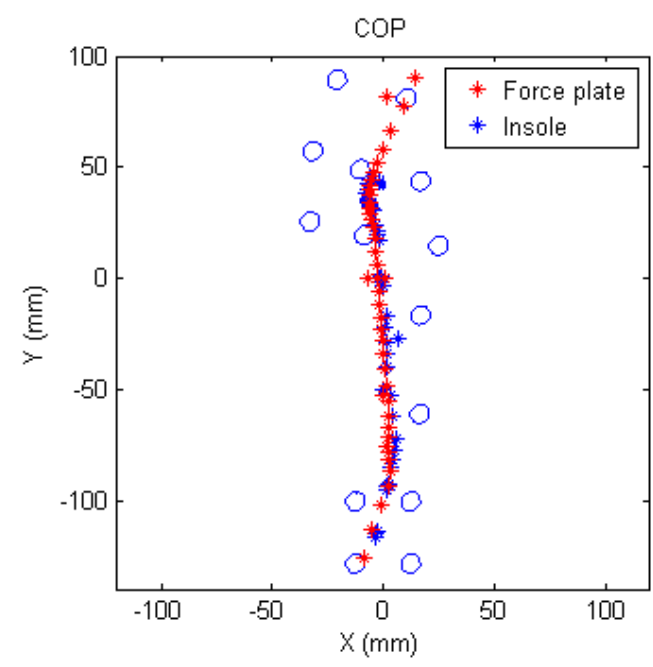

(b) COP

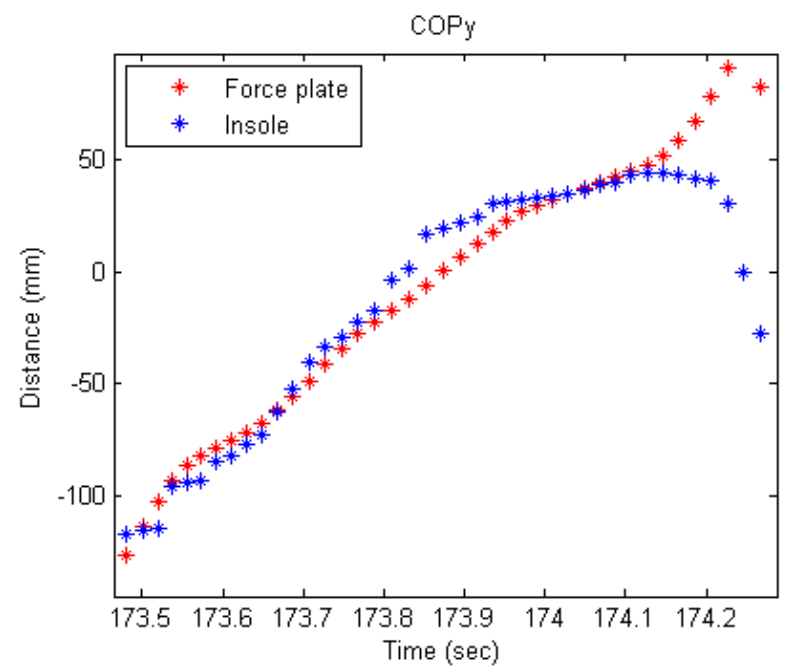

(d) $\mathrm{COPy}$

Figure D.6: Comparison of insole (blue) and force plate (red) measurements of GRF and COP for one example step of the walking experiments from subject 1. 


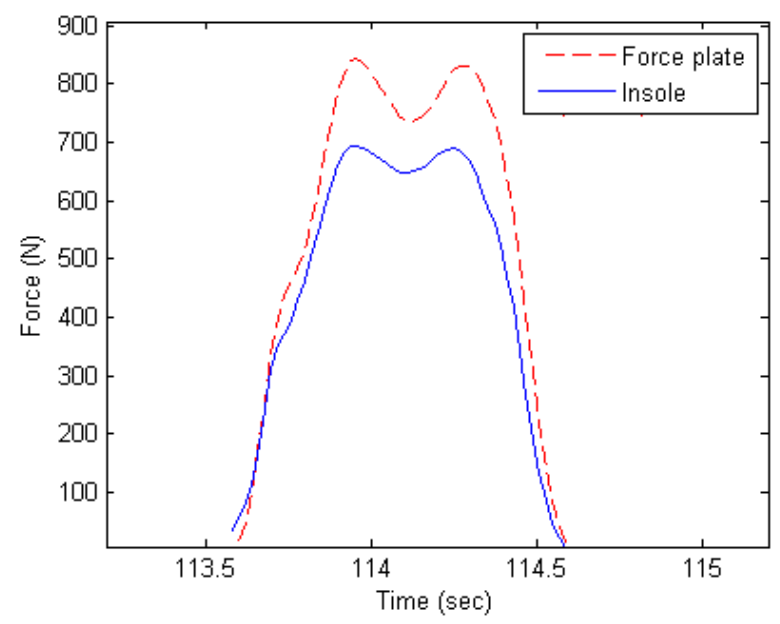

(a) VGRF

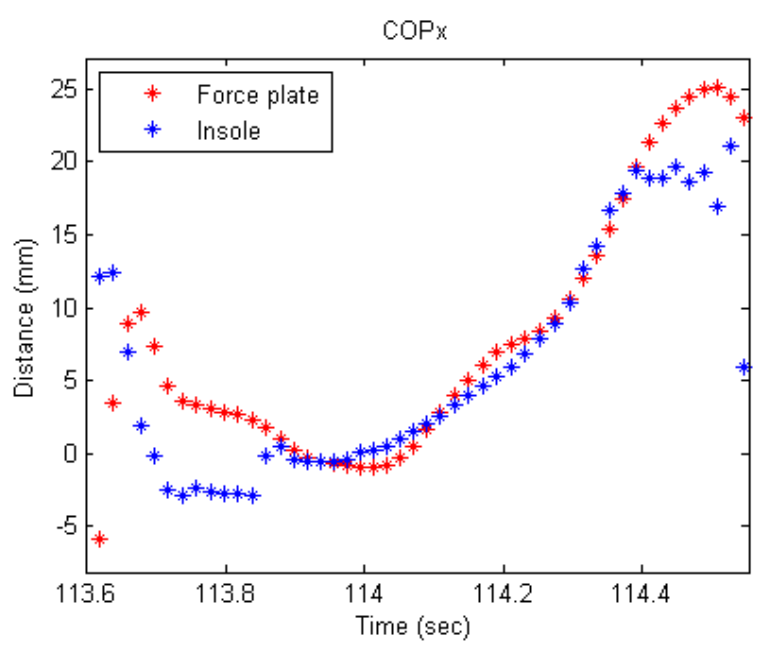

(c) $\mathrm{COPx}$

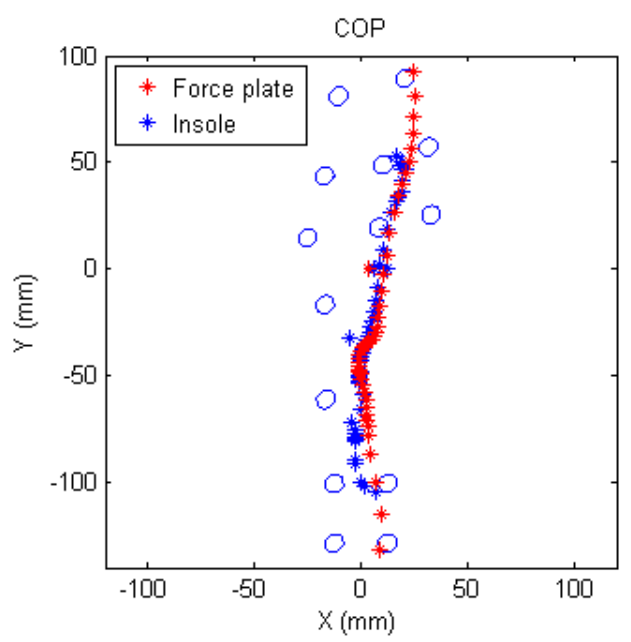

(b) COP

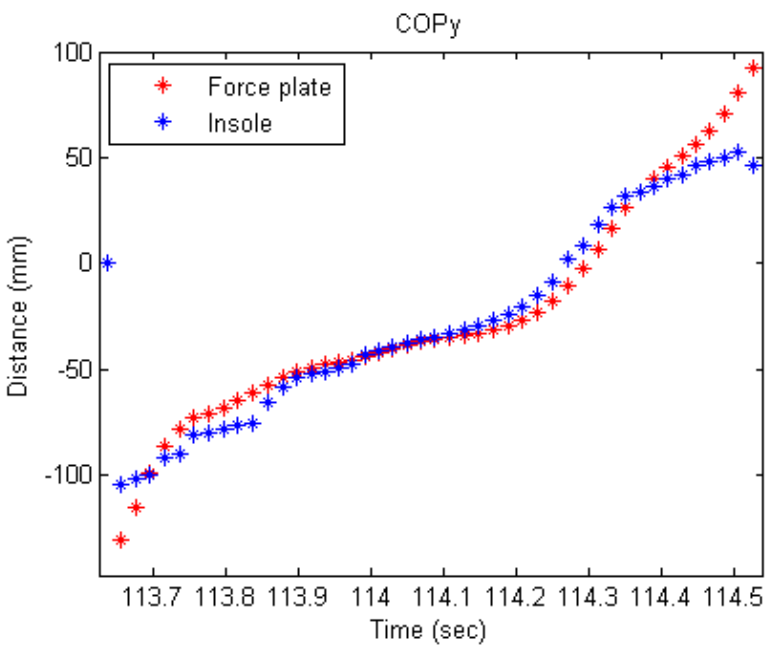

(d) $\mathrm{COPy}$

Figure D.7: Comparison of insole (blue) and force plate (red) measurements of GRF and COP for one example step of the walking experiments from subject 2 . 


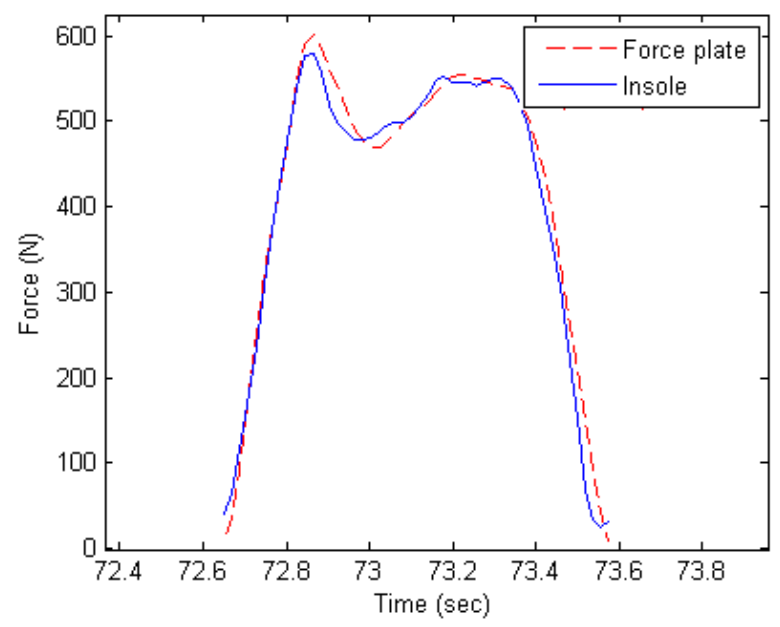

(a) VGRF

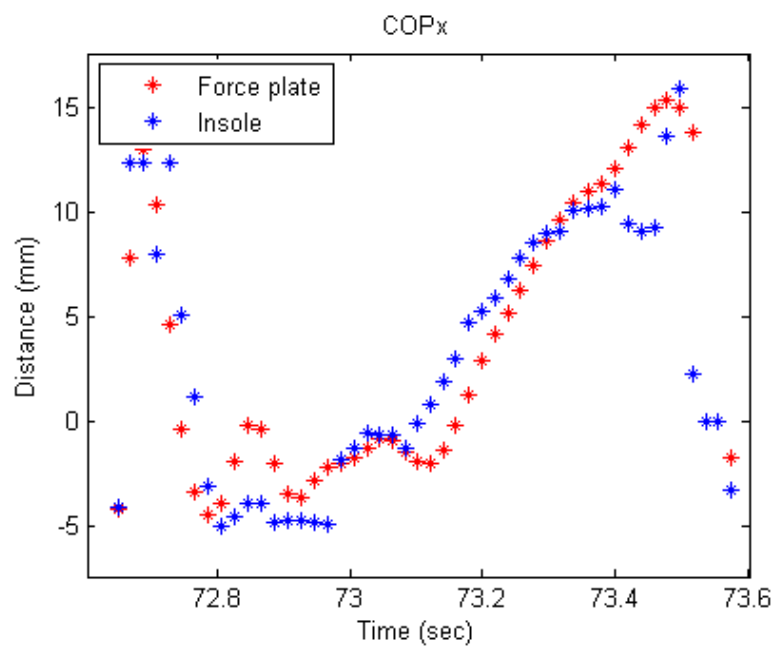

(c) $\mathrm{COPx}$

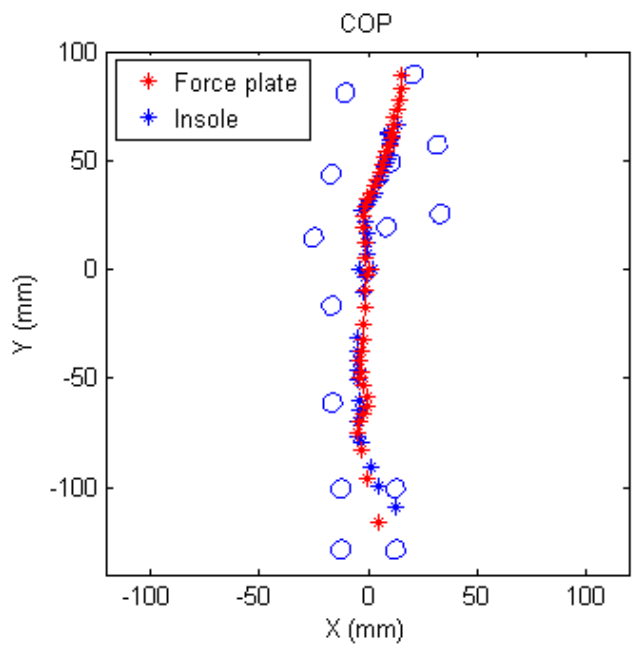

(b) COP

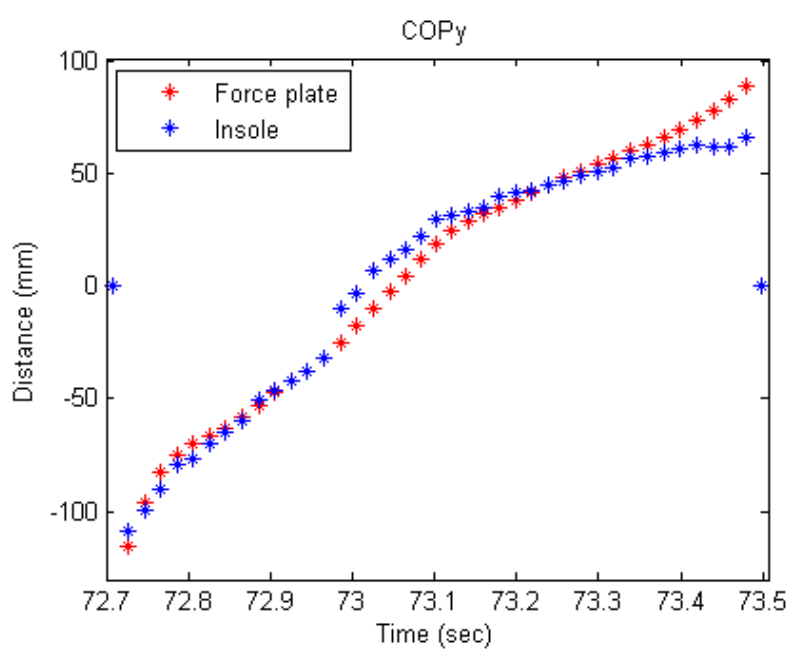

(d) $\mathrm{COPy}$

Figure D.8: Comparison of insole (blue) and force plate (red) measurements of GRF and COP for one example step of the walking experiments from subject 3 . 


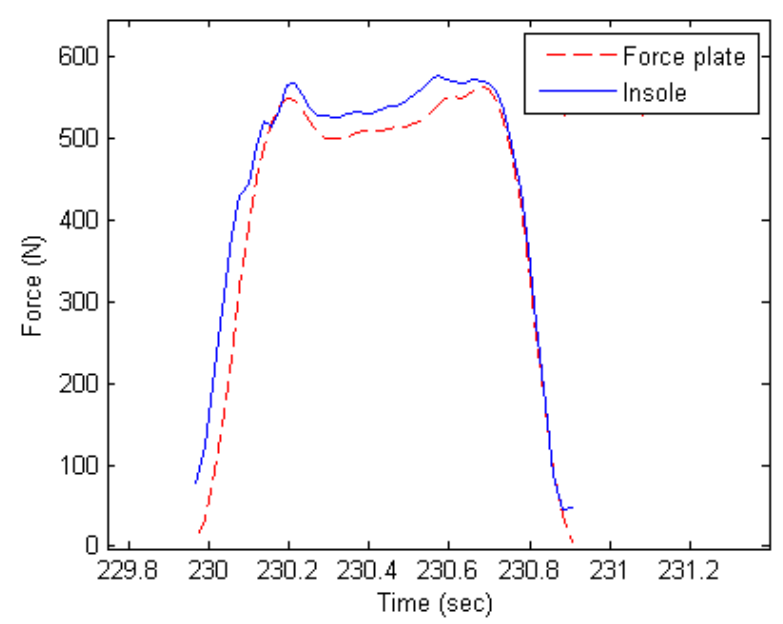

(a) VGRF

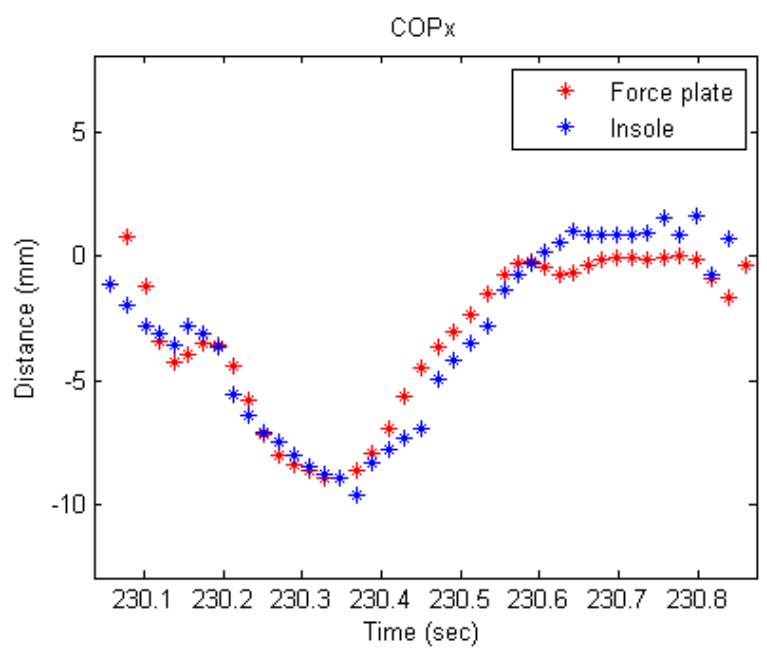

(c) $\operatorname{COPx}$

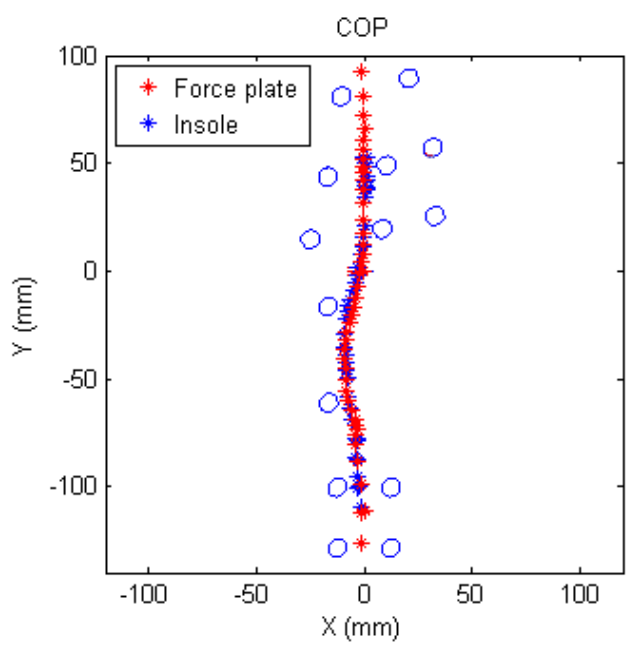

(b) COP

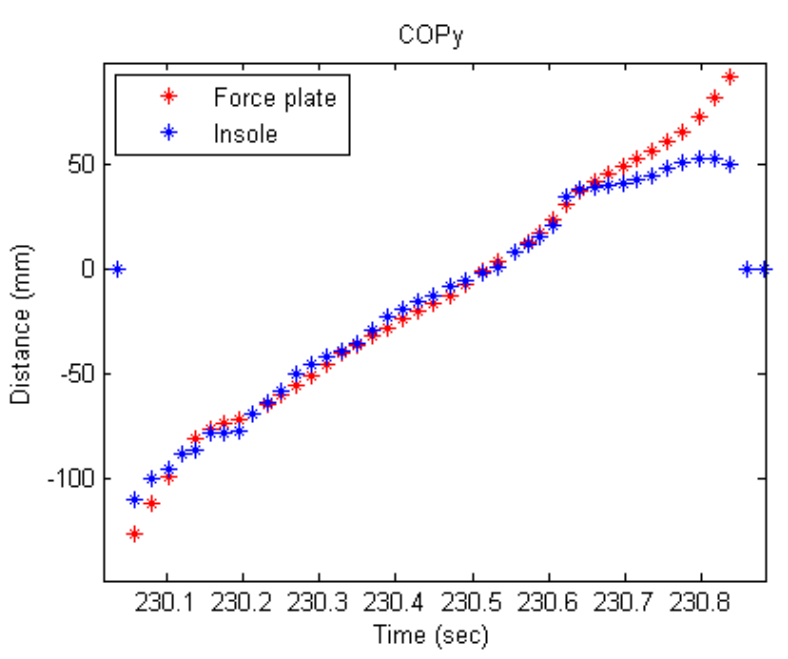

(d) $\mathrm{COPy}$

Figure D.9: Comparison of insole (blue) and force plate (red) measurements of GRF and COP for one example step of the walking experiments from subject 5 . 


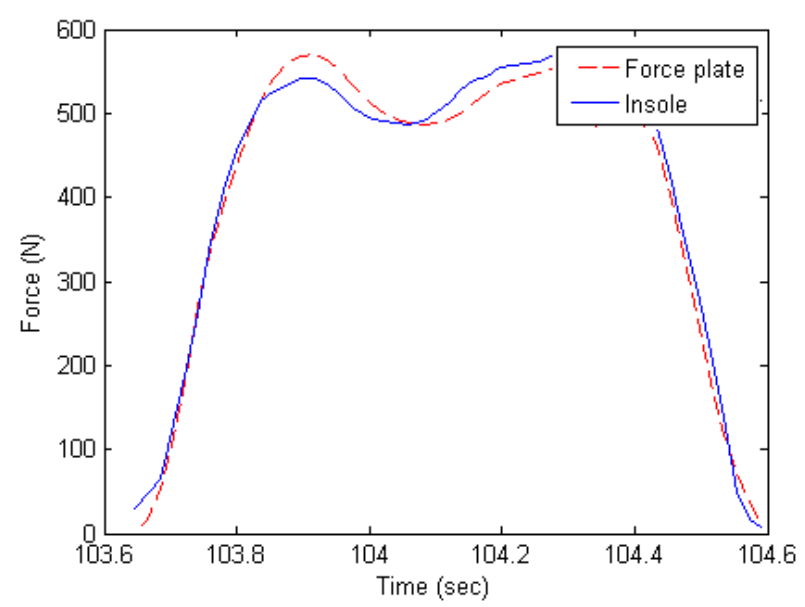

(a) VGRF

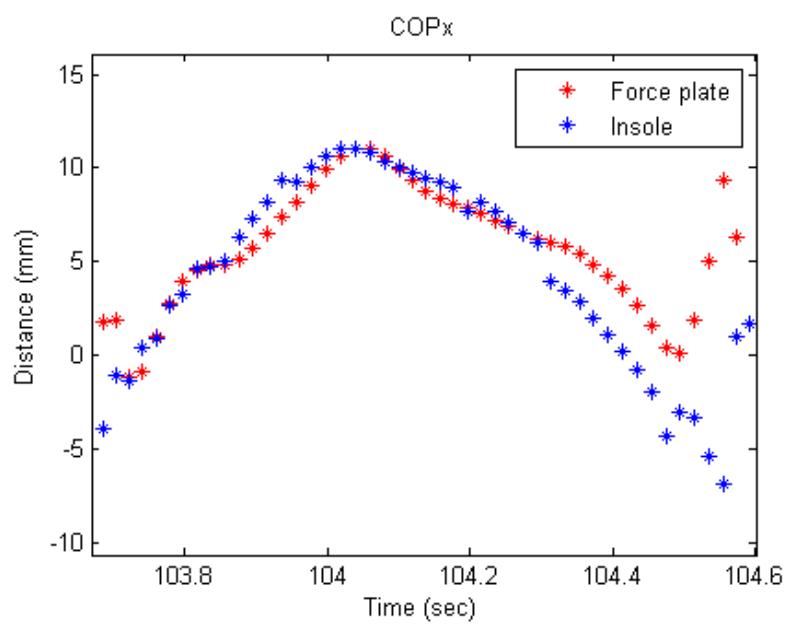

(c) $\operatorname{COPx}$

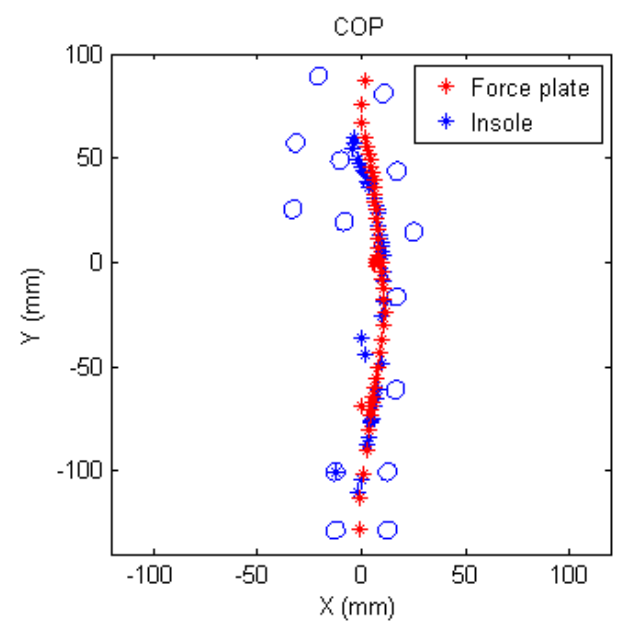

(b) $\mathrm{COP}$

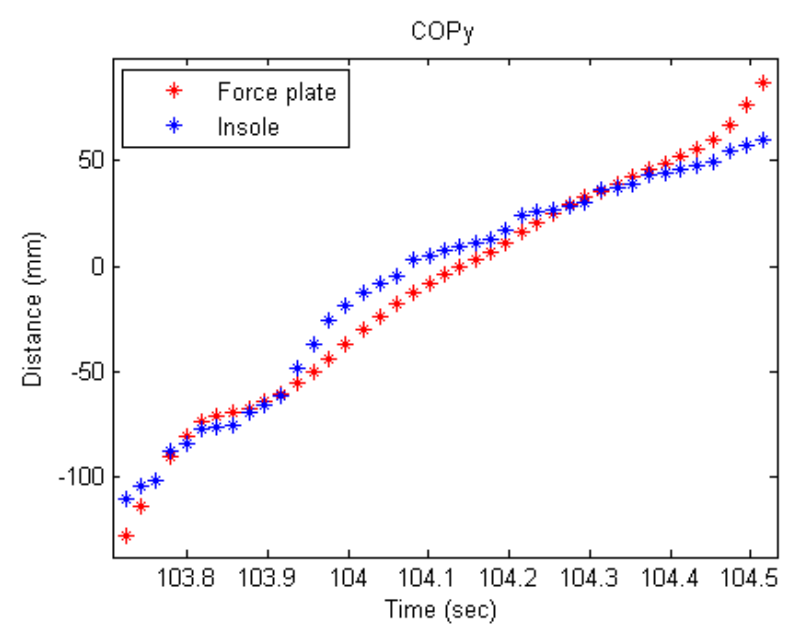

(d) $\mathrm{COPy}$

Figure D.10: Comparison of insole (blue) and force plate (red) measurements of GRF and COP for one example step of the walking experiments from subject6. 


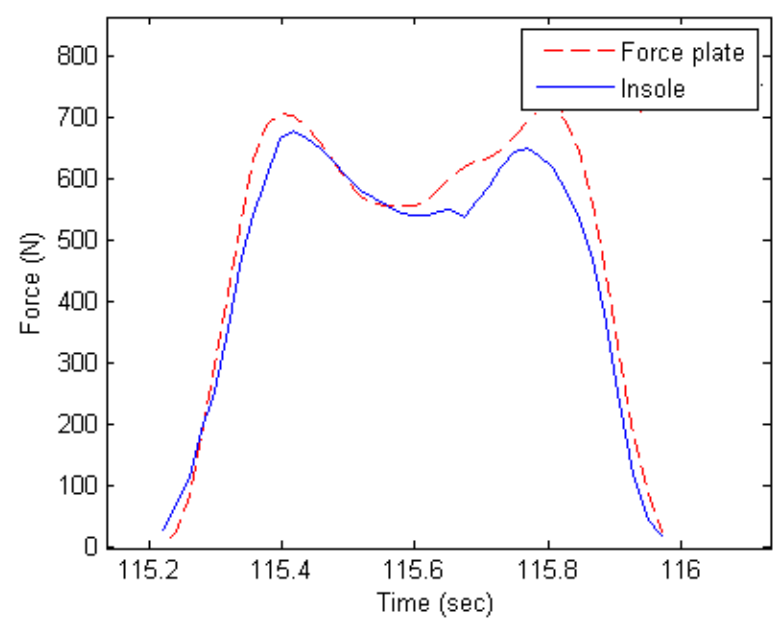

(a) VGRF

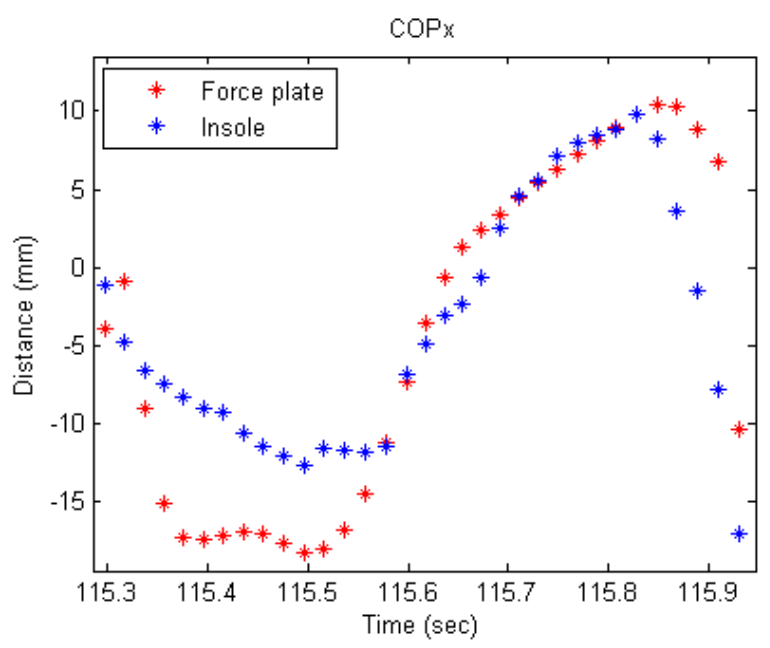

(c) $\mathrm{COPx}$

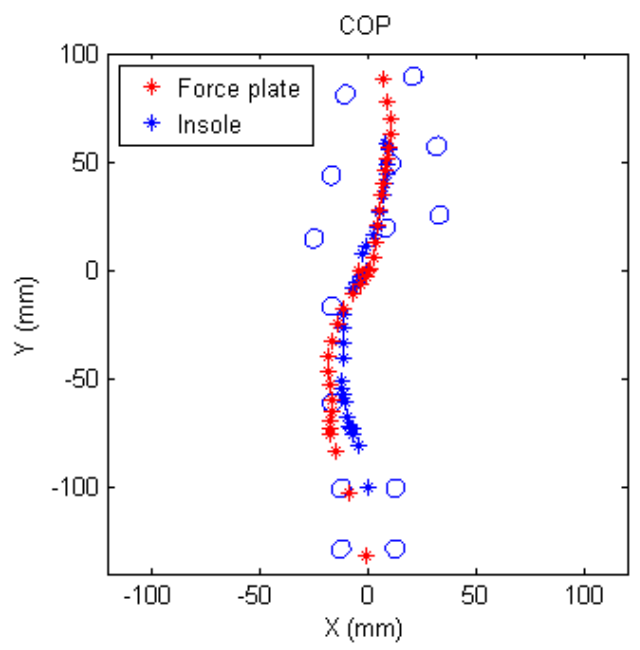

(b) COP

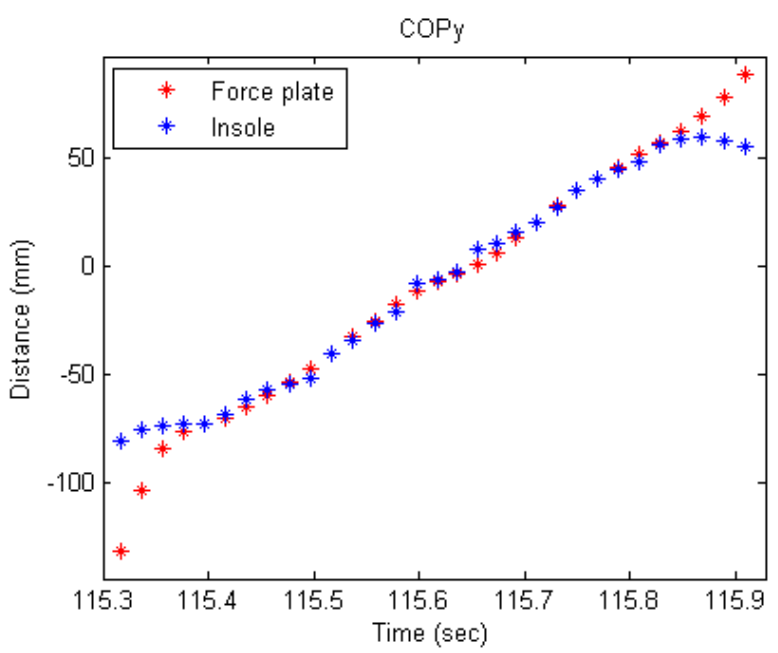

(d) COPy

Figure D.11: Comparison of insole (blue) and force plate (red) measurements of GRF and COP for one example step of the walking experiments from subject7. 